\title{
DIFFERENTIABLE DYNAMICAL SYSTEMS ${ }^{1}$
}

\author{
BY S. SMALE
}

\section{PART I. DIFFEOMORPHISMS}

\section{I.1. Introduction to conjugacy problems for diffeomorphisms. This} is a survey article on the area of global analysis defined by differentiable dynamical systems or equivalently the action (differentiable) of a Lie group $G$ on a manifold $M$. An action is a homomorphism $G \rightarrow \operatorname{Diff}(M)$ such that the induced map $G \times M \rightarrow M$ is differentiable. Here Diff $(M)$ is the group of all diffeomorphisms of $M$ and a diffeomorphism is a differentiable map with a differentiable inverse. Everything will be discussed here from the $C^{\infty}$ or $C^{r}$ point of view. All manifolds maps, etc. will be differentiable $\left(C^{r}, 1 \leqq r \leqq \infty\right)$ unless stated otherwise.

In the beginning we will be restricted to the discrete case, $G=Z$. Here $Z$ denotes the integers, $Z^{+}$the positive integers. By taking a generator $f \in \operatorname{Diff}(M)$, this amounts to studying diffeomorphisms on a manifold from the point of view of orbit structure. The orbit of $x \in M$, relative to $f$, is the subset $\left\{f^{m}(x) \mid m \in Z\right\}$ of $M$ or else the map $Z \rightarrow M$ which sends $m$ into $f^{m}(x)$. The finite orbits are called periodic orbits and their points, periodic points. Thus $x \in M$ is a periodic point if $f^{m}(x)=x$ for some $m \in Z^{+}$. Here $m$ is called a period of $x$ and if $m=1, x$ is a fixed point. Our problem is to study the global orbit structure, i.e., all of the orbits on $M$.

The main motivation for this problem comes from ordinary differential equations, which essentially corresponds to $G=R, R$ the reals acting on $M$. There are two reasons for this leading to the diffeomorphism problem. One is that certain differential equations have cross-sections (see, e.g., [114]) and in this case the qualitative study of the differential equation reduces to the study of an associated diffeomorphism of the cross-section. This is the reason why Poincare [90] and Birkhoff [19] studied diffeomorphisms of surfaces,

I believe there is a second and more important reason for studying the diffeomorphism problem (besides its great natural beauty). That is, the same phenomena and problems of the qualitative theory of ordinary differential equations are present in their simplest form in the diffeomorphism problem. Having first found theorems in the dif-

1 The preparation of this paper was supported by the National Science Foundation under grant GN-530 to the American Mathematical Society and partially supported by NSF grant GP-5798. 
feomorphism case, it is usually a secondary task to translate the results back into the differential equations framework.

Assuming $M$ compact, we put on $\operatorname{Diff}(M)$ the topology of uniform $C^{r}$ convergence. We will usually keep $M$ compact because for noncompact $M$, there are different behaviours at infinity that one could consider. See, for example, [86]. These lead to different problems and we don't wish to get into such questions here.

One of the first things that one observes is the need to exclude degenerate elements of $\operatorname{Diff}(M)$. For example, given any nonempty closed set $F \subset M$, there is $f \in \operatorname{Diff}(M)$ such that the fixed point set $\operatorname{Fix}(f)=F$. For a number of reasons, if $F$ is not discrete we would like to exclude such $f$. The set of $f \in \operatorname{Diff}(M)$ such that $\operatorname{Fix}(f)$ is discrete (or finite, since we assume $M$ compact) contains an open dense set of $\operatorname{Diff}(M)$. This leads to the notion of generic properties of diffeomorphisms. A Baire set of a complete metrizable space is the intersection of a countable number of open dense sets. Then a generic property is a property that is true for diffeomorphisms belonging to some Baire set of $\operatorname{Diff}(M)$. We will never speak of generic $f \in \operatorname{Diff}(M)$ (this is usually taken to mean that $f$ has a lot of generic properties!). Thus "Fix $(f)$ is finite" is a generic property and a little more since open dense is stronger than Baire (see $\$ I .6$ for more details and references).

It is important in proceeding to consider formal equivalence relations on $\operatorname{Diff}(M)$ which will preserve the orbit structure in some sense. Furthermore associated to each equivalence relation there is a notion of stability. More precisely if the equivalence relation on $\operatorname{Diff}(M)$ is called $E, f \in \operatorname{Diff}(M)$ is called E-stable if there is a neighborhood $N(f)$ of $f$ in $\operatorname{Diff}(M)$ such that if $f^{\prime} \in N(f)$ (or $f^{\prime}$ approximates $f$ sufficiently), then $f$ and $f^{\prime}$ are in the same $E$ equivalence class.

It would give a reasonable picture (see [111], [112]) to have a dense open set $U \subset \operatorname{Diff}(M)$ such that our equivalence classes could be distinguished by numerical and algebraic invariants. This is, in fact, our goal. If this is to be the case, the desired equivalence $E$ on $\operatorname{Diff}(M)$ should have the property that the $E$-stable diffeomorphisms are dense in Diff $(M)$. With this background we look at some particular equivalence relations.

The notion of conjugacy first comes to mind. Say $f, f^{\prime} \in \operatorname{Diff}(M)$ are differentiably (or topologically) conjugate if there is a diffeomorphism (or homeomorphism) $h: M \rightarrow M$ such that $h f=f^{\prime} h$. Differentiable conjugacy is too fine in view of the above considerations. This is due to the fact that the eigenvalues of the derivative at a fixed point are differentiable conjugacy invariants. The notion of stability 
associated to topological conjugacy is called structural stability, and for some time it was thought that structurally stable diffeomorphisms might be dense in Diff $(M)$. This turned out to be false [116]. Thus by our earlier consideration we should relax our relation on $\operatorname{Diff}(M)$ of topological conjugacy. Before doing this we introduce some basic ideas about G. D. Birkhoff's nonwandering points [15].

If $f \in \operatorname{Diff}(M), x \in M$ is called a wandering point when there is a neighborhood $U$ of $x$ such that $\bigcup_{|m|>0} f^{m}(U) \cap U=\varnothing$. The wandering points clearly form an invariant open subset of $M$. A point will be called nonwandering if it is not a wandering point. These nonwandering points are those with the mildest possible form of recurrence. They form a closed invariant set which we will always refer to as $\Omega=\Omega(f)$.

We propose now the equivalence "topological conjugacy on $\Omega$." That is $f, f^{\prime} \in \operatorname{Diff}(M)$ are topologically conjugate on $\Omega$ if there is a homeomorphism $h: \Omega(f) \rightarrow \Omega\left(f^{\prime}\right)$ such that $h f=f^{\prime} h$. The corresponding stability will be called simply $\Omega$-stability. So $f \in \operatorname{Diff}(M)$ will be called $\Omega$-stable if sufficiently good approximations $f^{\prime}$ are topologically conjugate on $\Omega$.

In general one can speak of topological conjugacy for homeomorphisms and even two homeomorphisms of different topological spaces, $f: X \rightarrow X, f^{\prime}: X^{\prime} \rightarrow X^{\prime}$. Then the conjugacy $h$ is a homeomorphism $h: X \rightarrow X^{\prime}$.

We end $\S I .1$ by giving some notations and conventions we follow.

Anytime the topology on $\operatorname{Diff}(M)$ is involved $M$ will be assumed compact.

Simply connected $X$ means $\Pi_{1}(X)$ and $\Pi_{0}(X)$ are trivial. We suppose that our manifolds are always connected.

Dim $M$ means the dimension of $M$.

The tangent bundle of a manifold will be denoted by $T(M)$, the tangent space at $x \in M$ by $T_{x}(M)$. The derivative of $f: M \rightarrow M$ will be denoted by $D f$ and considered as a bundle map $D f: T(M) \rightarrow T(M)$. At a point $x \in M$, it becomes $D f(x): T_{x}(M) \rightarrow T_{f(x)}(M)$. An immersion is a differentiable map such that the derivative at each point is injective.

A closed invariant set $\Lambda$ of $f \in \operatorname{Diff}(M)$ will be called indecomposable if $\Lambda$ cannot be written $\Lambda=\Lambda_{1} \cup \Lambda_{2}, \Lambda_{1}, \Lambda_{2}$ nonempty disjoint closed invariant subsets.

Finally if $\lambda$ is an eigenvalue of a linear transformation $u: V \rightarrow V$, we will define its eigenspace $E_{\lambda}=\left\{x \in V \mid(u-\lambda I)^{m}(x)=0\right.$, some $\left.m \in Z^{+}\right\}$. Then $\lambda$ will be counted with multiplicity $\operatorname{dim} E_{\lambda}$.

Two earlier surveys on this subject are [85] and [112]. 
Part $I$ is the heart of the paper, including a number of new ideas, and is devoted to problems spoken of in this section. Part II briefly extends the results to the ordinary differential equation case $(G=R)$ and Part III discusses other aspects of the differential equation problem. Part IV is devoted to possibilities for more general Lie groups $G$.

I would like to acknowledge here many very helpful discussions with other mathematicians. This includes especially D. Anosov, A. Borel, A. Haefliger, M. Hirsch, N. Kopell, I. Kupka, J. Moser, R. Narasimhan, J. Palis, M. Peixoto, C. Pugh, M. Shub and R. Thom'

I.2. The simplest examples. This section is devoted to giving a description of a class of $\Omega$-stable diffeomorphisms which are the simplest as far as the orbit structure goes. To develop or even define these diffeomorphisms, we will need the basic idea of a stable manifold.

A linear automorphism $u$ of a (say real) finite dimensional vector space $V, u: V \rightarrow V$ will be called hyperbolic if its eigenvalues $\lambda_{i}$ satisfy $\left|\lambda_{i}\right| \neq 1$ all $i$. We emphasize that complex eigenvalues are permitted. The automorphism $u$ will be called contracting if $\left|\lambda_{i}\right|<1$ for all $i$, expanding if $\left|\lambda_{i}\right|>1$ for all $i$, and of saddle type otherwise. Thus the inverse of an expanding automorphism is a contracting automorphism and vice versa.

Observe that for hyperbolic $u: V \rightarrow V$ we have a canonical, invariant (under $u$ ) splitting of $V, V=V^{s}+V^{u}$ (direct sum) where $V^{s}$ is the eigenspace of $u$ corresponding to eigenvalues less than 1 in absolute value and $V^{u}$ the eigenspace of the remaining eigenvalues. Thus $u$ restricted to $V^{s}$ is contracting and $u$ restricted to $V^{u}$ is expanding. This gives rise to the following familiar picture for such $u$.

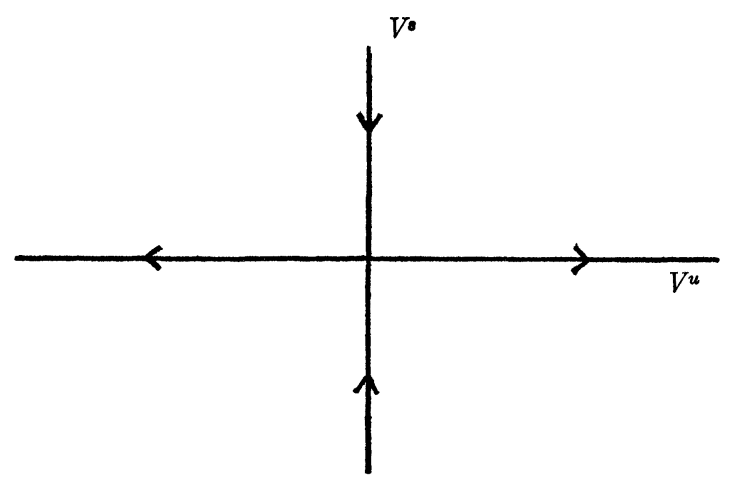

Figure 1 
Note that the hyperbolic elements of the general linear group GL( $V)$ are open and dense.

Now suppose $f: M \rightarrow M$ is a diffeomorphism with a fixed point $p \in M$ (a local diffeomorphism $f: U \rightarrow M, U$ an open subset of $M$, $p \in U, f(p)=p$ would be sufficient for some of the following discussion). The derivative of $f$ at $p, D f(p)$, may be considered to be a linear automorphism of the tangent space of $M$ at $p$, i.e., $D f(p): T_{p}(M)$ $\rightarrow T_{p}(M)$. We will say that $p$ is a hyperbolic fixed point of $f$, or simply a hyperbolic fixed point, if $D f(p)$ is hyperbolic in the sense of the previous paragraphs.

We will call a periodic point $p$ of period $m \in Z^{+}$of $f: M \rightarrow M$ hyperbolic if it is a hyperbolic fixed point of $f^{m}$. Similarly, $p$ is a contracting or expanding periodic point if $D f^{m}(p)$ is a contracting (or expanding) linear automorphism.

A (global) contraction of a differentiable manifold $V$ is a diffeomorphism $g: V \rightarrow V$ which is topologically conjugate to a linear contraction (i.e., a linear contracting automorphism) $u: V^{\prime} \rightarrow V^{\prime}$. Of course a contraction will have a unique fixed point.

For hyperbolic fixed points we have stable manifolds defined according to the following theorem.

(2.1) Stable Manifold Theorem. Suppose $p \in M$ is a hyperbolic fixed point of a diffeomorphism $f: M \rightarrow M$ with $T_{p}(M)=V^{s}+V^{u}$ the corresponding decomposition under $D f(p)$. Then there exists a contraction $g: W^{s}(p) \rightarrow W^{s}(p)$ with fixed point $p_{0}$ and an injective equivariant immersion $J: W^{s}(p) \rightarrow M$ such that $J\left(p_{0}\right)=p$ and $D J\left(p_{0}\right): T_{p_{0}}\left(W^{s}(p)\right)$ $\rightarrow T_{p}(M)$ is an isomorphism onto $V^{s} \subset T_{p}(M)$. Furthermore the image $J\left(W^{s}(p)\right)$ may be characterized as the set of $x \in M$ with the property $f^{m}(x) \rightarrow p$ as $m \rightarrow \infty$.

Equivariance here means simply that $J g=f J$. Note that the derivative condition implies that the dimensions of $V^{s}$ and $W^{s}(p)$ are the same.

The image of $J$ is invariant under $f$, and frequently we will identify points under $J$ so that $W^{s}(p) \subset M$. In general, $J$ will not be a homeomorphism onto its image (see the toral example of $\S 1.3$ ), so that the original $W^{s}(p)$ and $W^{s}(p)$ is a subset of $M$ have different topologies and this is the only way they differ. Both are called the stable manifold of $f$ at $p$. When it is important to specify the topology, we will say intrinsic for the original topology and the manifold topology for the other topology on $W^{s}(p)$.

For analytic diffeomorphisms of two dimensional manifolds, this theorem was known to Poincaré [90] and used by Birkhoff [16]. 
The proof of (2.1) starts by showing the existence of a "local stable manifold," $W^{s_{100}}(p)$. This is due to Perron [88]. He uses iteration methods in a function space to solve a functional equation for $J$ in a neighborhood of $p$. Further references to versions of this theorem are [2], [24], [39], [120] (most often these papers concern themselves with the differential equations analogue, so one has to make a translation of the results). The global theorem, (2.1), follows easily from the local theorem by so to speak "topological continuation." One takes for $W^{s}(p)$ the subset $\cup_{m \in Z^{+}} f^{-m} W^{s}$ loc $(p)$ of $M$. See [114] for more details.

For a hyperbolic fixed point $p$ of a diffeomorphism $f: M \rightarrow M$, the unstable manifold $W^{u}(p)$ is defined as the stable manifold of $f^{-1}$ at $p$. Thus $W^{u}(p)$ passes through $p$ and is tangent to $V^{u}$ in the notation of (2.1).

For a periodic point $q$ of $f \in \operatorname{Diff}(M), f^{m}(q)=q, m \in Z^{+}$, one defines the stable and unstable manifolds, $W^{s}(p), W^{u}(q)$ as the stable and unstable manifolds for $q$ as a fixed point of $f^{m}$.

Although each $W^{s}(p)$ is a 1-1 immersion, there is no reason why $W^{s}(p)$ and $W^{u}(q)$ cannot intersect each other. In fact as the toral example of $\S I .3$ shows, it may happen that $W^{s}(p)$ intersects $W^{u}(p)$ (this is called a homoclinic point; see $\S I .5$ ).

We now are in a position to describe the examples, or the class of examples, we mentioned earlier. As a prototype it is worthwhile to keep in mind the diffeomorphism $g_{0}: S^{2} \rightarrow S^{2}$ of the 2-sphere which can be described complex analytically on the Riemann sphere by $z \rightarrow 2 z$. The two fixed points are 0 which is expanding and $\infty$, contracting. Then $W^{u}(0)=S^{2}-\infty, W^{s}(0)=0, W^{u}(\infty)=\infty, W^{s}(\infty)=S^{2}-0$. It is easily checked that $g_{0}$ is structurally stable. Of course one may construct a similar example on $S^{n}$ with two fixed points.

More generally we will consider $f \in \operatorname{Diff}(M), M$ compact, which satisfies the following three conditions:

(2.2) (1) $\Omega$, the nonwandering set, is finite.

(2) The periodic points of $f$ are hyperbolic.

(3) (Transversal intersection condition) For each $p, q \in \Omega, W^{s}(p)$ and $W^{u}(q)$ have transversal intersection.

It follows from (1) that $\Omega$ consists of periodic points and (2) that $W^{s}(p), W^{u}(q)$ are defined for $p, q \in \Omega$. The last condition means that whenever $x \in W^{s}(p) \cap W^{u}(q)$, then $T_{x}\left(W^{s}(p)\right)$ and $T_{x}\left(W^{u}(q)\right)$ span $T_{x}(M)$.

It is trivial to check that the above $g_{0}: S^{2} \rightarrow S^{2}$ satisfies (1)-(3).

Furthermore, consider for the moment, diffeomorphisms of the circle $S^{1}$ satisfying (2.2). In this case (2.2)-(3) is vacuously satisfied, 
and it is easily checked directly that these diffeomorphisms are open. By perturbing an arbitrary $f \in \operatorname{Diff}\left(S^{1}\right)$ so that its rotation number [24] becomes rational and a further approximation to obtain (2.2)-(1) we obtain the fact that these diffeomorphisms are open and dense in $\operatorname{Diff}\left(S^{1}\right)$ (Peixoto's theorem [84]). As one goes around the circle, the expanding and contracting periodic points alternate. The structural stability in the case is easy to check [84].

If $A \subset B, \operatorname{clos} A$ denotes the closure of $A$ in $B$.

(2.3) Theorem [109]. Suppose $f: M \rightarrow M$ satisfies (2.2). Then (a) for each $p \in \Omega, W^{s}(p)$ is imbedded in $M$ and $M=\cup_{p \in \Omega} W^{s}(p)$ (disjoint union of course).

(b) clos $W^{s}(p)$ is the union of $W^{s}(q)$, for $q$ in some subset of $\Omega$. If we write $\gamma \leqq \gamma^{\prime}$ for periodic orbits $\gamma, \gamma^{\prime}$ whenever $U_{p \in \gamma} W^{s}(p)$ C clos $U_{q \in \gamma^{\prime}} W^{s}(q)$, then $\leqq$ is a partial ordering. If $\gamma \leqq \gamma^{\prime}$ and $p \in \gamma$, $q \in \gamma^{\prime}$, then $\operatorname{dim} W^{s}(p) \leqq \operatorname{dim} W^{s}(q)$.

(c) One has the following Morse inequalities:

$$
\begin{aligned}
M_{0} & >B_{0}, \\
M_{1}-M_{0} & \geqq B_{1}-B_{0}, \\
& \vdots \\
\sum_{i=0}^{\operatorname{dim} M}(-1)^{i} M_{i} & =\sum_{i=0}^{\operatorname{dim} M}(-1)^{i} B_{i} .
\end{aligned}
$$

Here $B_{i}$ is the $i$ th betti number of $M$ and $M_{j}$ is the number of periodic points $p$ such that $\operatorname{dim} W^{s}(p)=j$.

The essence of the proof of (2.3) is in a more general context in $\$ I .8$.

Using (2.3) (b), one may "represent" a diffeomorphism satisfying (2.2) by a diagram where the vertices of the diagram correspond to periodic orbits and oriented segments are placed between orbits $\gamma$ and $\gamma^{\prime}$ when $\gamma \leqq \gamma^{\prime}$ but there is no other $\gamma^{\prime \prime}$ such that $\gamma \leqq \gamma^{\prime \prime} \leqq \gamma^{\prime}$.

A labeled diagram is a diagram with the following additional data attached to each vertex $\gamma$. The additional data is the germ of the topological conjugacy class of $f^{m}$ at $x \in \gamma$ where $m$ is the least period of $\gamma$. This germ is described precisely by the dimensions of $W^{s}(x), W^{u}(x)$ and whether $f: W^{u}(x) \rightarrow W^{u}(x), f: W^{s}(x) \rightarrow W^{s}(x)$ are orientation preserving or reversing (this is a consequence of the theorem of Hartman [39] and Grobman (see [74] which says that locally a diffeomorphism at a hyperbolic fixed point is topologically equivalent to its derivative at that point).

(2.4) Problem. (a) Exactly what (abstract) labeled diagrams occur as diagrams of diffeomorphisms satisfying (2.2)? 
(b) Given compact $M$ exactly what (abstract) labeled diagrams occur as the labeled diagrams of diffeomorphisms of $M$ satisfying (2.2)?

Note that (2.3) (c) may be viewed as a restriction on the kind of diagrams that can occur.

Figure 2 below gives the phase portrait or orbit structure of an example of a diffeomorphism of the 2-sphere satisfying (2.2).

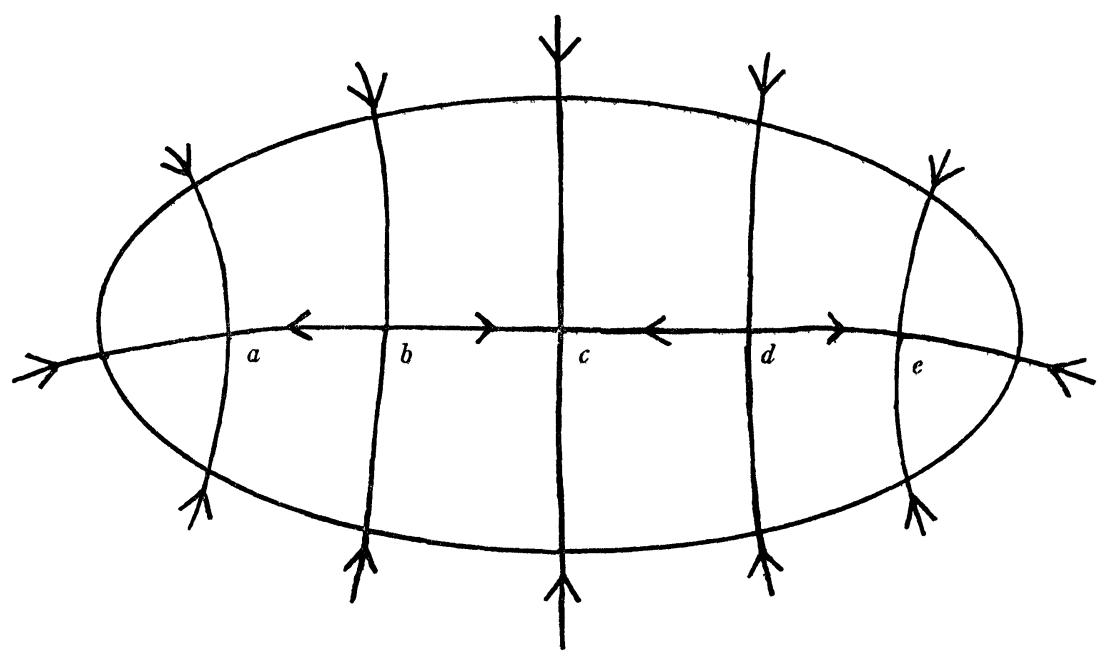

Figure 2

Here the main disk is to be contracting into itself with one expanding fixed point $p$ outside. Inside the disk are five fixed points $a, c, e$ all contracting and $b, d$ of saddle type. The diagram for this diffeomorphism is given by

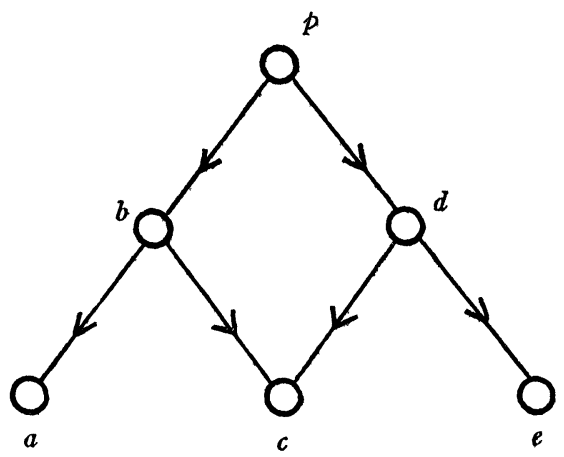

Figure 3 
Among other interesting results on this subject, Jacob Palis [82] shows that diffeomorphisms satisfying (2.2) form an open set in $\operatorname{Diff}(M)$. He also shows that the diagram of the perturbation of $f$ is naturally "isomorphic" to the diagram of $f$.

Even though the above facts give something of a "phase portrait" (in the terminology of [57]), a number of problems on this subject still remain. For example

(2.5) Problem [109], [111]. Are these diffeomorphisms (of 2.2) structurally stable? J. Palis has given an affirmative answer in dimension 2.

(2.6) What homotopy classes of continuous maps (homotopy equivalences) admit diffeomorphisms of (2.2) type? A necessary condition which follows from the Lefschetz trace formula is that $\left|\Lambda\left(f^{m}\right)\right|$ $<C$, where $\Lambda$ is the Lefschetz number and $C$ is a constant independent of $m$.

The gradient-like diffeomorphisms are a special class of diffeomorphisms satisfying (2.2), the most transparent and easily understood. More precisely a gradient-like diffeomorphism is one which satisfies (2.2) and has the additional property that if $W^{s}(p) \leqq W^{s}(q)$, then $\operatorname{dim} W^{s}(p)$ is actually less than $\operatorname{dim} W^{s}(q)$. For example the diffeomorphism of diagram 2 is gradient-like.

More generally every gradient flow with mild transversality and nondegeneracy conditions (see [110]) generates a gradient-like diffeomorphism. This construction guarantees the existence of gradientlike diffeomorphisms (satisfying (2.2)) on every compact manifold. In this way the above Morse inequalities (2.3) (c) include the usual ones. Even for these diffeomorphisms, structural stability is not yet proved.

For a 2-dimensional diffeomorphism satisfying (2.2) a heteroclinic point is a point $x \in W^{u}(p) \cap W^{s}(q)$ where $\operatorname{dim} W^{s}(q)=\operatorname{dim} W^{s}(p)$ $=1$, so that at $x, T_{x}\left(W^{u}(p)\right)$ and $T_{x}\left(W^{s}(q)\right)$ intersect in just one point in $T_{x}(M)$. Clearly a diffeomorphism possessing a heteroclinic point is not gradient-like. The orbit of a heteroclinic point consists of other heteroclinic points.

The interested reader will be able to check that the existence of the heteroclinic point $x$ above forces $W^{s}(q)$ to oscillate strongly as it gets close to $p$ and $W^{s}(p)$. The boundary of $W^{s}(q)$ contains $W^{s}(p)$. The picture looks something like Figure 4.

To obtain a global example one may modify the diffeomorphism of the 2-sphere of Figure 2. The result will be something like Figure 5. 

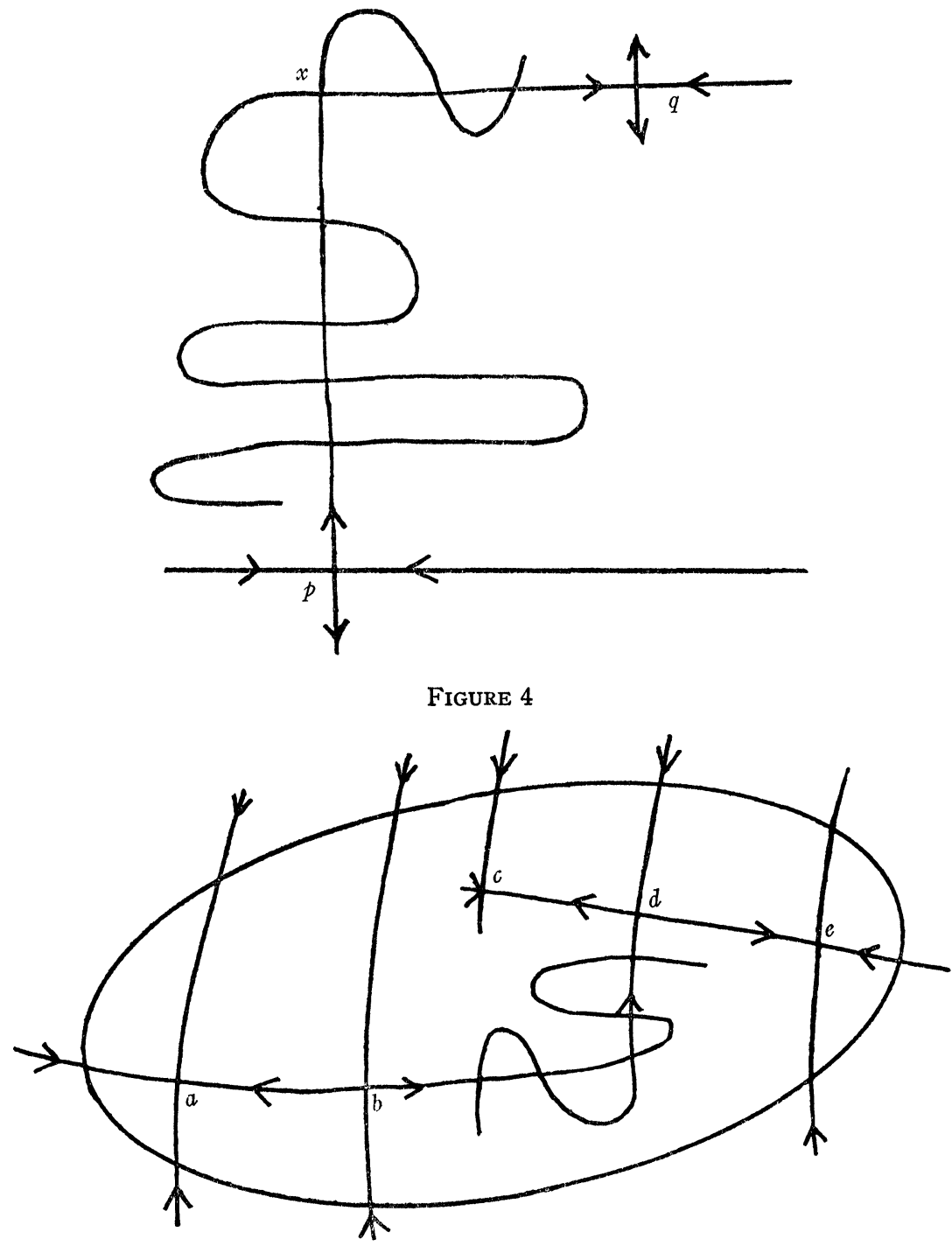

Figure 5

Its diagram is given in Figure 6.

We discuss relaxing or dropping some of the conditions (1), (2), (3) of (2.2). The rest of Part I is concerned with weakening (1), so we consider now (2) and (3). It seems to us that dropping (2) or even modifying (2) significantly would take one far from the picture described by (2.3). What happens if (3) is relaxed?

Consider the following substitute for (3). 


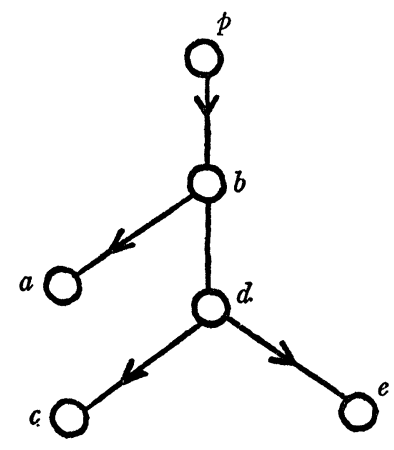

Figure 6

$\left(3^{\prime}\right)$ If $W^{s}(p)$ and $W^{u}(q)$ intersect at all, then there is a point of transversal intersection of $W^{s}(p)$ and $W^{u}(q)$.

With the weaker $\left(3^{\prime}\right)$ replacing (3) one still is able to prove (2.3). Moreover with either $(3)$ or $\left(3^{\prime}\right)$, the relation $\leqq$ and the diagram are invariant under perturbation. However, with a weakened version of (2.2) there is no hope of proving structural stability as the simplest counter-examples show. In fact for structurally stable $f \in \operatorname{Diff}(M)$, (3) is satisfied.

The bulk of this section is taken from [109] with some updating, a few examples and other points added. On the other hand, many of the ideas go back quite a number of years. Certainly the local theory as mentioned in the text is of this character. Also Poincaré [89], Birkhoff [16], and M. Morse [68] all had some parts of this global picture. Since this earlier work, Andronov and Pontrjagin [6], Elsgolts [30], Peixoto [83], Reeb [94], and Thom [124] among others had made contributions toward the picture given in this section.

Besides giving simple examples of $\Omega$-stable diffeomorphisms, the material in this section serves as an introduction to the more general theory of $\S I .6$, where a number of these concepts have natural extensions.

I.3. Anosov diffeomorphisms. The examples of this section (at least roughly speaking) are at the opposite extreme from those of the preceding section in that the whole manifold consists of nonwandering points and the periodic points are dense. This is in contrast to $\Omega$ being finite as in $\S 1.2$. We give first the simplest examples of Anosov diffeomorphisms, the toral diffeomorphisms.

Consider $f_{0}$, a $2 \times 2$ matrix with integer entries and determinant \pm 1 , i.e., $f_{0} \in \mathrm{GL}(2, Z)$. Then $f_{0}$ can be thought of as a linear transformation of the plane $R^{2}$ which preserves the lattice $L$ of points with integer coordinates. There is an induced diffeomorphism $f$ of the quo- 
tient $R^{2} / L=T^{2}, 2$-dimensional torus, on to itself. This diffeomorphism $f: T^{2} \rightarrow T^{2}$ has a fixed point $p$ corresponding to the origin of $R^{2}$.

Now suppose $f_{0}$ is hyperbolic, for example

$$
\left(\begin{array}{ll}
1 & 2 \\
1 & 1
\end{array}\right) .
$$

Then $p$ will be a hyperbolic fixed point of $f$ and the stable and unstable manifolds $W^{s}(p)$ and $W^{u}(p)$ will be the image of the eigenspaces of $f_{0}$ under the projection $\Pi: R^{2} \rightarrow T^{2}$ (since $f_{0}$ is hyperbolic, the eigenvalues $\lambda, \mu$ are real and satisfy $|\lambda|>1>|\mu|>0$ with 1-dimensional eigenspaces). Since $W^{s}(p)$ is a 1-1 immersion, it winds densely around the torus and similarly with $W^{u}(p)$.

The intersection points, in $W^{s}(p) \cap W^{u}(p)$ (called homoclinic points, see $\$ I .5)$, are clearly dense in $T^{2}$, and it can also be shown that the periodic points of $f$ are dense in $T^{2}$. This follows from an algebraic argument or one can use the generalized Birkhoff theorem (see I.(5.6)).

For any periodic point $q \in T^{2}$ of period $m$, the derivative of $f^{m}$ at $q$ can be thought of as $f_{0}^{m}: R^{2} \rightarrow R^{2}$ after identifying $T_{q}\left(T^{2}\right)$ and $R^{2}$ by translation. The stable manifold $W^{s}(q)$ will then just be the translate of $W^{s}(p)$. From the Lefschetz Trace Formula (see $\$ I .4$ ), the number of periodic points $N_{m}$ of period $m$ is $1-\left(\lambda^{m}+\mu^{m}\right)+$ degree $f$. Then any $g$ in the same homotopy class as $f$ must have an infinite number of periodic points and therefore cannot satisfy (2.2). It turns out that $f$ is structurally stable so that any perturbation of $f^{\prime}$ will also have periodic points dense in $T^{2}$. Everything said about $f_{0}$ extends to hyperbolic $f_{0} \in \mathrm{GL}(n, Z)$, defining what we will call toral diffeomorphisms.

The definitive version of the structural stability of $f$ is contained in the work of Anosov [7], [8] which we will describe now.

We recall that a Riemannian vector space bundle $E$ over a space $X$ is a vector space bundle such that each fiber $E_{x}$ is equipped with an inner product $(,)_{x}$ in a continuous manner. This allows one to speak of the norm $\|v\|$ of a vector $v \in E_{x}$. A bundle map between vector space bundles is a fiber preserving $\phi: E \rightarrow E$ of a Riemannian vector space bundle into itself and will be called contracting if there exists $C>0,0<\lambda<1$ such that for all $v \in E, m \in Z^{+}$

$$
\left\|\phi^{m}(v)\right\|<C \lambda^{m}\|v\| \text {. }
$$

It will be called expanding if there exists $d>0, \mu>1$ such that for all $v \in E, m \in Z^{+}$

$$
\left\|\phi^{m}(v)\right\|>d \mu^{m}\|v\|
$$


Above, we really are just using the norm in each fiber, not the inner product.

(3.1) Proposition. If $X$ is compact, then the property of being contracting or expanding for $\phi: E \rightarrow E, E$ a Riemannian vector space bundle over $X$ is independent of the Riemannian metric.

Proof. Two norms \|\|$,\|,\|^{\prime}$ on fibers of $E$ are related locally, and hence globally by $a\|\| \leqq\|\|^{\prime} \leqq b\|\|$ for some $a, b>0$. Thus if $\left\|\phi^{m}(v)\right\| \leqq c \lambda^{m}\|v\|$, then $\left\|\phi^{m}(v)\right\|^{\prime} \leqq c(b / a) \lambda^{m}\|v\|^{\prime}$.

(3.2) Proposition. The inverse of a contracting bundle automorphism is an expanding bundle automorphism and vice-versa.

Proof. Suppose $\left\|\phi^{m}(v)\right\| \leqq c \lambda^{m}\|v\|$. Then writing $\phi^{m}(v)=w$, $\left\|\phi^{-m}(w)\right\| \geqq(1 / c)(1 / \lambda)^{m}\|w\|$.

Actually, J. Mather has shown me how to renorm the bundle so as to make $c=1$ in the defining condition for contracting bundle automorphisms.

Since on every vector space bundle there exist Riemannian metrics, by (3.1) we can dispense with the Riemannian structure when speaking of contracting bundle maps of bundles over compact spaces.

If $f: M \rightarrow M$ is a diffeomorphism, then the derivative $D f: T(M)$ $\rightarrow T(M)$ is a bundle automorphism of the tangent bundle of $M$.

Suppose now that $M$ is Riemannian so that $T(M)$ is a Riemannian vector space bundle over $M$. We will say that $f: M \rightarrow M$ is an Anosov diffeomorphism and that $M$ has a hyperbolic structure for $f$ if the following condition is satisfied: there is a splitting of the tangent bundle $T(M)$ into a continuous (not necessarily $C^{r}$ ) Whitney sum $T(M)$ $=E^{s}+E^{u}$, invariant under $D f: T(M) \rightarrow T(M)$ so that $D f: E^{s}+E^{s}$ is contracting and $D f: E^{u} \rightarrow E^{u}$ is expanding.

The Riemannian structure of $T(M)$ restricts to give a Riemannian structure on $E^{s}$ and $E^{u}$ so that this condition makes sense; furthermore, in case $M$ is compact, by our previous comment, the Riemannian structure is unnecessary.

(3.3) Theorem (Anosov) ([7], [8]). An Anosov diffeomorphism $f$ of a compact manifold $M$ is structurally stable. Furthermore if there is a Lebesgue invariant measure for $f$ on $M$, then the periodic points are dense and $f$ is ergodic. Finally the Anosov diffeomorphisms are an open set in $\operatorname{Diff}(M)$.

For the proof of the first statement of (3.3) see the exposition of $\mathrm{J}$. Moser's proof by J. Mather in the appendix. For the last sentence, see $\$ I .8$. 
It is apparent that the toral diffeomorphisms are Anosov diffeomorphisms; the splitting by $f$ at $p$ translates to each point of $T^{n}$ to give the desired global splitting.

From an invariant measure for a diffeomorphism $f$ of a compact manifold $M$, one can see easily that every point is nonwandering, i.e., $\Omega=M$. It is from this fact that Anosov concludes the density of the periodic points of $f$.

(3.4) Problem. Is it true that for every Anosov diffeomorphism of a compact manifold $M, \Omega=M$, or equivalently, the periodic points are dense in $M$ ? A second question is: does every Anosov diffeomorphism have a fixed point?

Motivation for this work of Anosov comes not only from the toral diffeomorphisms, but more importantly from geodesic flows on manifolds with negative curvature, where Anosov's ergodicity solves an old problem. This is the 1-parameter analogue of (3.3) and will be discussed later in our survey.

For (3.3), the basic idea of Anosov's proof is to construct through each point $p$ of $M$, a generalized stable manifold $W^{s}(p)$. This will be a 1-1 immersed cell with the property that for each $x \in W^{s}(p)$, the tangent space $T_{x}\left(W^{s}(p)\right)$ coincides with $E_{x}^{s} \subset T_{x}(M)$. Furthermore $f\left(W^{s}(p)\right)=W^{s}(f(p))$, and $x, y$ are in the same $W^{s}(p)$ if and only if $d\left(f^{m} x, f^{m} y\right) \rightarrow 0$ as $m \rightarrow \infty$.

Although each $W^{s}(p)$ is smooth, $W^{s}(p)$ only depends continuously on $p$ (recall that the splitting of $T(M)$ was only required to be continuous). One may think of the $W^{s}(p)$ giving a continuous foliation of $M$. The existence and basic properties of $W^{s}(p)$ are based on old work by Perron [88].

Theorem (3.3) states that the Anosov diffeomorphisms are an open set in Diff $(M)$. On the other hand Anosov has examples to show that this would be false if one imposed a smooth splitting of $T(M)$ rather than a continuous one in the definitions.

The following is a basic and beautiful unsolved problem.

(3.5) Problem. Find all examples of Anosov diffeomorphisms of compact manifolds (up to topological conjugacy of course) such that $M=\Omega$. What compact $M$ admit Anosov diffeomorphisms? Must $M$ be covered by Euclidean space?

There do exist nontoral Anosov diffeomorphisms. We will show this now and in fact give the most general known way of constructing Anosov diffeomorphisms.

Suppose that $G$ is a connected simply connected Lie group with Lie algebra $(5)$ and a uniform discrete subgroup $\Gamma$ (uniform means that the coset space $G / \Gamma$ is compact). Suppose also that $f_{0}: G \rightarrow G$ is a continuous automorphism such that $f_{0}(\Gamma)=\Gamma$ and the derivative at 
the identity $f_{0}^{\prime}: T_{e}(G) \rightarrow T_{e}(G)$ is hyperbolic (throughout this discussion it will be helpful to keep the toral case, with $G=R^{n}$, in mind). If $T_{e}(G)$ is identified with (s) then $f_{0}^{\prime}$ becomes the Lie algebra automorphism induced from $f_{0}$. From this data we will construct an Anosov diffeomorphism $f: G / \Gamma \rightarrow G / \Gamma$. At this writing, this is the most general known construction of an Anosov diffeomorphism.

Since the linear automorphism $f_{0}^{\prime}: B \rightarrow \rightarrow B$ is hyperbolic, we get the usual invariant splitting $(5)=\left(5^{s}+\mathbb{S}^{2}\right.$. Furthermore, (see [114]) there exists constants $c, c^{\prime}$ such that $0<c<1<c^{\prime}$ and an inner product on (5) so that

$$
\begin{array}{ll}
\left\|f_{0}^{\prime}(v)\right\|<c\|v\| & \text { all } v \in \mathcal{B S}^{s}, \\
\left\|f_{0}^{\prime}(u)\right\|>c^{\prime}\|u\| & \text { all } u \in \mathcal{S}^{\prime} \text {. }
\end{array}
$$

Next by right translations, identifying $\$ 5$ with $T_{e}(G)$, the splitting and inner product are imposed on the tangent space of every point of $G$. For this Riemannian metric on $G$, it is easily checked that $f_{0}: G \rightarrow G$ is given a hyperbolic structure or that $f_{0}: G \rightarrow G$ is an Anosov diffeomorphism.

Furthermore, this splitting of $T(G)$ and the Riemannian metric are both invariant under the action of $G$ on $G$ given by right translation. In particular they are right invariant under $\Gamma$ and so $f_{0}$ induces an Anosov diffeomorphism $f$ on the compact coset space $G / \Gamma$.

For the existence of the $f_{0}$ in the previous construction, the next proposition shows that $G$ must be nilpotent.

(3.6) Proposition. Suppose that $\phi$ : (5) $\rightarrow$ (5) is a Lie algebra automorphism which is hyperbolic as a linear map. Then (s) must be nilpotent.

For a proof, A. Borel has given me the following reference: let (5) be a finite dimensional Lie algebra over a field having an automorphism no eigenvalue of which is a root of unity; then if is nilpotent. Exercise in Bourbaki with hints: Algebras de Lie, Ex. 21b, p. 124.

Now that we know that this construction forces $G$ to be nilpotent, and that $\Gamma$ is a uniform discrete subgroup, the results of Malcev [61], summarized in [12a], become important.

(3.7) Theorem (Malcev). (a) A necessary and sufficient condition for a discrete group $\Gamma$ to occur as a uniform subgroup of a simply connected nilpotent Lie group is that $\Gamma$ be a finitely generated nilpotent group containing no elements of finite order.

(b) A necessary and sufficient condition on a nilpotent simply connected Lie group $G$ that there exist a uniform discrete subgroup $\Gamma$ is that 
the Lie algebra of $G$ has rational constants of structure in some basis.

(c) If $\Gamma_{i}$ is a uniform discrete subgroup of a simply connected nilpotent group $G_{i}, i=1,2$, then any isomorphism $\Gamma_{1} \rightarrow \Gamma_{2}$ can be uniquely extended to an isomorphism $G_{1} \rightarrow G_{2}$.

The coset space $G / \Gamma, G, \Gamma$ as above is called a nilmanifold.

While (3.6) and (3.7) give some general perspective on our class of homogeneous space Anosov diffeomorphisms, this situation cannot be said to be completely understood. There certainly do exist, however, many nontoral examples of Anosov diffeomorphisms on nilmanifolds as special cases of the above construction. We give two of them now with $\operatorname{dim} G=6$.

Let $G_{1}, G_{2}$ be copies of the three dimensional simply connected, nonabelian nilpotent Lie group. We take a basis $X_{i}, Y_{i}, Z_{i}$ of $\mathbb{S}_{i}$, $i=1,2$ with the bracket relations $\left[X_{i}, Y_{i}\right]=Z_{i}, i=1,2$ and all other brackets zero. The main group $G$ of our basic construction above will be $G_{1} \times G_{2}$. For each real number $\lambda>1$ we define a hyperbolic automorphism $f_{0}$ of $G$ by specifying $f_{0}^{\prime}\left(f_{0}^{\prime},(\$),(5 s)\right.$, etc. as in the above construction) on (S in terms of the basis as follows.

$\begin{array}{ll}\text { EXAmPLE } 1 & \text { EXAMPLE } 2 \\ X_{1} \rightarrow \lambda X_{1} & X_{1} \rightarrow \lambda X_{1} \\ Y_{1} \rightarrow \lambda^{2} Y_{1} & Y_{1} \rightarrow \lambda^{-3} Y_{1} \\ Z_{1} \rightarrow \lambda^{3} Z_{1} & Z_{1} \rightarrow \lambda^{-2} Z_{1} \\ X_{2} \rightarrow \lambda^{-1} X_{2} & X_{2} \rightarrow \lambda^{-1} X_{2} \\ Y_{2} \rightarrow \lambda^{-2} Y_{2} & Y_{2} \rightarrow \lambda^{3} Y_{2} \\ Z_{2} \rightarrow \lambda^{-3} Z_{2} & Z_{2} \rightarrow \lambda^{2} Z_{2}\end{array}$

Note that in both examples brackets are preserved. In Example 1, one sees that (\$s ${ }^{u},\left(S^{s}\right.$ are both ideals which coincide with nilsubalgebras $B_{1}$ and $B_{2}$ respectively. In this case $G$ is the product of the corresponding subgroups, $G=G^{u} \times G^{s}$.

In Example 2, both $\left(5^{u}\right.$ and $(5 s s$ are seen to be abelian, but they are not ideals and $G$ is not (in the group sense) a product of the corresponding subgroups $G^{u}$ and $G^{s}$.

The next step is to find a uniform discrete subgroup $\Gamma \subset G$ such that $f_{0}(\Gamma)=\Gamma$. For this we will use matrices with coefficients in an algebraic number field. Let $K=Q\left(3^{1 / 2}\right)$, the number field of $3^{1 / 2}$ adjoined to the rationals, and $\sigma: K \rightarrow K$ the nontrivial Galois automorphism (sending $3^{1 / 2}$ into $-\left(3^{1 / 2}\right)$ ).

We may suppose that $\mathscr{S}_{1}$ and $\mathscr{S}_{2}$ are each represented by matrices of the form, 


$$
\left(\begin{array}{lll}
0 & X & Z \\
0 & 0 & Y \\
0 & 0 & 0
\end{array}\right) \quad X, Y, Z \in R
$$

and $B=B_{1} \times S_{2}$ becomes the space of matrices

$$
\left(\begin{array}{ll}
A & 0 \\
0 & B
\end{array}\right)
$$

$A, B$ each of the form (3.8). We will take $\Gamma_{0}$ then to be the lattice of (5) of matrices of the form

$$
\left(\begin{array}{cc}
A & 0 \\
0 & A^{\sigma}
\end{array}\right)
$$

where $A$ is as in (3.8) but $X, Y, Z$ are restricted to be algebraic integers in $K$ and $A^{\sigma}$ is the image of $A$ under the map induced by $\sigma: K \rightarrow K$.

Then if $\lambda=2+3^{1 / 2}, \lambda \lambda^{\sigma}=1$ and $f_{0}^{\prime}$ preserves $\Gamma_{0}$. We take $\Gamma$ to be the image of $\Gamma_{0}$ under the exponential map $\$ \rightarrow G$. Then it can be proved that $\Gamma$ is a uniform discrete subgroup of $G$ with $f_{0}(\Gamma)=\Gamma$. This finishes the description.

One can generalize the previous construction by using a diagonal process defined by the Galois automorphisms of an algebraic number field. See Weil [125] for this type of argument.

It seems possible that if $f \in \operatorname{Diff}(M)$ is Anosov, where $M$ is compact, then $M$ is covered by Euclidean space $\tilde{M}$, and that even the induced Anosov diffeomorphism on $\tilde{M}$ is topologically conjugate to a linear hyperbolic map. However, we have an example of an Anosov diffeomorphism $f: V \rightarrow V$ where $V$ is a simply connected complete Riemannian manifold, noncompact and not Euclidean space. This example goes as follows.

The construction starts with $G$ as $\operatorname{SL}(2, C)$ and proceeds something like our earlier nilpotent examples (but no $\Gamma$ this time).

Let $\phi: G \rightarrow G$ be the inner automorphism obtained by conjugation with the matrix

$$
A=\left(\begin{array}{ll}
\alpha & 0 \\
0 & \alpha^{-1}
\end{array}\right)
$$

in $G$ where $\alpha>1$. Then if $e^{a d A}:$ (5) $\rightarrow$ is the Lie algebra automorphism, we have the invariant decomposition $(B)=B^{3}+B^{2}+h$ where $\left(s^{s}\right.$ is contracting under $e^{a d A}$, (\$) ${ }^{u}$ expanding and $h$ is invariant pointwise. In fact $h$ is the Lie algebra of the centralizer $H$ of $A$, of all diagonal matrices. Just as in the previous construction we put a metric on 
$B=T_{e}(A)$ which is right translated around, but contains a degenerate component corresponding to $h$.

On $G / H$, however, the degeneracy is divided out so that we have an induced Anosov diffeomorphism $\phi_{0}: G / H \rightarrow G / H . G / H$ is a $4-$ dimensional manifold which is not contractible, but clearly simply connected.

Novikov informed me that he could prove that if $f \in \operatorname{Diff}(M)$ is Anosov, $M$ compact, where the dimension of $W^{u}(x)$ is one less than the dimension of $M$. then $\pi_{1}(M)$ is abelian and $M$ is covered by Euclidean space.

The two dimensional toral example was first communicated to me by Thom to show that there was an open set in $\operatorname{Diff}\left(T^{2}\right)$ of diffeomorphisms with no contracting periodic points, therefore implying that diffeomorphisms satisfying (2.2) were not dense. After adding some geometry to the example, I showed it to Anosov when I spoke on the examples of $\S I .5$ in the Soviet Union in 1961. By 1962 Anosov announced his theorem on structural stability in the context of what is called here Anosov diffeomorphisms. Proofs have now appeared [9].

The problem of the existence of (compact) nontoral diffeomorphisms was posed by Anosov in his Congress talk, Moscow 1966. Previously, after putting this problem into Lie group perspective, I had consulted many Lie group experts to arrive finally at what is here. In particular, conversations with Boothby, Borel, Hochschild, and Langlands were very helpful. The 6-dimensional Example 2 as well as the explicit algebraic number theory approach were given to me by Borel.

I.4. The zeta function of a diffeomorphism. Suppose $f: M \rightarrow M$ is a diffeomorphism with the property that $N_{m}<\infty, m=1,2, \ldots$ where $N_{m}=N_{m}(f)$ is the number of fixed points of $f^{m}$. This is a generic property (see §I.6). Then following Artin-Mazur [12], one defines the zeta function of $f$ as the formal power series $\zeta(t)=\exp \sum_{m=1}^{\infty}(1 / m) N_{n} t^{m}$. This turns out to be an interesting invariant of $f$. Of course $\zeta_{f}(t)=\zeta(t)$ is an invariant of the topological conjugacy class of $f$ and even of the conjugacy class "on $\Omega$ " of $f$.

The zeta function thus contains all the information about the numbers $N_{m}=N_{m}(f)$ where $N_{m}$ counts all the periodic points of period $m$. But this is different from $K_{m}=K_{m}(f)$ which denotes the number of periodic points of least period $m$. The number $K_{m}$ is more directly interesting in many respects and it is natural to ask for the relation between $N_{m}$ and $K_{m}$. From the definition it follows directly that 
(4.1) Proposition.

$$
\sum_{l \text { divides } m} K_{l}=N_{m}
$$

Narasimhan pointed out to me that one solves (4.1) for the $K_{l}$ by the Mobius inversion theorem (see [36]). This gives

(4.2) Proposition.

$$
K_{m}=\sum_{l \text { divides } m} \mu(l) N_{m / l}
$$

Here if $l=p_{1} \cdots p_{r}$ where the $p_{i}$ are distinct primes, then $\mu(l)$ $=(-1)^{r}, \mu(1)=1$, and if $l$ contains a power of a prime, $\mu(l)=0$. The function $\mu(l)$ is called the Möbius function.

Observe that $m$ always divides $K_{m}$ (i.e., $K_{m} / m \in Z^{+}$).

The inspiration for the above zeta function is the Weil zeta function of an algebraic variety over a finite field. Dwork recently proved the rationality of this zeta function, see [101] for a general reference.

For the differentiable version, there is the following theorem.

(4.3) Theorem (Artin-Mazur [12]). For any compact manifold, there is a dense set of $\operatorname{Diff}(M)$ for which the following etsimate holds:

$$
N_{m} \leqq C k^{m} \text {. }
$$

Here $C, k$ are positive constants which depend only on the diffeomorphism $f$ and $N_{m}=N_{m}(f)$.

(4.4) CoRollary. For a dense set of Diff $(M)$, the zeta function has a positive radius of convergence, so it can really be considered a function.

Actually Artin and Mazur define $N_{m}$ to be the number of isolated fixed points of $f^{m}$, while permitting $f^{m}$ to have an infinite number of fixed points. Thus, for example, they do not know whether the fixed point set is finite for the maps in the dense set they obtain.

The proof of (4.3) uses algebraic approximation techniques which go back to John Nash [73]. Actually Artin and Mazur define $\zeta(t)$ for differentiable maps for which $N_{m}<\infty$ and prove their theorem in the more general context of differentiable maps. The following problem then becomes important.

(4.5) Problem. Is $\zeta(t)$ generically rational (i.e., is $\zeta_{f}$ rational for a Baire set of $f$ )?

This goes beyond their theorem in two ways. First, generically true means true for a Baire set which, of course, is much bigger than sim- 
ply a dense set. Secondly, rationality is stronger than possessing a positive radius of convergence. Rationality is especially important because this means for the diffeomorphism that the poles and zeros of the zeta function, a finite number of invariants, determine the infinite set of $N_{m}$. The $N_{m}$ are of course very important objects to get ones hands on.

In the direction of (4.5), Artin and Mazur [12] asked if diffeomorphisms in their dense set have a zeta function which is algebraic.

More recently, there has been proved the following

(4.6) Theorem (K. Meyer). If $f \in \operatorname{Diff}(M), M$ compact, satisfies Axiom A (see §I.6), then the estimate of (4.3) is valid.

$\mathrm{K}$. Meyer's proof of this is very simple and if one had the density (see §I.6) for Axioms A and B, this would of course supersede (4.3).

We will now examine the zeta function for our examples.

If $f: M \rightarrow M$ is a diffeomorphism such that $N_{m}<\infty$ for all $m \in Z$ and $\Lambda$ is a closed invariant subset of $M$, then by definition $\zeta_{\Lambda}(f)$ $=\exp \sum_{m=1}^{\infty}(1 / m) N_{m}^{\prime} t^{m}$ where $N_{m}^{\prime}$ is the number of $x \in \Lambda$ such that $f^{m}(x)=x$.

(4.7) Proposition. Suppose for $f \in \operatorname{Diff}(M)$, the periodic points are all contained in the union of two disjoint closed invariant subsets $\Lambda_{1}, \Lambda_{2}$ of $M$. Suppose also that $\zeta_{\Lambda_{1}}$ and $\zeta_{\Lambda_{2}}$ are rational (or convergent). Then $\zeta_{f}=\zeta$ is rational (or convergent) and in fact $\zeta(t)=\zeta_{\Lambda_{1}}(t) \cdot \zeta_{\Lambda_{2}}(t)$.

PRoOF.

$\zeta(t)=\exp \sum \frac{N_{m}^{\prime}+N_{m}^{\prime \prime}}{m} t^{m}=\exp \sum \frac{N_{m}^{\prime}}{m} t^{m} \exp \sum \frac{N_{m}^{\prime \prime}}{m} t^{m}=\zeta_{\Lambda_{1}}(t) \cdot \zeta_{\Lambda_{2}}(t)$.

(4.8) Lemma from Calculus. $\log (1 /(1-y))=\sum_{k=1}^{\infty}(1 / k) y^{k}$.

For the diffeomorphisms of (2.2) the following theorem gives the zeta function.

(4.9) Theorem. Suppose for $f \in \operatorname{Diff}(M), \Omega$ is finite. Then clearly $\Omega=U_{\gamma \in P} \Omega_{\gamma}$ where $P$ is the set of periodic orbits of $f$, and $\Omega_{\gamma}$ is the set of points of $\Omega$ in $\gamma$. The zeta function of $f$ is the following, where $m(\gamma)$ $=$ period of $\gamma$,

$$
\zeta(t)=\prod_{\gamma \in P} \frac{1}{1-t^{m(\gamma)}} .
$$

Proof. By (4.8) $1 /\left(1-t^{m}(\gamma)\right)=\exp \sum_{k=1}^{\infty}(1 / k) t^{m(\gamma) k}$. Apply $(4.7)$. The Lefschetz number $L(p)=L(p, f)$ of a hyperbolic fixed point $p$ 
of $f \in \operatorname{Diff}(M)$ may be defined most simply, perhaps, as \pm 1 where the sign is the sign of $\operatorname{det}(I-D f(p)), I: T_{p}(M) \rightarrow T_{p}(M)$ being the identity.

A modern proof of a more general version of the following theorem of Lefschetz may be found in Dold [26].

(4.10) Lefschetz Trace Formula. Suppose $f \in \operatorname{Diff}(M)$ has only hyperbolic fixed points and Fix $(f)$ denotes the set of fixed points of $f$. Then

$$
\begin{gathered}
\sum_{p \in \text { Fix }(f)} L(p)=\Lambda(f) \text { where } \\
\Lambda(f)=\sum_{i=0}^{\operatorname{dim} M}(-1)^{i} \operatorname{Trace}\left(f_{*_{i}}: H_{i}(M, R) \rightarrow H_{i}(M, R)\right) .
\end{gathered}
$$

Here $f_{*_{i}}$ is the induced automorphism of the $i$ th homology group of $M$ with real coefficients.

The following proposition follows from the definition of $L(p)$ and the eigenspace decomposition of $D f(p)$. (One may assume $D f(p)$ to be semisimple in the proof.)

(4.11) Proposition. For $p \in \operatorname{Fix}(f), f \in \operatorname{Diff}(M), L(p)=(-1)^{u} \Delta$, where $u=\operatorname{dim} W^{u}(p)$ and $\Delta=+1$ if $f$ preserves orientation on $W^{u}(p)$ and $\Delta=-1$ if $f$ reverses it.

The following is well known and will be useful in computing the zeta function for some of the Anosov diffeomorphisms.

(4.12) Proposition. Suppose $f \in \operatorname{Diff}(M)$ is such that for every $m \in Z^{+}$ and every $x \in \mathrm{Fix}\left(f^{m}\right), L\left(x, f^{m}\right)=+1$. Then $\zeta(t)=\prod_{i=1}^{\operatorname{dim} M} \zeta_{i}(t)^{(-1)^{i}}$ where

$$
\zeta_{i}(t)=\prod_{j}\left(1-\lambda_{i j} t\right)^{-1} \quad \text { and } \quad \lambda_{i j}, j=1, \cdots, \operatorname{dim} H_{i}(M, R),
$$

are the eigenvalues (generalized and counted with multiplicity) of $f_{*_{i}}: H_{i}(M, R) \rightarrow H_{i}(M, R)$.

Proof. By (4.10)

$$
\begin{aligned}
N_{m}(f) & =\sum_{i=0}^{\operatorname{dim} M}(-1)^{i} \operatorname{Trace}\left(f^{m}\right)_{*_{i}}, \quad m=1,2,3, \cdots \\
& =\sum_{i=0}^{\operatorname{dim} M}(-1)^{i} \sum_{r=1}^{\operatorname{dim} H_{i}} \lambda_{r i}^{m} .
\end{aligned}
$$

So we obtain 


$$
\log \zeta(t)=\sum_{m=1}^{\infty} \frac{1}{m} \sum_{i=0}^{\operatorname{dim} M}(-1) \sum_{r=1}^{i \operatorname{dim} H_{i}} \lambda_{r i}^{m} m
$$

or

$$
\zeta(t)=\prod_{i=0}^{\operatorname{dim} M} \zeta_{i}(t)^{(-1)^{i}}
$$

where

$$
\begin{aligned}
\log \zeta_{i}(t) & =\sum_{m=1}^{\infty} \frac{1}{m} \sum_{r=1}^{\operatorname{dim} H_{i}}\left(\lambda_{i r} t\right)^{m} \\
& =\sum_{r=1}^{\operatorname{dim} H_{i}} \sum_{m=1}^{\infty} \frac{1}{m}\left(\lambda_{i r} t\right)^{m} \\
\log \zeta_{i}(t) & =\sum_{r=1}^{\mathrm{dim} H_{i}} \log \left(1-t \lambda_{i r}\right)^{-1} \text { by }
\end{aligned}
$$

and

$$
\zeta_{i}(t)=\prod_{r=1}^{\mathrm{dim} H_{i}}\left(1-t \lambda_{i r}\right)^{-1}
$$

For any $f \in \operatorname{Diff}(M)$, the function $\zeta(t)$ defined in (4.12) is well defined even though $L\left(x, f^{m}\right)$ is not always 1 . It will be called the false zeta function of $f$ and denoted by $\tilde{\zeta}(t)$ or $\zeta_{f}(t)$. It is rational and its expansion counts the periodic points algebraically. In fact, the whole difficulty of the problem of the rationality of the (honest) zeta function is that it counts the periodic geometrically, not algebraically. Proposition (4.12) shows that under the condition $L\left(x, f^{m}\right)=1$ for all $x \in \operatorname{Fix}\left(f^{m}\right)$, and $m \in Z^{+}$, the false and honest zeta functions coincide.

Note that if $\tilde{N}_{m}$ is the number of points of $f$ of period $m$ counted algebraically, i.e., $\tilde{N}_{m}=\sum_{x \in \operatorname{Fix}\left(f^{m}\right)} L\left(p, f^{m}\right)$, then (4.12) shows that $\tilde{\zeta}(t)=\exp \sum_{m=1}(1 / m) \tilde{N}_{m} t^{m}$ and one can see how the following theorem of Fuller [31] fits into this context (see also [38]).

(4.13) Theorem. Suppose $h: L \rightarrow L$ is a homeomorphism of a polyhedron of nonzero Euler characteristic. Then $h$ has a periodic point.

Otherwise all the $\tilde{N}_{m}$ would be zero and $\tilde{\zeta}$ would be one. But the degree of $\tilde{\zeta}$ is minus the Euler characteristic (from (4.12)).

(4.14) Proposition. Suppose $f: M \rightarrow M$ is an Anosov diffeomorphism such that the corresponding expanding bundle $E^{u}$ is orientable. Then $\zeta_{f}$ is rational and 
(a) if $D f: E^{u} \rightarrow E^{u}$ is orientation preserving then

$$
\begin{array}{ll}
\zeta_{f}=\tilde{\zeta}_{f} \quad \text { if } \operatorname{dim} \text { fiber } E^{u} \text { is even, } \\
\zeta_{f}=1 / \tilde{\zeta}_{f} & \text { if } \operatorname{dim} \text { fiber } E^{u} \text { is odd, }
\end{array}
$$

(b) if $D f: E^{u} \rightarrow E^{u}$ is orientation preserving then

$$
\begin{array}{ll}
\zeta_{f}(t)=\tilde{\zeta}(-t) & \text { if } \operatorname{dim} \text { fiber } E^{u} \text { is even, } \\
\zeta_{f}(t)=1 / \tilde{\zeta}(-t) & \text { if } \operatorname{dim} \text { fiber } E^{u} \text { is odd. }
\end{array}
$$

This follows directly from (4.11) and (4.12).

It seems likely that looking at a double covering of $M$, one could furthermore prove that the zeta function of every Anosov diffeomorphism was rational.

For the toral case of $\S I .3$ defined by hyperbolic $f_{0} \in \operatorname{GL}(n, Z)$, one finds the zeta function defined explicitly in terms of the eigenvalues of $f_{0}$. In this case $f_{0}$ coincides with the automorphism of $H^{1}\left(T^{n}, Z\right)$ induced by $f: T^{n} \rightarrow T^{n}$. By the Kunneth formula the whole cohomology ring of $T^{n}$ is given as a tensor product of $H^{*}\left(T^{1}\right)$ and so one obtains all of the eigenvalues of $f^{*}: H^{*}\left(T^{n}\right) \rightarrow H^{*}\left(T^{n}\right)$ as products of the eigenvalues of $f_{0}$. One thus obtains easily via (4.14)

(4.15) Proposition. For the toral diffeomorphism $f: T^{n} \rightarrow T^{n}$ defined by hyperbolic $f_{0} \in \mathrm{GL}(n, Z)$ with the eigenvalues $\lambda_{1} \cdots \lambda_{n}$ of $f_{0}$, we have:

(a) $\Lambda\left(f^{m}\right)=\prod_{i}\left(1-\lambda_{i}^{m}\right)$,

(b) $\tilde{\zeta}(t)=\prod\left(1-\lambda_{i_{1}} \lambda_{i_{2}} \cdots \lambda_{i_{k}} t\right)^{(-1)^{k+1}}$,

$$
\text { all }\left(i_{1}, \cdots, i_{k}\right) \ni 1 \leqq i_{1}<i_{2}<\cdots<i_{k} \leqq n,
$$

(c) $\zeta(t)$ is defined from $\tilde{\zeta}(t)$ according to (4.14) where one checks the appropriate case from the $\lambda_{i}$ with $\left|\lambda_{i}\right|>1$.

Finally, we remark that through the work of Matsushima [63], Mal'cev [61], Nomizu [75], and Kostant [54], one can compute the zeta functions for the nilpotent examples of $\$ I .3$ quite explicitly.

I.5. Shift automorphisms and homoclinic points. From the preceding sections, one might ask whether the set $\Omega$ of nonwandering points must be a manifold generically (allowing certainly for components to have varying dimensions). The examples of $\$ \S I .2$ and 3 have this property. Here we will see that the answer is no, and in fact give an example of a diffeomorphism of $S^{2}, \Omega$-stable, such that $\Omega$ is the union of a Cantor set and two isolated points.

First a description of the shift automorphism of symbolic dynamics will be given (see [14] or [35a] for more details). Let $S$ be a finite set, 
discrete topology, consisting of $N$ elements and define $X_{S}$ to be the set of functions from $Z$ to $S$ provided with the compact open topology ( $Z$ has the discrete topology). If $a \in X_{S}$, the value of $a$ at $m \in Z$ will be denoted by $a_{m}$ and we write $a=\prod a_{m}$. Then $a$ may be thought of as a doubly infinite sequence of elements of $S$ with a decimal point between $a_{0}$ and $a_{1}$, thus a $a=\left(\cdots a_{-1} a_{0} \cdot a_{1} a_{2} \cdots\right)$. An important special case is where $S$ has two elements and here we could assume each $a_{i}$ is either 0 or 1 . For general $S, X_{S}$ is homeomorphic to a Cantor set.

Define a map $\alpha: X_{S} \rightarrow X_{S}$ by $(\alpha(a))_{m}=a_{m+1}$. In terms of the doubly infinite sequences, $\alpha$ shifts the decimal point one place to the right. It is easily seen that $\alpha$ is a homeomorphism, called the shift automorphism of $X_{S}$. It has been widely studied in ergodic theory and probability [14] as well as topological dynamics [35a].

(5.1) Propositron. The periodic points of $\alpha$ are dense in $X_{S}$ and if $C_{k}$ is the number of periodic points of period $k$ (i.e. fixed points of $\alpha^{k}$ ) $k>0$ then $C_{k}=N^{k}$ where $N$ is the cardinality of $S$.

Proof. The element $a=\prod a_{m} \in X_{S}$ will be periodic of period $k$ precisely when $a_{m}=a_{m+k}$ for all $m \in Z$. Thus it is determined by $a_{1}, \ldots, a_{k}$ with $a_{1}, \cdots, a_{k}$ arbitrary elements of $S$. Given any $b=\prod b_{m} \in X_{S}$ and $K$ large, one can choose a periodic approximation $a=\prod a_{m}$ of $b$ with $a_{i}=b_{i}$ for $|i|<K$. The proposition follows.

(5.2) Corollary. The zeta function for $\alpha: X_{S} \rightarrow X_{S}$ can be defined as in $\S 1.4$ and in fact $\zeta(t)=1 /(1-N t)$ where $N=$ cardinality of $S$.

This follows from (5.1) with the aid of (4.8).

M. Morse has proved (see [35a]) that there is a subset of $X_{S}$, homeomorphic to a Cantor set, which is a minimal set for $\alpha$.

To see how symbolic dynamics enters into our diffeomorphism problem, we will first describe an example of a diffeomorphism $g$ mapping a subset $Q$ of the plane in to the plane. Here $g(Q)$ is not a subset of $Q$, but eventually we will use $g$ to define a global diffeomorphism $f$ of $S^{2}$ onto itself. One might think of $Q$ as a neighborhood (not invariant) of an indecomposable piece of the nonwandering points of this $f: S^{2} \rightarrow S^{2}$.

Take then $Q$ to be a square in the plane $R^{2}$, for example, $Q=\left\{(x, y) \in R^{2}|| x|\leqq 1| y \mid, \leqq 1\right\}$. Then $g$ will map $Q$ into the region bounded by dotted lines with $g(A)=A^{\prime}$ etc. in Figure 1 . 


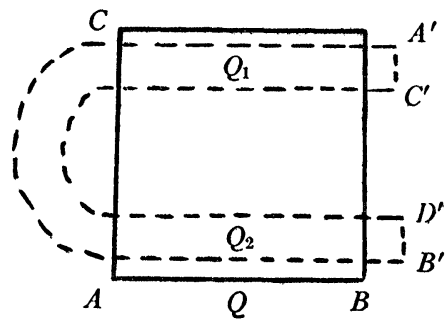

FIGURE 1

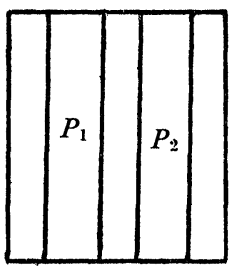

FIGURE 2

We will take any such $g$ which has the following properties.

(a) $g$ is a diffeomorphism of $Q$ on to the region in Figure 1 bounded by dotted lines sending $A \rightarrow A^{\prime}, B \rightarrow B^{\prime}$ etc.

(b) on each component $P_{1}, P_{2}$ of $g^{-1}(g(Q) \cap Q), g$ will be a linear map (up to a translation).

To understand (b) note that as a consequence of it, $P_{1}, P_{2}$ will be as in Figure 2 and $g\left(P_{i}\right)=Q_{i}, i=1,2$.

The reader will be able to verify that the intersections of all the images $g^{m}(Q), m=1,2, \cdots$ or, more accurately, $\bigcap_{m=1}^{\infty} g^{m}\left(Q^{(m)}\right)$ where $Q^{(m)}=Q \cap$ image $g^{m-1}$, is a product of a Cantor set and the interval $|x| \leqq 1$.

Define $\Lambda$ to be the intersection, $\bigcap_{m \in Z} g^{m}\left(Q^{(m)}\right), Q_{0}=Q, Q^{(m)}$ as above for $m>0$ and for $m<0, Q^{(m)}=g^{m}\left(Q^{(m+1)}\right)$. Thus $\Lambda$ may be thought of as the set of nonwandering points of $g: Q \rightarrow R^{2}$.

The careful reader will be able to check for himself the next proposition (which is in [115]).

(5.3) Proposition. The subset $\Lambda$ of $Q$ is compact, invariant under $g$, indecomposable and on $\Omega, g$ is topologically conjugate to the shift automorphism $\alpha: X_{S} \rightarrow X_{S}$, with the cardinality of $S=2$.

Furthermore one can prove stability with the less obvious proposition [115].

(5.4) Proposition. For a perturbation $g^{\prime}$ of $g, \Lambda^{\prime}$ defined similarly is also compact and invariant under $g^{\prime}$. Then $g^{\prime}: \Lambda^{\prime} \rightarrow \Lambda^{\prime}$ is also topologically conjugate to the shift $\alpha: X_{S} \rightarrow X_{S}$.

Thus (at least after we globally extend $g$ ) we have another example of a stable indecomposable piece of nonwandering points.

One may modify the above example in the following way. The image $g(Q)$ may wind half-way around $Q$ before passing through $Q$ the second time, or even wind around $Q$ several times for that matter (Figures 3 and 4). This won't change $g: \Lambda \rightarrow \Lambda$, but $g$ will be different 
on $U(\Lambda)$ where $U$ is any neighborhood of $\Lambda$. The intrinsic picture (with respect to $\Omega$ ) is the same for Figures $1,3,4$ but they differ extrinsically (in any neighborhood of $\Omega$ ).

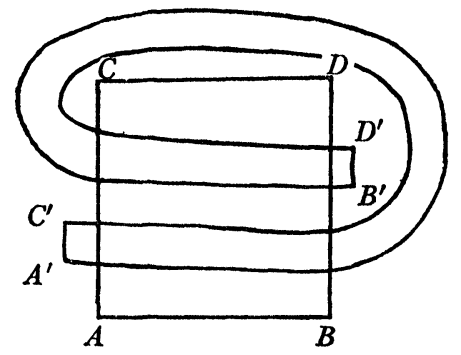

FIGURE 3

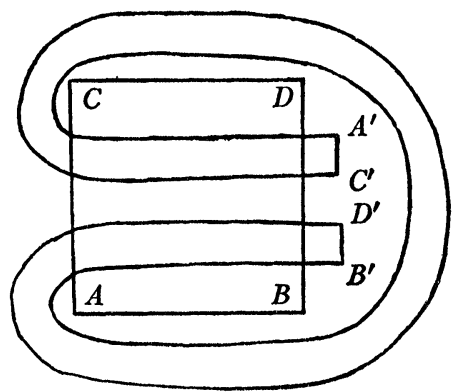

FIGURE 4

One may further modify the above examples by having $g(Q)$ passing through $Q$ several times (see Figures 5 and 6).

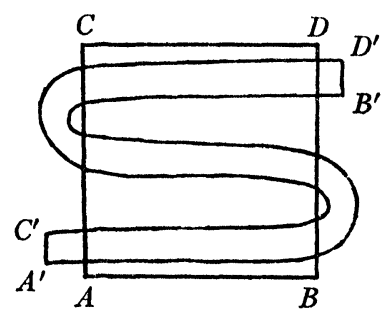

Figure 5

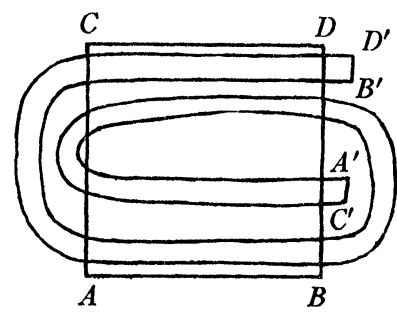

FIGURE 6

In all of these examples it is important to keep the linearity condition (b) above. Then one may define and analyze $\Lambda$ as in the first case. Always $g: \Lambda \rightarrow \Lambda$ will be topologically conjugate to a shift automorphism $\alpha: X_{S} \rightarrow X_{S}$ and stably so. The cardinality of $S$ will equal the number of components in $Q \cap g(Q)$, e.g., three for Figure 5 and four for Figure 6. Thus all of the shift automorphisms occur in the above framework.

To really complete this picture, $\Lambda$ above must appear as an indecomposable piece of the nonwandering points of a global diffeomorphism. We construct such an $f: S^{2} \rightarrow S^{2}$ now which extends the map $g: Q \rightarrow R^{2}$ of Figure 1. Consider Figure 7 .

We have put the square $Q$ into a disk $D^{2} \subset R^{2}$ and we extend $g$ to $g_{0}: D^{2} \rightarrow D^{2}$ by mapping $G$ diffeomorphically on to $G^{\prime}$ and $F$ onto $F^{\prime}$. The map $g_{0}: F \rightarrow F^{\prime}$ is defined so that it is a contraction about some fixed point $p_{0}$ in $F^{\prime}$. This $g_{0}: D^{2} \rightarrow D^{2}$ will be a diffeomorphism of $D^{2}$ onto a subset of $D^{2}$ so that the nonwandering set is the disjoint union of $\Lambda$ and $p_{0}$. Finally one easily extends $g_{0}$ to $f: S^{2} \rightarrow S^{2}$ so that the non- 


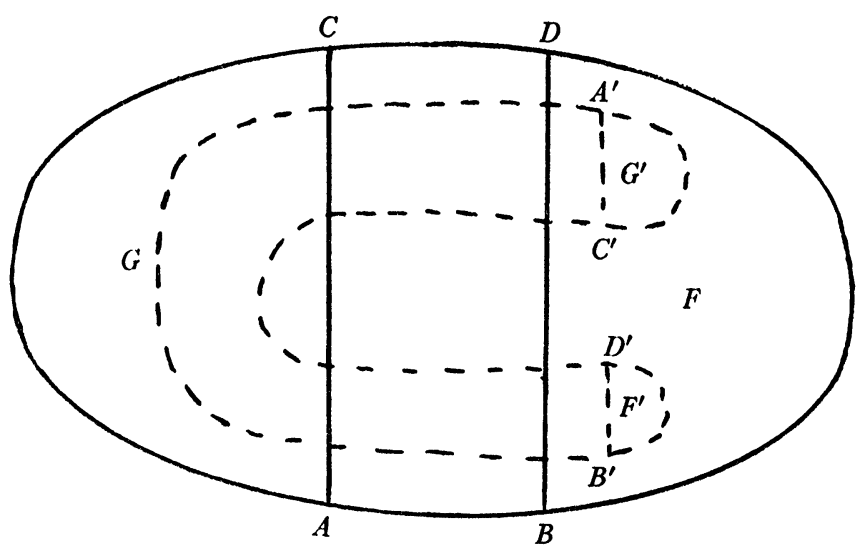

Figure 7

wandering points $\Omega=\Omega(f)=\Lambda \cup_{p_{0}} \cup q_{0}$ where $q_{0}$ is an expanding fixed point of $f$ outside of $D^{2}$. This $f$ is our desired global diffeomorphism.

At this point it seems appropriate to give a general way of constructing $\Omega$-stable diffeomorphisms of $S^{2}$.

Take any diffeomorphism $f: S^{2} \rightarrow S^{2}$ satisfying (2.2) with a contracting fixed point $p$. Let $V$ be a contracting disk neighborhood of $p$ and redefine $f$ on $V$ to be $g_{0}$ as described above (the "surgery" of $\$ I .10$ ). More generally let $f: S^{2} \rightarrow S^{2}$ satisfy (2.2) with a contracting periodic orbit $p_{1}, \cdots, p_{k}$ and let $V$ be a disk neighborhood of $p_{1}$ such that $f^{k}$ contracts $V$ into its interior. Then one modifies $f$ (via surgery again) on $\bigcup_{i=0}^{k-1} f^{i}(V)$ to obtain $f^{\prime}: S^{2} \rightarrow S^{2}$ so that on $V, f^{\prime k}$ is conjugate to $g_{0}$ above.

Finally a straightforward modification of the previous construction allows one to introduce into any diffeomorphism $f: S^{2} \rightarrow S^{2}$ satisfying (2.2), indecomposable pieces $\Lambda$ topologically conjugate to shift automorphisms on $N$ symbols (cardinality $S=N$ ) where $N$ can be anything we like.

In all of these examples the indecomposable pieces of $\Omega$ are shift automorphisms, finite periodic orbits or products of the two. We see easily from previous remarks that the zeta functions of these $f: S^{2} \rightarrow S^{2}$ are finite products of factors of the form $1 /\left(1-N t^{q}\right)$ where $N$ and $q$ are positive in tegers.

It should be noted that the Lefschetz Trace Formula imposes conditions on what products of the above form can occur in these zeta functions. It restricts the $N_{i}, p_{i}$ that can occur in $\zeta(s)$ $=\prod_{i=1}^{k}\left(1-N_{i} t^{p_{i}}\right)^{-1}$.

One can see an analogy between the shift automorphism and the nilmanifold examples of $\S I .3$ by considering the shift automorphism in the following light. 
Let $Z_{n}$ be the cyclic group with $n$ elements and for each $m \in Z$ let $G_{m}$ be the abelian group of formal power series (starting at $m$ ), $f(x)=\sum_{i=m}^{\infty} a_{i} x^{i}$ with $a_{i} \in Z_{n}$. Put the structure of a compact group on $G_{m}$ with the product topology. Define the locally compact group of all power series by $G=\bigcup_{m \in Z} G_{m}$. The map $\phi^{s}: G \rightarrow G$ defined by $f \rightarrow x f$ is a contraction while $\phi^{u}: G \rightarrow G$ defined by $f \rightarrow x^{-1} f$ is an expansion.

It is easily checked that the subgroup $\Gamma$ of $G \times G$ defined by $\Gamma=\left\{(f, \tilde{f}) \mid f(x)\right.$ a polynomial in $\left.G, f(x)=\sum_{i=m}^{k} a_{i} x^{i}, \tilde{f}(x)=\sum a_{-i} x^{i}\right\}$ is uniform (compact quotient) and discrete. The "hyperbolic" automorphism $\phi^{s} \times \phi^{u}: G \times G \rightarrow G \times G$ preserves $\Gamma$ and the induced homeomorphism $\phi: G / \Gamma \rightarrow G / \Gamma$ is precisely the shift automorphism on $n$ symbols.

One may identity $\phi^{s}: G \rightarrow G$ above with $g: \breve{W}^{s}(p) \rightarrow \breve{W}^{s}(p)$ where $p \in \Lambda, \Lambda$ as in the example of Figure $1, n=2, \breve{W}^{s}(p)=W^{s}(p) \cap \Lambda$.

There is a very close relation between the shift automorphisms discussed above and what are called homoclinic points, first discovered (in the restricted 3-body problem) and named by Poincare [90].

A homoclinic point of $f \in \operatorname{Diff}(M)$ is a point of intersection $x \in W^{s}(p)$ $\cap W^{u}(q)$. If $W^{s}(p)$ and $W^{u}(q)$ are transversal at $x$, then $x$ will be called a transversal homoclinic point.

As realized by Poincaré [90], homoclinic points complicate the orbit structure of a diffeomorphism considerably. The orbit of a homoclinic point consists (clearly) of homoclinic points. Taking the case $p=q$, one sees that the existence of homoclinic $x$ forces $W^{u}(p)$ to double back on itself oscillating faster and faster as it does so. For example, for the plane, we will obtain behavior something like that described in Figure 8.

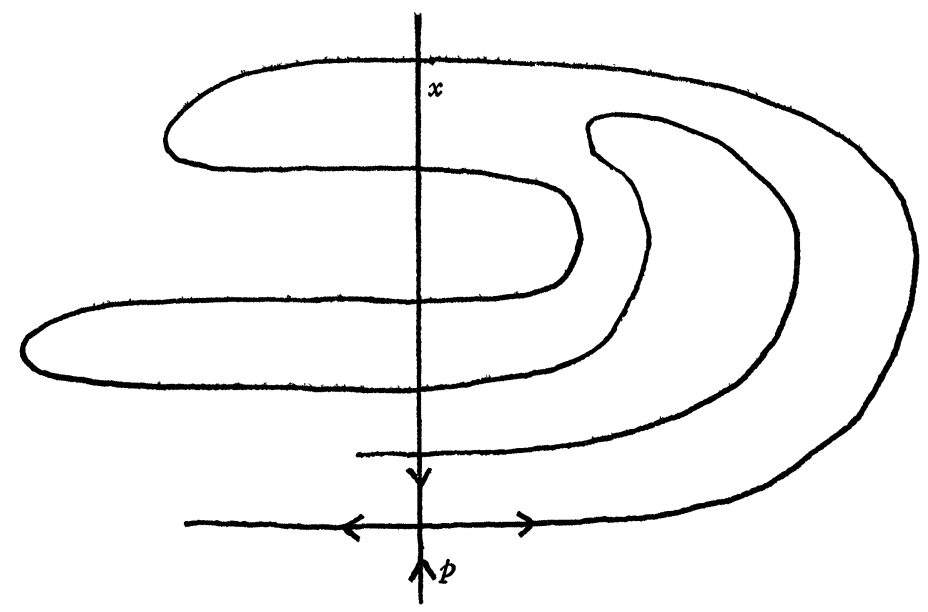

Figure 8 
This is the same phenomena that is occurring in Figure 1, but looked at in a different way. In fact, the best way to understand what is going on in Figure 8 is to imbed it (in some sense) in Figure 1. A great advantage of the horseshoe approach of Figure 1 is that one gets a satisfactory picture of the orbit structure and stability while a given homoclinic point at first glance seems to defy analysis. That is the idea behind the following theorem [115].

(5.5) THEOREM. Suppose $x$ is a transversal homoclinic point of $f \in \operatorname{Diff}(M)$. Then there is a Cantor set $\Lambda \subset M, x \in \Lambda$, and $m \in Z^{+}$such that $f^{m}(\Lambda)=\Lambda$ and $f^{m}$ restricted to $\Lambda$ is topologically a shift automorphism.

By (5.1) this implies:

(5.6) Corollary. In every neighborhood of a transversal homoclinic point of $f \in \operatorname{Diff}(M)$, there is a periodic point.

We interpolate a little curiosity. Note that for the shift on $\lambda$ symbols, $N_{m}=\lambda^{m}$ so that by (4.2) and the fact that $K_{m} / m \in Z^{+}, K_{m}$ $=\sum_{l / m} \mu(l) \lambda^{m / l} \equiv 0 \bmod m$ for every $\lambda, m \in Z^{+}$. This number theoretic identity for $m$ a prime becomes $\lambda^{p} \equiv \lambda \bmod p$, or Fermat's Theorem.

The material in this section is mainly taken from [115] with a number of examples and figures added. The shift automorphism goes back to Hadamard (but it is even sometimes called the Bernoulli automorphism!) who used it to study geodesic flows on 2 manifolds of constant negative curvature [40]. M. Morse [69] obtained further results in the same context.

G. D. Birkhoff [17], [18] in his works on surface diffeomorphisms, studied homoclinic points. In [17], Birkhoff proved (5.6) in dimension 2 , and in his $[18$, p. 184] he noted a resemblance between his homoclinic points and Hadamard's shift automorphism.

I came across the "horseshoe" of Figure 1 when I was trying to get a geometric picture of a variant of van der Pol's equation in N. Levinson's paper [58]. He had written me earlier that this equation had stably an infinite number of periodic solutions. The "horseshoe" was the first example of a structurally stable (or $\Omega$-stable) diffeomorphism with an infinite number of periodic points [113].

Putting the shift automorphism into the group theoretic framework was done with the aid of Cal Moore.

I.6. Unification. The work of the present section is motivated by the search for unity in the examples and phenomena of the preceding part of the paper. Anosov's work on hyperbolic structures on manifolds gives a clue on how to proceed. The Anosov diffeomorphisms, 
however, are rare among all diffeomorphisms; only certain manifolds even permit them. One is looking for a class of diffeomorphisms which include all of the previous examples in a transparent way and will at least have the possibility of including an open dense subset of Diff $(M)$ for each compact manifold $M$. This is provided by diffeomorphisms described now, i.e., those satisfying Axioms A and B below.

Suppose then $f: M \rightarrow M$ is a diffeomorphism of a Riemannian manifold and $\Lambda \subset M$ is a closed invariant subset. We will say that $\Lambda$ is hyperbolic (or has a hyperbolic structure) if the tangent bundle of $M$ restricted to $\Lambda, T_{\Lambda}(M)$ has an invariant (continuous) splitting under $D f: T_{\Lambda}(M) \rightarrow T_{\Lambda}(M), T_{\Lambda}(M)=E^{u}+E^{s}$ such that $D f: E^{s} \rightarrow E^{8}$ is contracting and $D f: E^{u} \rightarrow E^{u}$ expanding (see $\S I .3$ for these definitions). The dimension of the fiber of $E^{s}$ need not be constant but only locally so. Since $\Lambda$ is invariant, $D f$ is an automorphism of the bundle $T_{\Delta}(M)$ and this with the Riemannian metric gives sense to the above definition. Note that if $\Lambda$ (or $M$ ) is compact, one may dispense with the Riemannian metric by (3.1).

The simplest examples of hyperbolic sets for diffeomorphisms are first of all the hyperbolic fixed points ( $\$ 1.2)$ and the hyperbolic periodic points. The finite union of these cover the case of $\Lambda$ finite. Next, of course, the Anosov diffeomorphisms of $\S 1.3$ are examples where the whole compact manifold is hyperbolic. Also for the examples of $\S I .5$, the $\Lambda$ homeomorphic to a Cantor set is easily checked to have a hyperbolic structure. In all of the above examples the hyperbolic sets consist of nonwandering points and the periodic points are dense in each of them (up to the unsolved problem (3.4)). The following is an example to show that hyperbolic sets need not satisfy either of these properties.

Take a diffeomorphism satisfying (2.2) which has a heteroclinic point $x \in W^{s}(p) \cap W^{u}(q)$. The 2-dimensional example of Figure 5, $\S I .2$, will do. Thus $\Omega$ is hyperbolic and one may extend this hyperbolic structure to the orbit of $x$. In fact the tangent spaces of $W^{s}(p)$ and $W^{u}(q)$ at $x$ give the desired splitting at $x$ and similarly for each point in the orbit of $x$. The orbit of $x$ together with $\Omega$ is a closed invariant set and this gives the example. The closure of the orbit of $x$ is an indecomposable hyperbolic set.

Recall that a homeomorphism $h: X \rightarrow X$ is said to be topologically transitive if there is a dense orbit. Then the dense orbits form a Baire set of $X$ (assuming that $X$ is a compact metric space).

We will now consider a diffeomorphism $f: M \rightarrow M$ of a compact manifold which satisfies the following two properties [116]. 
(6.1) Aхіом A: (a) the nonwandering set $\Omega$ is hyperbolic. (b) the periodic points of $f$ are dense in $\Omega$.

Of these two properties (a) is the most important in what follows. In fact (b) may even be a consequence of (a). The above example however shows that a proof of $(a) \Rightarrow$ (b) must use the fact that $\Omega$ consists of nonwandering points.

(6.2) Theorem (Spectral Decomposition of Diffeomorphisms) [117]. Suppose $f: M \rightarrow M$ satisfies (6.1). Then there is a unique way of writing $\Omega$ as the finite union of disjoint, closed, invariant indecomposable subsets (or "pieces") on each of which $f$ is topologically transitive:

$$
\Omega=\Omega_{1} \cup \ldots \cup \Omega_{k} \text {. }
$$

(6.3) Corollary. If $f: M \rightarrow M$ is as above one can write $M$ canonically as a finite disjoint union of invariant subsets $M=\bigcup_{i=1}^{k} W^{s}\left(\Omega_{i}\right)$ where $W^{s}\left(\Omega_{i}\right)=\left\{x \in M \mid f^{m}(x) \rightarrow \Omega_{i}, m \rightarrow \infty\right\}$.

As the remarks at the beginning of this section indicate, the examples of $\S \S I .2,3$ and 5 satisfy (6.1).

The spectral decomposition theorem gives a little perspective on the question of rationality of the zeta function. The zeta function of such an $f$ will be a product of zeta functions, one for each $\Omega_{i}$. It seems plausible to me that each of these zeta functions is rational. The results of $\S \S I .4$ and I.5 are consistent with this.

We explain why we use the words "Spectral Decomposition for Diffeomorphisms" in (6.2). The decomposition of the manifold into invariant sets of the diffeomorphism is quite analogous to the decomposition of a finite dimensional vector space in to eigenspaces of a linear map. In one case we are considering automorphisms in the category of differential topology, in the other, finite dimensional vector spaces.

As Jacques Tits pointed out, one may make this more precise by actually putting a linear transformation (generically) into the framework of (6.2). Suppose then $u: V \rightarrow V$ is a linear transformation of a complex $n$-dimensional vector space. By multiplying by a constant, we may suppose $u$ has determinant 1 , i.e., $u \in \operatorname{SL}(n, C)$. We will furthermore suppose that the eigenvalues $\lambda_{1}, \cdots, \lambda_{n}$ of $u$ have distinct absolute values which are not one. Consider the induced diffeomorphism of projective space $u_{0}: P^{n-1}(C) \rightarrow P^{n-1}(C)$ defined on coordinates by $Z_{i} \rightarrow \lambda_{i} Z_{i}$. Then $u_{0}$ will satisfy (2.2) with $\Omega$ consisting of $n$ fixed points $(0 \cdots 010 \cdots 0)$. The two ways of looking at the spectral decomposition coincide. 
Now we state the second of our two main axioms (introduced in [117]).

(6.4) Axıom B. Suppose that $f \in \operatorname{Diff}(M)$ satisfies Axiom A and that $\Omega_{i}, W^{s}\left(\Omega_{i}\right)$, etc. are as in (6.1), (6.2), (6.3). Then if $W^{s}\left(\Omega_{i}\right)$ $\cap W^{u}\left(\Omega_{j}\right) \neq \varnothing$, there exist periodic points $p \in \Omega_{i}, q \in \Omega_{j}$ such that $W^{s}(p)$ and $W^{u}(q)$ have a point of transversal intersection.

The following generalizes the theorem of Palis, see [82] and §I.2.

(6.5) Theorem. The set of $f \in \operatorname{Diff}(M)$ which satisfy Axioms A and $\mathrm{B}$ are open and such $f$ are $\Omega$-stable.

Assuming $f, \Omega_{i}$, etc. as above, we say that $\Omega_{i} \leqq \Omega_{j}$ if $W^{s}\left(\Omega_{i}\right)$ $\cap \mathrm{W}^{u}\left(\Omega_{j}\right) \neq \varnothing$. Then we also have (generalizing theorems of $\S 2$ )

(6.6) Theorem. If $f \in \operatorname{Diff}(M)$ satisfies Axioms $\mathrm{A}$ and $\mathrm{B}$, then $\leqq$ is a partial ordering which is preserved under perturbation.

These theorems (6.2), (6.3), (6.5), (6.6) have no proofs in the literature, but we will try to give a good sketch of their proofs in $\$ \S I .7$ and $8, \S I .7$ for (6.2) and (6.3), §I.8 for (6.5) and (6.6).

We say that $\Omega_{i_{1}} \leqq \Omega_{i_{2}} \leqq \Omega_{i_{3}} \leqq \cdots \leqq \Omega_{i_{n}}$ is a maximal chain if the $\Omega_{i_{j}}$ are distinct, and $n$ is maximal.

For every $f \in \operatorname{Diff}(M)$ satisfying Axioms $\mathrm{A}$ and $\mathrm{B}$, we define the diagram $\Delta(f)$ as follows. $\Delta(f)$ is a linear graph whose vertices correspond to the $\Omega_{i}$, labeled by conjugacy class, and directed 1 -simplices join consecutive vertices of maximal chains. The diagram $\Delta(f)$ is invariant under perturbations of $f$. Generalizing problem (2.4) is

(6.6)a Problem. What diagrams can occur for diffeomorphisms satisfying Axioms $\mathrm{A}$ and $\mathrm{B}$ ? Given first the manifold $M$, what diagrams can occur for $f \in \operatorname{Diff}(M)$ satisfying Axioms $\mathrm{A}$ and B? Finally one can label the diagrams with conjugacy classes of germs of diffeomorphisms on neighborhoods of the $\Omega_{i}$ as in $\S I .2$ and ask the above two questions for these labeled diagrams.

One can see that a prototype of diffeomorphisms satisfying Axioms $\mathrm{A}$ and $\mathrm{B}$ are those of $\$ \mathrm{I} .2$ with Axiom 3 replaced by Axiom $3^{\prime}$ there. The above results may be construed as saying that we have succeeded in relaxing the hypothesis that $\Omega$ is finite. The diagram here gives sort of a very generalized gradient structure to these diffeomorphisms.

The main point of Axioms $\mathrm{A}$ and $\mathrm{B}$ and subsequent theorems is that the hypotheses include and unify all known $\Omega$-stable diffeomorphisms, while describing an open set of $\operatorname{Diff}(M)$ which is amenable to study. In fact the above theorems as well as those in the future sections give the beginnings of a structure theory for diffeomorphisms satisfying Axioms A and B. 
Thus the question, "are these diffeomorphisms dense in Diff $(M)$," becomes particularly sharp. This is not yet settled. In this direction, the first theorem is

(6.7) Theorem. For compact $M$, the following properties of $f \in \operatorname{Diff}(M)$ are generic:

(a) Every periodic point is hyperbolic.

(b) For each pair of periodic points $p, q \in M, W^{s}(p)$ and $W^{u}(q)$ have transversal intersection.

This is proved in [55] and [114]. In [86] there is a polished version, which also proves the noncompact case. In [2] there is an account done in the general framework of transversality theory.

Note that if $f$ satisfies (6.7) (a) then for each $m \in Z^{+}$, the number of periodic points of period $m$ is finite, i.e., $N_{m}<\infty$.

The other main approximation theorem is related to Pugh's $C^{1}$ solution of the problem of the "closing lemma" [91]. This can be stated as follows.

(6.8) Theorem (Pugh). Suppose $f \in \operatorname{Diff}(M)$, and $x \in M$ is recurrent in the sense that $\phi: Z \rightarrow M$ defined by $\phi(m)=f^{m}(x)$ is not a homeomorphism onto its image. Then there is a $C^{1}$ approximation $f^{\prime}$ of $f$ such that $x$ becomes a periodic point of $f^{\prime}$.

Pugh uses the methods of (6.8) to prove the following [92].

(6.9) TheOREM. For compact $M$, the property (6.1)(b) is generic in the $C^{1}$ sense. In other words suppose we put the $C^{1}$ topology on $\operatorname{Diff}(M)$ and let $G$ be the set of $f \in \operatorname{Diff}(M)$ with the property that the periodic points are dense in $\Omega(f)$. Then $G$ is a Baire set.

Unfortunately the $C^{r}$ analogues for $r>1$ of (6.8) and (6.9) are yet unproved. Furthermore, in my opinion, it is important to find "conceptual" proofs of Pugh's important results.

We end by stating the three basic problems raised here.

(6.10) Problems. (a) Approximation problem: For compact $M$, approximate $\left(C^{r}\right.$, large $r$ preferably) any $f \in \operatorname{Diff}(M)$ by $f^{\prime}$ satisfying Axiom $\mathrm{A}$ and Axiom B. In this perhaps the most important property is Axiom $\mathrm{A}(\mathrm{a})$.

(b) Find all (in some sense) possible indecomposable hyperbolic sets of nonwandering points up to topological conjugacy. This includes, as a special case, find all Anosov diffeomorphisms (such that $\Omega=M$ ).

(c) Find the possible ways of fitting the $W^{s}\left(\Omega_{i}\right)$ together to define $f: M \rightarrow M$ as in (6.3) and (6.5). This is sort of a generalized Morse theory type problem and essentially problem (6.6)a. 
It could happen that (6.10)(a) has a negative answer. This would add difficulty to the conjugacy problem! One would proceed by adding the corresponding counterexamples to those of this paper and enlarge the unifying framework.

On the other hand an affirmative answer to (6.10) (a) would imply that this survey gives the basic framework to the conjugacy problem and that answering (6.10)(b), (c) would be filling in the body.

I.7. Our goal in this section is to give at least a full sketch of proofs of the spectral decomposition theorem (6.2) and its corollary. In doing so we state a general stable manifold theorem and use it to show the existence of canonical coordinates on our hyperbolic sets. We first give some preliminary lemmas.

(7.1) Lemma. (a) If $p$ is a periodic point of $f \in \operatorname{Diff}(M)$, and $U$ is an open set in $M$ such that $U \cap W^{s}(p) \neq \varnothing$, then the closure of $\bigcup_{m>0} f^{m}(U)$ $\supset W^{u}(p)$.

(b) Furthermore, if $q$ is a second periodic point and $W^{u}(p) \cap W^{s}(q)$ contains a point of transversal intersection, then $\bigcup_{m>0} f^{m}(U) \cap W^{s}(q) \neq \varnothing$.

Proof. Note first that by replacing $f$ by a power of $f$, we may as well assume $p$ and $q$ are fixed points to begin with. Since $f: W^{u}(p)$ $\rightarrow W^{u}(p)$ is an expansion, it follows that if a neighborhood of $p$ in $W^{u}(p)$ is in the closure of $U_{m>0} f^{m}(U)$, then so is all of $W^{u}(p)$. Thus we see that (a) is transformed into a local problem about a neighborhood $N$ of $p$ by replacing $U$ by $f^{k}(U) \cap N$ for some large $n$.

In case $f$ is linear in some chart about $p$ the conclusion of (a) is easily checked directly, and finally the general case can be reduced to this one by an appeal to Hartman's (and Grobman's [74]) theorem [39], which gives a local topological equivalence to the linear case.

The second part of (7.1) can be proved with little trouble by using the linear Lemma 5.2 of [115] or one can use again Hartman's theorem and a topological intersection argument. The reduction to the local case is again clear. A stronger lemma than this, the " $\lambda$-lemma" is in [82].

(7.2) Lemma. Let $f: M \rightarrow M$ be a diffeomorphism with hyperbolic periodic points $p_{i}, i=0, \cdots, n$ such that $p_{0}=p_{n}$. Suppose for each $i=0, \cdots, n-1, x_{i} \in W^{u}\left(p_{i}\right) \cap W^{s}\left(p_{i+1}\right)$ is a point of transversal intersection. Then each $x_{i}$ is nonwandering.

Proof. Let $x_{i}$ for some $i$ be as in (7.2) and $U$ be a neighborhood of $x_{i}$ in $M$. Then $\left(\cup_{m>0} f^{m}(U)\right) \cap W^{s}\left(p_{j}\right) \neq \varnothing$ every $j$ using (7.1) inductively. By (7.1)(a) Closure $U_{m>0} f^{m}(U) \supset W^{u}\left(p_{i}\right)$. This shows $x_{i}$ is nonwandering. 
We next come to the general stable manifold theory which we put into the following form.

(7.3) Generalized Stable Manifold Theorem. Suppose $\Lambda \subset M$ is a hyperbolic set of $f \in \operatorname{Diff}(M)$ (that is, $\Lambda$ is compact, invariant with the usual splitting of $T_{\Lambda}(M)$, see §I.6) with some metric on $M$. Then for each $x \in \Lambda$, there is an injective immersion $J_{x}^{s}=J_{x}: W^{s}(x) \rightarrow M$ with the following properties:

(a) $x \in J_{x}\left(W^{s}(x)\right)$, and $y \in J_{x}\left(W^{s}(x)\right)$ if and only if $d\left(f^{m}(x), f^{m}(y)\right) \rightarrow 0$ as $m \rightarrow \infty$.

(b) $f\left(J_{x}\left(W^{s}(x)\right)=J K_{f(x)} W^{s}(f(x))\right.$. Let $J_{x}\left(W^{s}(x)\right)=W^{s}(x)$ now.

(c) $\cup_{x \in \Lambda}\left(W^{s}(x)\right)=\left\{y \in M \mid f^{m}(y) \rightarrow \Lambda, m \rightarrow \infty\right\}$.

(d) For $x, y \in \Lambda, W^{s}(x)$ and $W^{s}(y)$ either coincide or are disjoint.

(e) The tangent space of $W^{s}(x)$ at $y$ is $E_{y}^{s}$ for each $y \in \Lambda$ (here $E_{y}^{s}$ is part of the data of the hyperbolic splitting).

(f) $W^{s}(x)$ and $W^{s}(y)$ are $C^{1}$ close on compact sets for $x, y \in \Lambda$ close.

For $\Lambda$ a point this is the stable manifold theorem for a fixed point, §I.2. In (7.3), $W^{s}(x)$ for $x \in \Lambda$ is called the stable manifold of $x$. The unstable manifold $W^{u}(x)$ is defined as the stable manifold of $f^{-1}$ at $x$.

We will try now to give the history and background of (7.3). Of course it all starts with $\Lambda$ a point from Poincaré, Perron, etc. as in \$I.2. Anosov, using the basic work of Perron, proved (7.3) in the case $\Lambda$ is all of $M$. This is the way he proved the structural stability in \$I.3. Seeing the need for a more general version of stable manifold theory, because of Axiom A, I asked I. Kupka if he could give such a proof. In substance at least, he proved the above (7.3). All of the proofs in stable manifold theory, however, have been unsatisfactory from a conceptual point of view. On the other hand, at this writing it appears that the situation has been remedied by M. Hirsch. He seems to have a fully satisfactory proof of the above (7.3).

Added in proof. C. Pugh has a good proof of (7.3).

From the stable manifold theory we now construct what we call canonical coordinates on $\Omega(f)$ where $f \in \operatorname{Diff}(M)$ satisfies Axiom A. If $W^{s}(x)$ is as in (7.3), then we will denote an $\epsilon$ neighborhood of $x$ in $W^{s}(x)$ in the intrinsic (metric) topology by $W^{s}(x, \epsilon)$. Then let $\breve{W}^{s}(x, \epsilon)$ be the set $W^{s}(x, \epsilon) \cap \Omega$ etc.

(7.4) Theorem (Existence of Canonical Coordinates). Suppose $f \in \operatorname{Diff}(M)$ satisfies Axiom A and that $x \in \Omega=\Omega(f)$. Then there is $\epsilon>0$, independent of $x$, and a canonical map $I_{x}: V \rightarrow M$ where $V$ is a neighborhood of $x \times x$ in $\widehat{W}^{u}(x) \times W^{s}(x)$, which is a homeomorphism of $V$ onto a neighborhood of $x$ in $\Omega$. On $W^{u}(x, \epsilon) \times x, I_{x}$ is the inclusion $J_{x}^{u}$ and on $x \times W^{s}(x, \epsilon), I_{x}$ is the inclusion $J_{x}^{s}$. The map $I_{x}$ is defined at $(p, q) \in V \subset \widetilde{W}^{u}(x, \epsilon) \times \mathscr{W}^{s}(x, \epsilon)$ as the unique intersection of $W^{s}(p, \epsilon)$ and $W^{u}(q, \epsilon)$ in $M$. 
Proof. This follows directly from a systematic application of (7.3) and (7.2). The map is well defined into $M$ by (7.3). The image of $I_{x}$ is in $\Omega$ by (7.2) and the fact that the periodic points are dense in $\Omega$. In a similar way, one checks that a neighborhood of $x$ in $\Omega$ is in the image of $I_{x}$. The injectivity of $I_{x}$ is a consequence of the stable manifold theorem, that the $W^{s}(p)$ for different $p$, either coincide or are disjoint.

Moving toward the proof of (6.2) we give first the following lemma:

(7.5) Lemma. Suppose $f \in \operatorname{Diff}(M)$ satisfies Axiom A, $\Omega=\Omega(f)$ the nonwandering points. Given $x \in \Omega$, suppose $N$ is a neighborhood of $x$ in $\Omega$ with the local product structure of (7.4). If $U$ is any nonempty open subset of $N$, then $U_{m \geq 0} f^{m} U$ and $U_{m \leqq 0} f^{m}(U)$ each contain a dense subset of $N$.

Proof. It is sufficient to consider just one of the two cases. Let $q$ be a periodic point of $U$ with stable and unstable manifolds $W^{s}(q)$ and $W^{u}(q)$. There exist such $q$ since the periodic points are dense in $\Omega$ by Axiom A. Now let $p$ be an arbitrary periodic point of $N$. There are points of transversal intersection $x \in W^{u}(p) \cap W^{s}(q), \quad x^{\prime} \in W^{u}(q)$ $\cap W^{s}(p)$, with $x, x^{\prime}$ in $\Omega$. Then $x \in U_{m \leq 0} f^{m} U$, and so $p$ is in the closure of $\bigcup_{m \leq 0} f^{m} U$. Since the periodic points are dense in $N$, this proves (7.5).

We now prove (6.2).

For $x \in \Omega$, let $N=N(x)$ be given as in the previous lemma and define $\Omega_{x}=$ Closure $\bigcup_{m \in Z} f^{m}(N)$. From the previous lemma it follows that $\Omega_{x}$ does not depend on any choices. In fact, it follows equally well from (7.5) that for $x, y \in \Omega$, either $\Omega_{x}$ and $\Omega_{y}$ coincide or are disjoint. Furthermore the union $\Omega=U_{x} \Omega_{x}$ is actually a finite union and all of the properties of (6.2) are checked very easily now using the previous lemma. Note that one obtains directly that any open set in $\Omega_{i}$ has a dense orbit (i.e., $U_{m} f^{m}(U)$ is dense). From Birkhoff [15] one then obtains topological transitivity.

We show how (6.3) follows from (6.2).

For each $x \in M, m \rightarrow \infty: f^{m} x \rightarrow \Omega$ from the definition of nonwandering. Given $x \in M$, we claim there is a unique $i$ such that $f^{m} x \rightarrow \Omega_{i}$ as $m \rightarrow \infty$. For each $i=1, \cdots, k$, choose open sets $V_{i}, U_{i}$ such that $V_{i} \supset U_{i} \supset \Omega_{i}, V_{i}$ disjoint and $f^{-1} U_{i} \cup f U_{i} \subset V_{i}$. Now, given $x \in M$, suppose there exist $k, l$ such that $\Omega_{k} \cap \lim _{n \rightarrow \infty} f^{n} x \neq \varnothing, \Omega_{l} \cap \lim _{n \rightarrow \infty} f^{n} x$ $\neq \varnothing, k \neq l$. Then there exist for each $j=1,2, \cdots$ positive integers $m_{j}, l_{j}$ such that $f^{m_{i}} \in U_{k}, f^{m_{j}+l_{j} \in U_{l}}$ where $m_{j}<m_{j}+l_{j}<m_{j+1}$ for each $j$. Then there exists $n_{j}, m_{j}<n_{j}<m_{j}+l_{j}$ such that $f^{n j} x \notin \bigcup_{i=1}^{k} U_{i}$, for every $j$. Therefore $\lim _{j \rightarrow \infty} \mid f^{n_{j}} x \in \Omega$, which is impossible. Thus $k=l$ and our assertion is proved, which in turn yields (6.3). 
Note finally that the following proposition is clear from the previous material in this section.

(7.6) Proposition. Let $\Omega_{i}$ be as in (6.2). (a) Then for any $x \in \Omega_{i}$, $W^{s}(x)$ and $W^{u}(x)$ each contain a dense set of $\Omega_{i}$.

(b) In particular if $f: M \rightarrow M$ is an Anosov diffeomorphism of a compact manifold with $\Omega=M$, then every stable manifold is dense in $M$.

(c) $W^{u}\left(\Omega_{i}\right) \cap W^{s}\left(\Omega_{i}\right)=\Omega_{i}$.

One obtains (a) from the topological transitivity and the local product structure on $\Omega_{i}$. (b) follows from (a). One checks (c) by first showing that $W^{u}\left(\Omega_{i}\right) \cap W^{s}\left(\Omega_{i}\right) \subset \Omega$ using (7.2) and the fact that the periodic points are dense in $\Omega$. Then apply (6.2).

I.8. The goal of this section is to sketch the proofs of Theorems (6.5) and (6.6). We begin by introducing the generally useful notion of a filtration of a diffeomorphism.

A filtration then of $f \in \operatorname{Diff}(M), M$ compact, is a sequence of closed submanifolds, $M=M_{0} \supset M_{1} \supset M_{2} \supset \cdots \supset M_{k}=\varnothing$ where each $M$ is an open subset together with its smooth boundary and $f\left(M_{i}\right) \subset$ interior of $M_{i}$.

(8.1) Proposition. If $\left\{M_{i}\right\}$ is a filtration for $f \in \operatorname{Diff}(M)$, then it is also a filtration for a $C^{0}$ approximation of $f$. Furthermore $\Omega(f) \cap \partial M_{i}=\varnothing$ for each $i$.

This is easily checked. If $\left\{M_{i}\right\}$ is a filtration for $f \in \operatorname{Diff}(M)$ then we can decompose $\Omega(f)=\Omega_{1} \cup \ldots \cup \Omega_{k-1}, \Omega_{i}$ compact invariant, by defining $\Omega_{i}=\Omega \cap\left(M_{i}-M_{i-1}\right)$. We will call this the $\Omega$-decomposition of the filtration.

We assume now that $f$ satisfies Axioms $\mathrm{A}$ and $\mathrm{B}$, with $\Omega_{i}, W^{s}\left(\Omega_{i}\right)$, etc. as in $\$ I .6$.

(8.2) Proposition. If $W^{u}\left(\Omega_{i}\right) \cap W^{s}\left(\Omega_{j}\right) \neq \varnothing$, then for any $p \in \Omega_{i}$, $q \in \Omega_{j}, W^{u}(p)$ and $W^{s}(q)$ have a point of transversal intersection.

PRoOF. This is a consequence of Axiom B and §I.7. Then by (7.1) we obtain

(8.3) Corollary. If $W^{u}\left(\Omega_{i}\right) \cap W^{s}\left(\Omega_{j}\right) \neq \varnothing$, then $W^{u}\left(\Omega_{j}\right) \subset$ Closure $W^{u}\left(\Omega_{i}\right)$.

(8.4) Proposition. If $W^{u}\left(\Omega_{i}\right) \cap W^{s}\left(\Omega_{i+1}\right) \neq \varnothing, i=0, \cdots, m-1$ and $\Omega_{0}=\Omega_{m}$, then all the $\Omega_{i}$ coincide.

PRoOF. Let periodic points $p_{i} \in \Omega_{i}$ for each $i$. Then by (8.2), $W^{u}\left(p_{i}\right)$ and $W^{s}\left(p_{i+1}\right)$ have a point of transversal intersection $q_{i}$ for each $i$. Apply (7.2) to see that each $q_{i} \in \Omega$. But then $q_{i} \in \Omega_{i} \cap \Omega_{i+1}$, so indeed the $\Omega_{i}$ coincide.

From (8.3) and (8.4) follows 
(8.5) Proposition. The relation $\leqq$ defined in $\$ 1.6$ is a partial ordering.

REMARK. One could define $\leqq$ in these alternate ways as long as Axiom $\mathrm{B}$ was modified accordingly and the whole theory would be the same.

Alternate way $1 . \Omega_{i} \leqq \Omega_{j}$ if $\operatorname{Clos} W^{u}\left(\Omega_{j}\right) \cap \operatorname{Clos} W^{s}\left(\Omega_{i}\right) \neq \varnothing$.

Alternate way 2. $\Omega_{i} \leqq \Omega_{j}$ if for any pair of neighborhoods $U_{i}$ of $\Omega_{i}$, $U_{j}$ of $\Omega_{j}$ there is $x \in U_{j}, m \in Z^{+}$such that $f^{m}(x) \in U_{i}$.

(8.6) Proposition. Suppose $f \in \operatorname{Diff}(M)$ satisfies Axioms A and B, with $\Omega_{i}$ as in the spectral decomposition theorem. Then there exists a filtration of $M, M=M_{0} \supset M_{1} \supset \cdots \supset M_{k}=\varnothing$ where $\Omega \cap M_{j}-M_{j-1}$ is precisely one of the $\Omega_{j}$ (re-indexing if necessary, so $\Omega_{j}=\Omega \cap M_{j}-M_{j-1}$ ) of the spectral decomposition. Similarly one has a filtration for $f^{-1}$, $M=M_{0}^{\prime} \supset M_{1}^{\prime} \supset \cdots \supset M_{k}^{\prime}=\varnothing$. Given neighborhoods $U_{i}$ of $\Omega_{i}$, one may choose the filtrations so that $U_{i}$ contains $\left(M_{i}-M_{i-1}\right) \cap\left(M_{i}^{\prime}-M_{i-1}^{\prime}\right)$ for each $i$. Consequently perturbations $f^{\prime}$ of $f$ will satisfy $\Omega\left(f^{\prime}\right) \subset \cup U_{i}$.

Proof of (8.6). One uses the diagram. Start by taking $M_{k}=\varnothing$, $M_{k-1}$ a neighborhood of an extreme vertex (attractor), $M_{k-2}=M_{k-1} \cup V$ where $V$ is a contracting neighborhood of a second attractor, continuing until all the attractors are used up, obtaining $M_{l} \supset M_{l-1}$ $\supset \cdots \supset M_{k}$ say. Choose $\Omega_{j}$ so that $\Omega_{j}$ is next to only attractors in the diagram. One defines $M_{l-1}$ as $M_{l} \cup W$ where $W$ is of the form $\bigcup_{m_{0}>m>0} f^{m}\left(W_{0}\right), m_{0}$ some large integer and $W_{0}$ is a small neighborhood of $\Omega_{j}$. One may think of $W$ as a neighborhood of $W^{u}\left(\Omega_{j}\right)$. By continuing in this way one obtains the desired filtrations.

Conversations with $M$. Shub were very useful in the following.

To prove $\Omega$-stability for $f \in \operatorname{Diff}(M)$ satisfying Axioms $\mathrm{A}$ and $\mathrm{B}$, one generalizes the procedure of Moser in the Appendix written by Mather. Instead of the map $A$ defined there one uses the map

$$
B: \operatorname{Diff}(M) \times C^{0}(\Lambda, M) \rightarrow C^{0}(\Lambda, M)
$$

defined by $B(g, h)=g h f^{-1}$. Here $C^{0}(\Lambda, M)$ is the space of continuous maps of $\Lambda$ into $M$ with the uniform topology with $\Lambda=\Omega_{i}$ an indecomposable piece of $\Omega(f) . C^{0}(\Lambda, M)$ is a manifold and $B$ has its second partial derivative continuous in both variables. A version of the implicit function theorem yields a continuous map

$h: \Lambda \longrightarrow M$ such that

$$
\begin{aligned}
& \Lambda \stackrel{h}{\longrightarrow} M \\
& \downarrow f \quad \downarrow g \\
& \Lambda \stackrel{h}{\longrightarrow} M \quad \text { commutes }
\end{aligned}
$$


(providing $g$ is close enough to $f$ in $C^{1}$ ). There remains to complete the proof of $\Omega$-stability of $f$, two things. First, $h$ is $1-1$. Here the Moser argument does not work. The proof goes by

(8.7) Proposition. $f: \Lambda \rightarrow \Lambda$ is expansive. This means there is $\epsilon>0$ such that for any $x, y \in \Lambda, x \neq y$, there is $n \in Z$ with $d\left(f^{n} x, f^{n} y\right)>\epsilon$.

The proof of this proposition follows from the fact that $\Lambda$ is a hyperbolic set for $f$. Then the fact that $h$ makes the above diagram commute and is close to the identity leads to the injectivity of $h$.

The second point to check is that when $h$ is defined on each $\Omega_{i}$ as above, $h(\Omega(f))=\Omega(g)$. Since the periodic points are dense in $\Omega(f)$ it follows that $h(\Omega(f)) \subset \Omega(g)$. Furthermore, by (8.6) we may assume that $\Omega(g) \subset$ small neighborhoods of $\Omega_{i}$. Thus the proof of $\Omega$-stability is reduced to the study of what happens in a small neighborhood of $\Omega_{i}$. The stable manifold analysis finally takes care of this last point.

To finish our program, we must show that if $g$ has been chosen close enough to $f$, then $g$ also satisfies Axioms A and B. All of this is a consequence of the above provided $\Omega(g)=h(\Omega(f))$ has a hyperbolic structure for $g$. This proceeds by showing bounded hyperbolic linear maps on Banach spaces are open using the spectral theory at the end of [96], and then using the values of sections of the Banach space splitting to reconstruct the vector bundle splitting.

I.9. On basic sets of diffeomorphisms. This section is devoted to the problem of finding all the possible $\Omega_{i}$ that could occur in the spectral decomposition theorem (6.2) for diffeomorphisms of compact manifolds satisfying Axiom A. In other words we discuss what is known about Problem (6.10)(b). Expanding on this define a basic set of $f \in \operatorname{Diff}(M)$ to be one of the $\Omega_{i}$ of (6.2) where $f$ satisfies Axiom A.

(9.1) Problem. Find all basic sets up to topological conjugacy. Do they always have a rational zeta function? Are they all locally the product of a Cantor set and a manifold? Can they be given some type of algebraic structure?

One can consider a possibly more general, but localized picture by considering $f: U \rightarrow M$ with $U$ an open set of $M, f$ a diffeomorphism on to its image with $\Lambda \subset U$ satisfying

(9.2) (a) $\Lambda$ is compact and $f(\Lambda)=\Lambda$,

(b) $\Lambda$ is a hyperbolic set for $f$ (see $\S$ I.6),

(c) the periodic points of $f$ are dense in $\Lambda$,

(d) $f$ is topologically transitive on $\Lambda$,

(e) $\bigcap_{m \in Z} f^{m}(U)=\Lambda$.

Since the basic sets have neighborhoods $U$ which satisfy (9.2) we may consider 
(9.3) Problem. Find all $\Lambda$ satisfying (9.2).

There is a construction which allows one to replace $f: U \rightarrow M$ by $g: \widetilde{U} \rightarrow \widetilde{U}$ in (9.2). On $U \times Z$ say points $(x, m)$ and $\left(x^{\prime}, n\right)$ are equivalent if $x^{\prime}=g^{n-m}(x)$. Then the quotient space $\widetilde{U}$ is a manifold (not necessarily Hausdorff) and one has a diffeomorphism $g: \tilde{U} \rightarrow \widetilde{U}$ induced by $(x, m) \rightarrow(x, m+1)$. Define $\tilde{\Lambda} \subset \tilde{U}$ as the image of $(\Lambda, 0)$ under the projection $\pi: U \times Z \rightarrow U$.

-We will say that $\Lambda$ in (9.2) is an attractor if $U$ can be chosen so that $\cap_{m>0} f^{m}(U)=\Lambda$. Then when $\Lambda$ is the basic set of a diffeomorphism satisfying Axioms $\mathrm{A}$ and $\mathrm{B}$, an attractor corresponds to a vertex lying at an extreme point of the diagram of $f$.

A special case of (9.1) and (9.3) is to find the attractors. Note that no symbolic flow of $\S I .5$ can be an attractor, but that every Anosov diffeomorphism with $\Omega=M$ is already an attractor.

We will give an outline of all the ways we know of constructing basic sets; then we will go into more detail. First consider these four groups of basic sets:

(9.4) (a) Group 0. These are characterized by dimension $\Lambda=0$.

(b) Group A. This is Anosov case with $\Omega=M$.

(c) Group DE. These are derived from expanding maps and will be described subsequently.

(d) Group DA. These are derived from Anosov diffeomorphisms and will also be described subsequently.

Furthermore one may take finite products of any of these to obtain other basic sets (see $§ I .10$ ).

Group 0 is discussed first. This includes the finite $\Lambda$ (periodic orbits) and the shift automorphisms $\Lambda_{N}$ of $\S I .5$. It seems likely to me that every basic set in group 0 is topologically conjugate to some closed invariant subset of $\Lambda_{N}$. Call $\Lambda \subset \Lambda_{N}$ a subshift if $\Lambda$ is closed, invariant, and the periodic points are dense in $\Lambda$. One can ask generally to what extent the subshifts occur as basic sets.

The following construction may shed some light on the above imbedding problem. Suppose $\Lambda$ is a basic set of dimension 0 relative to $f: U \rightarrow M, U_{1} \cup \ldots \cup U_{N}$ a disjoint union of local product neighborhoods of (7.4) which cover $\Lambda$ (such $U_{i}$ can always be found). Then let $g: \Lambda_{N} \rightarrow \Lambda_{N}$ be the shift automorphism on the $N$-symbols $U_{1}, \cdots, U_{N}$ and define $\alpha: \Lambda \rightarrow \Lambda_{N}$ by $\alpha(x)(m)=U_{i}$ where $x \in \Lambda, m \in Z$, and $f^{m}(x) \in U_{i}$. Then it is easily checked that $\alpha$ is continuous and equivariant. Can the $U_{i}$ be chosen so that $\alpha$ is injective?

The following is a nontrivial example of a subshift as a basic set. We describe it in the following figure as a diffeomorphism of a 2-disk 
into itself which can be extended to a diffeomorphism $S^{2} \rightarrow S^{2}$ by adding an expanding disk to the original.

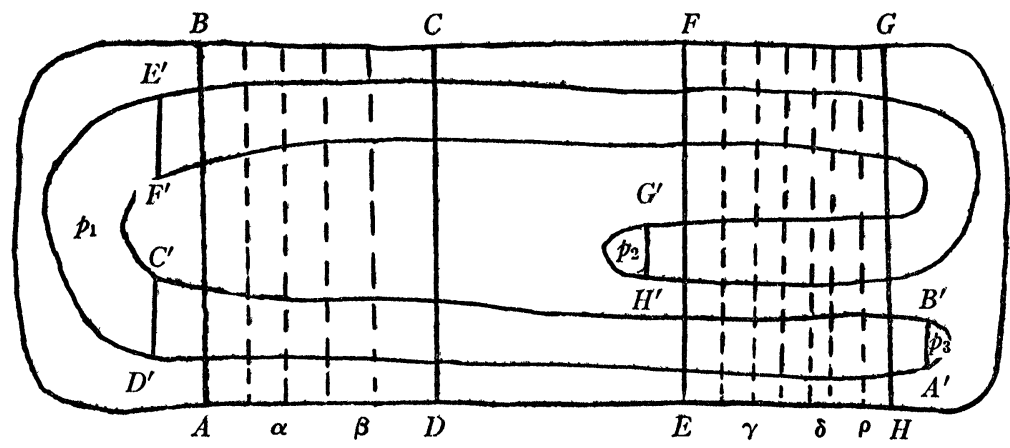

The construction follows in the pattern of those in $\$ 1.5$. The diagram is simply given by $\&$ where the top vertex is an expanding fixed point and the bottom vertex corresponds to the periodic orbit consisting of $p_{1}, p_{2}$ and $p_{3}$. The reader can check that the middle vertex of the diagram corresponds to a subshift $\Lambda$ of the shift on five symbols. In fact if $\Lambda_{b}$ is the shift space on the symbols $\alpha, \beta, \gamma, \delta, \rho$ corresponding to the indicated columns in the figure, then $\Lambda$ consists of bi-infinite sequences which do not carry any of the following combinations $\beta \gamma, \beta \delta, \beta \rho$, $\alpha \alpha, \alpha \beta, \gamma \delta, \gamma \gamma, \gamma \rho, \delta \alpha, \delta \beta, \rho \alpha, \rho \beta$.

Generally speaking, relative to a shift automorphism a block is a finite sequence of symbols, e.g., $\beta$, etc. in the previous sentence. A subshift is said to be of finite type if it is of the form, all sequences which do not contain a certain finite set of blocks. Thus the above $\Lambda$ is of finite type.

Related to the previous problems on basic sets of dimension 0 are the following theorems:

(9.5) THEOREM (O. LANFORD). Every subshift of finite type has a rational zeta function.

(9.6) THEOREM (R. Bowen). There exist subshifts with irrational zeta functions.

In fact Lanford has improved Bowen's theorem to show that most subshifts have irrational zeta functions.

We don't go beyond the discussion of $\$ 1.3$ on the Anosov case except to remark that it seems probable that if a basic set is a submanifold then the restriction of the diffeomorphism is conjugate to an Anosov diffeomorphism. 
J. Moser has shown me an example of a basic set which is a submanifold but not a $C^{1}$ submanifold.

For the DE group we use the examples of Shub [108] of expanding endomorphisms of compact manifolds-see $\$ I .10$. For each expanding endomorphism, we will construct a basic set which is an attractor. This goes as follows.

Suppose then $f: M \rightarrow M$ is an expanding endomorphism of a compact manifold. Let $D$ be the unit disk of dimension one larger than the dimension of $M$, with $M$ imbedded in $D \times M$ as $0 \times M$. Let $\lambda$ satisfy $0<\lambda<1$ and define $g_{\lambda}: D \times M \rightarrow D \times M$ by $g_{\lambda}(x, y)=(\lambda x, y)$. Next let $\phi: 0 \times M \rightarrow D \times M$ be a $C^{1}$ approximation of the map $0 \times M$ $\rightarrow D \times M,(0, y) \rightarrow(0, f(y))$ such that $\phi$ is an embedding. This is possible by dimensional reasons (the Whitney imbedding theorem). Let $T$ be a tubular neighborhood of $\phi(M)$ with fibers being the various components of $T \cap(D \times y), y \in M$. Now extend $\phi$ to $\psi: D \times M \rightarrow T$ in a fiber preserving way so that $\psi$ is even a diffeomorphism. Our desired map $D \times M \rightarrow D \times M$ is then the composition $\psi g_{\lambda}=h$ for $\lambda$ small enough. It can be checked that for sufficiently small $\lambda$, the set $\Lambda=\bigcap_{m>0} h^{m}(D \times M)$ has a hyperbolic structure and is in fact a basic set. It is locally the product of a Cantor set and a manifold whose dimension is that of $M$.

The following figure gives $D \times M$ and its image under $h$ when the starting point is the expanding endomorphism of $S^{1} \rightarrow S^{1}$ defined by $z \rightarrow z^{2}$.

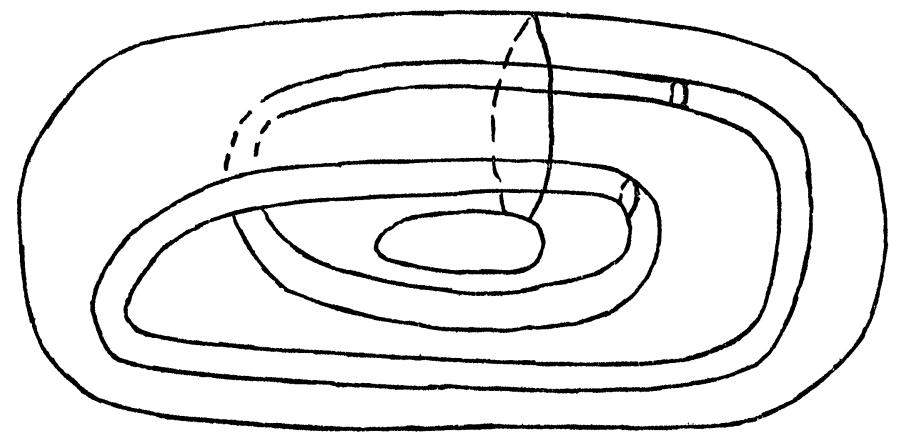

Finally we show how the DA group (9.4d) goes by giving the first case using an extended type of surgery on the Anosov diffeomorphism of the 2-torus.

One changes the toral diffeomorphism on a small "square" neighborhood $Q$ of the fixed point corresponding to $(0,0)$ in $R^{2}$. Initially we have the square $Q=A B C D$ linearly mapped into $A^{\prime} B^{\prime} C^{\prime} D^{\prime}$ as in the following figure. 


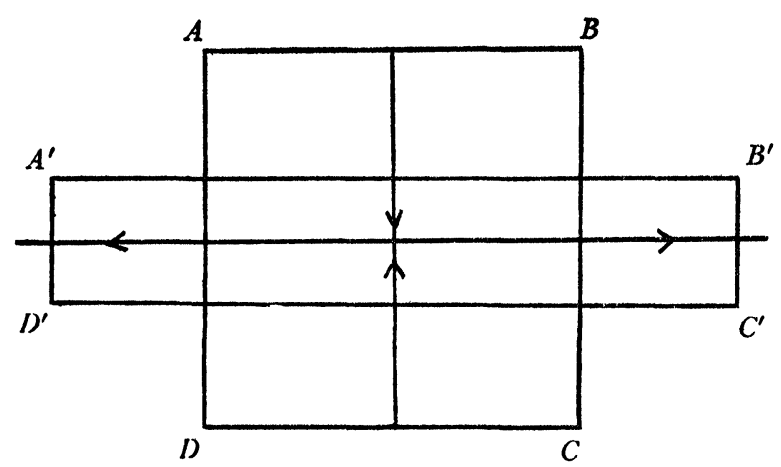

Without changing the diffeomorphism outside a neighborhood of the boundary of $Q$, we can change $f$ on $Q$ so that we have three fixed points in $Q$ as illustrated in the following figure.

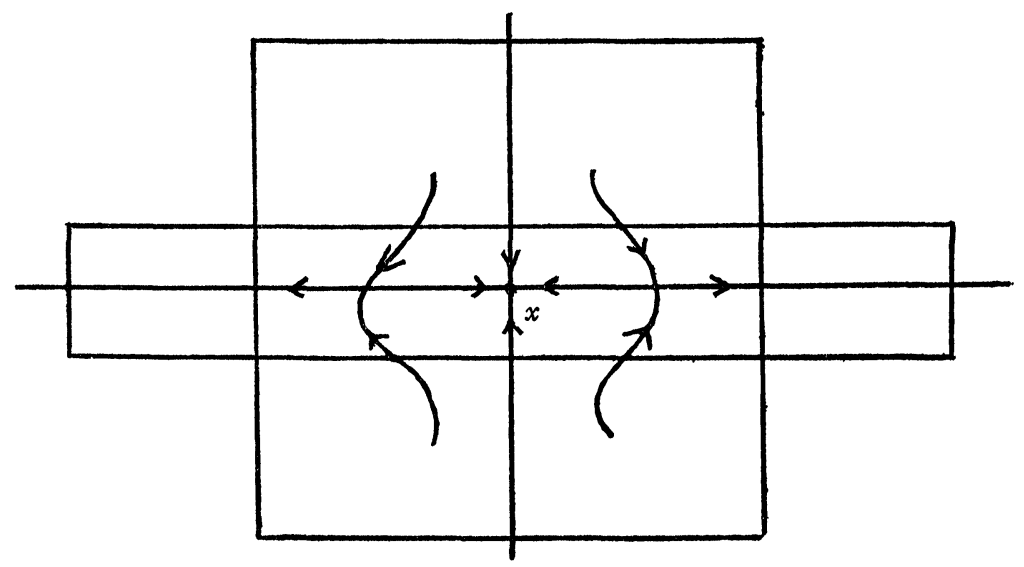

Now $T^{2}$ can be written as the union of a single two dimensional stable manifold of the fixed point $x, W^{s}(x)$ and a one dimensional basic $\Lambda$. We leave the (many) details to the reader.

One can apply this construction to any Anosov diffeomorphism.

As this was written, we received a very interesting manuscript of R. Williams [127] on 1-dimensional basic sets, which certainly appears to extend some of the above results.

I.10. Final remarks on conjugacy problems. We cover briefly a number of final miscellaneous points related to the diffeomorphism problem of part I. The first question is: what role do products play? 
(10.1) Proposition. Let $\Omega_{i}$ be the set of nonwandering points of $f_{i} \in \operatorname{Diff}\left(M_{i}\right), i=1,2$. Then the set of nonwandering points of the product $f_{1} \times f_{2} \in \operatorname{Diff}\left(M_{1} \times M_{2}\right)$ is contained in $\Omega_{1} \times \Omega_{2}$. Furthermore if the periodic points are dense in $\Omega_{1}$ and $\Omega_{2}$, then $\Omega=\Omega_{1} \times \Omega_{2}$.

This is checked easily from the definitions.

(10.2) Proposition. If $f_{i} \in \operatorname{Diff}\left(M_{i}\right), i=1,2$ satisfying (2.2), then so does the product $f_{1} \times f_{2} \in \operatorname{Diff}\left(M_{1} \times M_{2}\right)$.

This follows from (10.1) and the fact that $W^{s}(p, q)=W^{s}(p) \times W^{s}(q)$. Furthermore one sees from the definitions that

(10.3) Proposition. The product of two Anosov diffeomorphisms is an Anosov diffeomorphism.

The last two propositions are essentially contained in (equally easily checked)

(10.4) Proposition. If $f_{i} \in \operatorname{Diff}\left(M_{i}\right), i=1,2$, both satisfy Axioms $\mathrm{A}$ and $\mathrm{B}$, then so does the product $f_{1} \times f_{2}$. Furthermore so does $f_{1}^{m}, m \in Z$, $m \neq 0$.

Thus if $f_{1} \in \operatorname{Diff}\left(M_{1}\right)$ is an Anosov diffeomorphism and if $f_{2} \in \operatorname{Diff}\left(M_{2}\right)$ satisfies (2.2), then the product $f_{1} \times f_{2}$ is $\Omega$-stable (6.5). This product, however, is not structurally stable. Moreover, there is an open set $U$, in general, in $\operatorname{Diff}\left(M_{1} \times M_{2}\right)$ near $f$ with the property that $U$ contains no structurally stable diffeomorphisms. This is described in [116]. It is the example mentioned in $\$ 1.1$ to show that one had to weaken the concept of structural stability to get a successful theory. There is also an exposition of this fact in [11] and a further variant in [87].

We now define a modification of diffeomorphisms related to the notion of "surgery" in differential topology.

Suppose $f \in \operatorname{Diff}(M)$ has the property that there is a compact submanifold with boundary $M_{1}, \operatorname{dim} M=\operatorname{dim} M_{1}$, such that $f\left(M_{1}\right)$ is contained in the interior of $M_{1}$. Then it follows that $\Omega=\Omega(f)$ is equal to (interior $\left.M_{1}\right) \cap \Omega \cup\left(M-M_{1}\right) \cap \Omega=\Omega_{1} \cup \Omega_{2}$ where each $\Omega_{i}$ is compact and invariant. Furthermore, a similar decomposition can be done even for any sufficiently good $C^{0}$ approximation of $f$. This is a special case of the filtrations of $\S I .8$.

For surgery, in addition to the above $f$ suppose that $g: V \rightarrow V$ is a diffeomorphism of a compact manifold with boundary into its interior. Suppose further that there is a diffeomorphism $h: \mathrm{Cl}\left(M_{1}-f\left(M_{1}\right)\right)$ $\rightarrow \mathrm{Cl}(V-g(V))$ with $g h=h f$. An isotopy condition on $f, g$ is sufficient 
to guarantee the existence of $h$ at least in case Closure $(V-f(V))$ $=1 \times V$. Then one may replace $M_{1}$ by $V$ and redefine $f$ on $M_{1}$ by $g$ on $V$ to define $f^{\prime}: M^{\prime} \rightarrow M^{\prime}, M^{\prime}$ the modified manifold. This turns out to be a useful construction. One checks immediately that $\zeta_{f^{\prime}}$ $=\zeta_{f} \circ \zeta_{\theta} \circ \zeta_{\bar{f}}^{-1}$ where $\bar{f}$ is $f$ restricted to $M_{i}$.

A topological version of the ergodic theoretic concept, entropy, has been defined in [3]. In this paper, the authors showed that this topological entropy is positive for some of the examples we described in $\S \S I .3$ and 5 . The following problem seems natural.

(10.5) Problem. If $\Omega_{i}$ is a basic set of a diffeomorphism satisfying Axiom A (as described in the spectral decomposition theorem (6.2)), is the topological entropy of $\Omega_{i}$ positive?

As J. Palis pointed out to me, any Anosov diffeomorphism will satisfy Axioms A and B. It is possible that applying some of the subsequent theorems, one could obtain an attack on the problem: For an Anosov diffeomorphism $f \in \operatorname{Diff}(M), M$ compact, must $\Omega(f)=M$ ? (See 3.4).

Up to now we have been investigating the dynamical system generated by a single $f \in \operatorname{Diff}(M)$. One can generalize this situation to a differentiable map (or endomorphism) $f: M \rightarrow M$ (without necessarily having an inverse). This $f$ is not the generator of a group acting on $M$, but a semigroup $Z^{+}$acting on $M$. M. Shub [108] has studied this problem and found that some of the previous results extend to cover this case and some new features are found here. We state some of these now.

The simplest new problem coming up in this context is the endomorphism of the complex numbers of absolute value one, $f: S^{1} \rightarrow S^{1}$ defined by $f(z)=z^{n}, n \in Z, n>1$. Is $f$ structurally stable? As for diffeomorphisms, an endomorphism $f: M \rightarrow M$ is structurally stable if $C^{1}$ perturbations are conjugate to $f$ by a homeomorphism. Shub gives an affirmative answer in the following more general proposition [108].

(10.6) Proposition. Suppose $f: S^{1} \rightarrow S^{1}$ is $C^{1}$ with derivative everywhere $>1$. Then $f$ is conjugate by a homeomorphism to $z \rightarrow z^{n}$ where $n=$ degree $f$.

The general questions on endomorphisms of $S^{1}$ are not yet very well understood. On the other hand Shub has found a satisfactory generalization of (10.6) as follows.

Say that an endomorphism $f: M \rightarrow M$ of a complete Riemannian manifold is expanding if for each $v \in T_{x}(M),\left\|D f^{m}(x)(v)\right\| \geqq c \lambda^{m}\|v\|$, $m \in Z^{+}, c>0, \lambda>1$ independent of $v, x$. Examples of expanding endomorphisms are, of course, the circle map $z \rightarrow z^{n}$ as well as various products of these on tori. 
(10.7) Theorem (Shub [108]). Any two homotopic expanding endomorphisms of a compact Riemannian manifold are topologically conjugate.

(10.8) Corollary. Any expanding endomorphism of a compact Riemannian manifold is structurally stable.

In view of (10.7), the following becomes a reasonable problem.

(10.9) Problem. Find all expanding endomorphisms of manifolds (up to conjugacy). Also, is (10.7) true for Anosov diffeomorphisms of compact manifolds?

Shub proves for expanding endomorphisms that the manifold is covered by Euclidean space, and has produced, besides those on tori, examples on the Klein bottle and nilmanifolds.

Presumably, eventually a systematic approach will include the Anosov diffeomorphisms and Shub expanding endomorphisms. The unifying definition is, in fact, obvious.

For hyperbolic fixed points of an endomorphism $f: M \rightarrow M$, Shub defines stable and unstable manifolds, generalizing those for a diffeomorphism. In this case, however, $W^{s}$ is no longer the image of a cell, but can be any manifold (i.e., map $M$ into a point $p_{0} \in M$ and take any small perturbation. Then the stable manifold of the fixed point will be $M$ ). On the other hand $W^{u}$ is the image of a cell, but not under an immersion or a 1-1 map.

Shub generalizes the approximation theorem (6.7) to endomorphisms.

Previously, holomorphic endomorphisms of the Riemann sphere had been studied by G. Julia ([50], his "prize memoir"). Stein and Ulam [119] have made a study of certain polynomial endomorphisms of the plane using computing machines.

An extremely interesting problem is the study of maps of finite dimensional manifolds into Diff $(M)$. What are generic properties of such maps? This is called bifurcation theory. The most important work on this subject is that of J. Sotomayor [118]. He considers maps of an interval into the space of flows on 2-manifolds, and obtains a pretty complete picture in this case.

\section{APPENDIX TO PART I: ANOSOV DIFFEOMORPHISMS}

\section{BY JOHN MATHER}

In this Appendix, I give an exposition of Moser's proof that Anosov diffeomorphisms are structurally stable. (See Theorem 3.3 of §I.3.) 
The main novelty in my presentation is the use of the language of manifolds of mappings, which seems to result in conceptual simplification. I would like to thank R. Abraham for suggesting that Moser's proof might be simply expressed in the language of manifolds of mappings.

We let $M$ be a compact $C^{\infty}$ manifold and $f: M \rightarrow M$ a $C^{1}$ diffeomorphism. We let $D$ denote the topological space of diffeomorphisms of $M$ into itself with the $C^{1}$ topology, $H$ the topological space of homeomorphisms of $M$ into itself with the $C^{0}$ topology (compact open topology), and $C$ the $C^{\infty}$ Banach manifold of continuous mappings of $M$ into itself, where the topology is the $C^{0}$ topology and the manifold structure is defined in a manner similar to that by which the manifold structures on sets of mappings are defined in [1] (of Appendix).

Theorem 1 (Anosov). If $f$ is an Anosov diffeomorphism, then $f$ is structurally stable. More precisely, there exists a neighborhood $U$ of the identity of $M, \mathrm{id}_{M}$, in $H$, a neighborhood $V$ of $f$ in $D$ and a continuous mapping $g \rightarrow h(g)$ of $V$ into $U$ such that for all $g \in V, h=h(g)$ is the unique solution in $U$ of the equation

$$
h g=f h .
$$

If $E$ is a vector bundle over $M$, we let $\Gamma(E)$ denote the Banachable $R$-vector space of continuous sections of $E$ over $M$, with the $C^{0}$ topology. If, further, $x \in M$, we let $E_{x}$ denote the fiber of $E$ over $x$. We let $f_{*}: \Gamma(T M) \rightarrow \Gamma(T M)$ be the continuous linear mapping given by $f_{*}(z)=D f \circ z \circ f^{-1}$. We will consider $\Gamma(T M)$ as a Banach space, with any norm which induces its topology.

Lemma 1. If $f$ is an Anosov diffeomorphism, then $f_{*}-\mathrm{id}$ is an isomorphism.

REMARK. The converse is also true, but will not be proved here.

Proof. By the hypothesis, there exists a splitting of $T(M)$ into a continuous Whitney sum $T(M)=E^{s}+E^{u}$, invariant under $D f$, such that $D f: E^{s} \rightarrow E^{s}$ is contracting and $D f: E^{u} \rightarrow E^{u}$ is expanding. Let $f_{0}=f_{*} \mid \Gamma\left(E^{s}\right)$ and $f_{\infty}=f_{*} \mid \Gamma\left(E^{u}\right)$. Then there exists $C>0,0<\lambda<1$ such that for all $m \in Z^{+}$,

$$
\left\|f_{0}^{m}\right\|<C \lambda^{m}, \quad\left\|f_{\infty}^{-m}\right\|<C \lambda^{m} .
$$

It follows that $f_{0}-$ id and $f_{\infty}^{-1}-$ id are automorphisms of $\Gamma\left(E^{s}\right)$ and $\Gamma\left(E^{u}\right)$, resp. In fact, 


$$
\begin{gathered}
\left(f_{0}-\mathrm{id}\right)^{-1}=-\sum_{j=0}^{\infty} f_{0}^{j}, \\
\left(f_{\infty}^{-1}-\mathrm{id}\right)^{-1}=-\sum_{i=0}^{\infty} \overline{f_{\infty}^{-j} .}
\end{gathered}
$$

Hence, $f_{\infty}-\mathrm{id}=-f_{\infty}\left(f_{\infty}^{-1}-\mathrm{id}\right)$ is an automorphism. The lemma follows immediately.

Let $A: D \times C \times D \rightarrow C \times C$ be given by $A\left(g_{1}, h, g_{2}\right)=\left\langle h \circ g_{1}, g_{2} \circ h\right\rangle$.

LEMMA 2. $A$ is once differentiable in its second variable and its "partial derivative,"

$$
D_{2} A: \quad D \times T C \times D \rightarrow T C \times T C
$$

is continuous in all variables. Moreover poL is an isomorphism, where

$$
L=D_{2} A \mid\left(f \times(T C)_{\mathrm{id}} \times f\right):(T C)_{\mathrm{id}} \rightarrow(T C)_{f} \times(T C)_{f}
$$

and $p$ denotes the projection of $(T C)_{f} \times(T C)_{f}$ on $(T C)_{f} \times(T C)_{f} /$ diagonal.

Proof. The first sentence follows from the methods of [1]. Also by the methods of [1], we may make the identifications $\left((T C)_{\text {id }}=\Gamma(T M)\right.$ and $(T C)_{f}=\Gamma\left(f^{*} T M\right)$. With respect to these identifications $L$ is given by

$$
z \rightarrow\langle z \circ f, D f \circ z\rangle
$$

Let $S:\left(\Gamma\left(f^{*} T M\right) \times \Gamma\left(f^{*} T M\right)\right) /$ diagonal $\rightarrow \Gamma(T M)$ be the isomorphism induced by $\langle s, t\rangle \rightarrow t o f^{-1}-s \circ f^{-1}$. Then

$$
S \circ p \circ L=f_{*}-\text { id. }
$$

Hence, the second sentence follows from Lemma 1, completing the proof of Lemma 2.

By Lemma 2 and a suitable version of the implicit function theorem, there exists a neighborhood $V_{1}$ of $f$ in $D$ and a neighborhood $U_{1}$ of $\operatorname{id}_{M}$ in $C$ such that for all $g_{1}, g_{2} \in V$ there exists a unique $h=u\left(g_{1}, g_{2}\right) \in U_{1}$ such that

$$
A\left(g_{1}, h, g_{2}\right) \in \text { diagonal }
$$

i.e.,

$$
h \circ g_{1}=g_{2} \circ h
$$

and such that $\left\langle g_{1}, g_{2}\right\rangle \rightarrow u\left(g_{1}, g_{2}\right)$ is continuous.

Let $U_{2}$ be a neighborhood of id ${ }_{M}$ in $U_{1}$ such that for all $h_{1}, h_{2} \in U_{2}$, $h_{1} \circ h_{2} \in U_{1}$ and let $V$ be a neighborhood of $f$ in $V_{1}$ such that for all $g_{1}$, $g_{2} \in V, u\left(g_{1}, g_{2}\right) \in U_{2}$. For all $g \in V$, set $h(g)=u(g, f)$ and $h^{-}(g)=u(f, g)$. 
Setting $h=h(g), h^{-}=h^{-}(g)$, we have $h g=f h$ and $h^{-} f=g h^{-}$. Hence $h^{-} h g=h^{-} f h=g h^{-} h$ and $h h^{-} f=h g h^{-}=f h h^{-}$. Since (1) has a unique solution $h \in U_{1}$ for $g_{1}, g_{2} \in V_{1}$, it follows that $h h^{-}=h^{-} h=\mathrm{id}_{M}$. Hence $h$ is a homeomorphism, so the theorem follows with $U=U_{2} \cap H$.

\section{REFERENCE}

1. R. Abraham, Lectures of Smale on differential topology, Lecture notes, Columbia University, New York, 1962.

\section{PART II. FLOWS}

II.1. Introduction to flows. We shift now our survey to the case of the group $G=R$, the real numbers, acting on a manifold $M$, which for simplicity we will assume compact most of the time. Thus we are studying a 1-parameter group of diffeomorphisms, $\phi: R \rightarrow \operatorname{Diff}(M)$ with $\phi_{0}: M \rightarrow M$ the identity and $\phi_{t} \phi_{s}=\phi_{t+s}$. We will call this set of data, or $\phi$, simply a flow. A flow $\phi_{t}: M \rightarrow M$ defines (or generates) a tangent vector field on $M$; i.e., for each $x \in M$ define $X(x) \in T_{x}(M)$ by

$$
\left.d \phi_{t}(x) / d t\right]_{t=0}=X(x) .
$$

Thus $X(x)$ is a tangent vector at $x$ on $M$ and $\phi_{t}(x)$ is the solution of the ordinary differential equation (1.1) with initial condition $\phi_{0}(x)=x$. Then the orbit (of $\phi$ ) through $x, t \rightarrow \phi_{t}(x)$ coincides with the solution of the first order, autonomous (i.e., $X(x)$ doesn't depend on $t$ ), ordinary differential equation (1.1).

Conversely, given an ordinary differential equation, simple methods reduce it to the first order autonomous case and thus one obtains the situation in (1.1) with $X(x)$ given. The fundamental existence theorems of ordinary differential equations (see [25], [56]) yield a solution $\phi_{t}(x)$ such that $\phi_{0}(x)=x$, at least locally, i.e., for $|t|<\epsilon$. Furthermore these local solutions may be pieced together (see [56]) and frequently this leads to a flow on $M$. Certainly if $M$ is compact, every (smooth of course) tangent vector field defines a unique flow in this way. In the noncompact case one may change the vector field by a scalar factor to obtain one which defines a global flow. We will consider here only the case where $\phi_{t}: M \rightarrow M$ is defined for all $t$, or an action of $R$ on $M$, i.e., a flow.

Most of Part II is the carrying over of Part I to this slightly more complicated case. We will emphasize some of the special features and new interesting problems encountered in this 1-parameter case.

There are three possible types of orbits of a flow $\phi_{t}: M \rightarrow M$. First 
$x$ is fixed point of the flow if $\phi_{t}(x)=x$ all $t \in R$. A fixed point $x$ can also be characterized as a zero of the vector field defined by $\phi$. Secondly a closed orbit of $\phi_{t}: M \rightarrow M$ is the orbit through some $x$ with $\phi_{t}(x)=x$ some $t \neq 0$. Usually a closed orbit is taken to mean exclusion of the fixed point case so there is a minimum period $t_{0}>0$ such that $\phi_{t_{0}}(x)=x$. Finally if $t \rightarrow \phi_{t}(x)$ is injective, then the orbit through $x$ is not one of the above types and could be called an ordinary orbit.

In topologizing actions of $R$ we assume $M$ is compact. Then the flows as we saw above correspond precisely to tangent vector fields on $M$. The $C^{r}, r>0$, vector fields on $M$ form a linear space and with a $C^{r}$ norm, $r<\infty$, a Banach space which we denote by $\chi(M)$. A generic property of flows will be a property true for a Baire set in $\chi(M)$. The most obvious generic property is that the set of zeros of $X \in \chi(M)$ is finite [114].

Proceeding as in $\$ I .1$ we look for a suitable equivalence between two flows $\phi_{t}$ and $\psi_{t}$ on $M$. A conjugacy between $\phi_{t}$ and $\psi_{t}$ is a homeomorphism $h: M \rightarrow M$ such that $h \phi_{t}(x)=\psi_{t}(h x)$. Such an equivalence relation preserves the minimum period of a periodic orbit and thus a conjugacy class will not in general be invariant under perturbation. This implies the need of a weaker notion of equivalence. We say that flows $\phi_{t}, \psi_{t}$ are topologically equivalent if there is a homeomorphism of $M$ sending orbits of $\phi_{t}$ into orbits of $\psi_{t}$. If perturbations in $\chi(M)$ do not change the topological equivalence class of $X \in \chi(M)$, then $X$ is called structurally stable. This concept was introduced in 1937 [6] by Andronov and Pontrjagin for ordinary differential equations on the 2-dimensional disk. On compact 2-dimensional manifolds, the structurally stable flows form a dense open set, simply characterized (Peixoto [84], see also \$II.2). However in every dimension higher than three there exist compact manifolds on which the structurally stable flows are not dense [116] (see also [87]). Parallel to Part I this leads to a weakening of topological equivalence as follows.

For a flow $\phi_{t}$ on $M, \mathrm{G}$. D. Birkhoff [15] has defined $x \in M$ to be a wandering point if there is some neighborhood $U$ of $x$ in $M$ with $\left(U_{|t|>t_{0}} \phi_{t}(U)\right) \cap U=\varnothing$ for some $t_{0}>0$.

The nonwandering points (those which are not wandering) form a closed invariant subset of $M$ denoted by $\Omega=\Omega\left(\phi_{t}\right)$. We will say that flows $\phi_{t}, \psi_{t}$ are topologically equivalent on $\Omega$ if there is an orbit preserving homeomorphism $h: \Omega\left(\phi_{t}\right) \rightarrow \Omega\left(\psi_{t}\right)$. Then $\phi_{t}$ is $\Omega$-stable if sufficiently small perturbations (measured in terms of the corresponding $X \in \chi(M)$ of course) are topologically equivalent on $\Omega$ to $\phi_{t}$. It is an important problem to discover whether $\Omega$-stable flows are dense in $\chi(M)$. 
We end this section by giving a direct relation between the flows discussed here and the diffeomorphism questions of Part I, [114].

A compact submanifold $\Sigma$ of codimension one of a compact manifold $M$ is called a cross-section for a flow $\phi_{t}$ on $M$ if $\Sigma$ intersects every orbit, has transversal intersection with the flow and whenever $x \in \Sigma$, $\phi_{t}(x) \in \Sigma$ for some $t>0$. Then $\phi_{t}$ induces a diffeomorphism $f: \Sigma \rightarrow \Sigma$ by $f(x)=\phi_{t_{0}}(x)$ where $t_{0}$ is the first $t>0$ with $\phi_{t}(x) \in \Sigma$ (see [114] for more details). The topological equivalence class of $\phi_{t}$ is determined by the topological conjugacy class of $f$. Orbits of $f$ are in a natural 1-1 correspondence with those of $\phi_{t}$ by $\left\{f^{m}(x) \mid m \in Z\right\} \rightarrow\left\{\phi_{t}(x) \mid t \in R\right\}$, each $x \in \Sigma$. Compact orbits are preserved under this correspondence; thus periodic points of $f$ correspond to closed orbits of $\phi_{t}$. There can be no fixed points of $\phi_{t}$ when there is a cross-section. Cross-sections were used by Poincaré and Birkhoff (see, e.g., [19]).

There is a converse construction of some importance. Given a diffeomorphism $f$ of a (compact) manifold $\Sigma$ we will construct a flow, canonically, on a manifold $M_{0}$ of one dimension higher, called the suspension of $f$. This goes as follows. Let $\alpha: \Sigma \times R \rightarrow \Sigma \times R$ be defined by $\alpha(x, u)=(f(x), u+1)$. Then $\left\{\alpha^{m}\right\}=Z$ operates freely on $\Sigma \times R$ and the orbit space is a manifold $M_{0}$. Furthermore the flow $\phi_{t}: \Sigma \times R$ $\rightarrow \Sigma \times R$ defined by $\psi_{t}(x, u)=(x, u+t)$ induces a flow $\phi_{t}$ on $M_{0}$ which is our suspension of $f$. Clearly $M_{0}$ will have a cross-section $\Sigma_{0}$ $=\pi(\Sigma \times 0) \subset M$ where $\pi: \Sigma \times R \rightarrow M_{0}$ is the quotient map. It is easy to check that the associated diffeomorphism of $\left(\phi_{t}, \Sigma_{0}\right), f_{0}: \Sigma_{0} \rightarrow \Sigma_{0}$ is differentiably conjugate to our initial $f: \Sigma \rightarrow \Sigma$. Furthermore if an arbitrary flow $\phi_{t}: M \rightarrow \Sigma$ has a cross-section $f: \Sigma \rightarrow \Sigma$ whose suspension is $\phi_{t}^{\prime}: M_{0} \rightarrow M_{0}$, then $\phi_{t}$ and $\phi_{t}^{\prime}$ are equivalent by an orbit preserving homeomorphism.

This notion of suspension is useful because it allows one immediately to transfer all the examples in Part I, i.e., the diffeomorphisms of $\S I .2$, Anosov diffeomorphisms as well as those of $\$$ I.5 and I.6, to examples of flows. From the above remarks, all the stability properties of the diffeomorphism examples are kept by the suspended flows.

II.2. The simplest examples of $\Omega$-stable flows. We will say that a fixed point $x$ of the flow $\phi_{t}: M \rightarrow M$ is hyperbolic if $x$ is a hyperbolic fixed point of the diffeomorphism $\phi_{1}: M \rightarrow M$. An alternate way of saying this is as follows: If $x$ is a fixed point of the flow $\phi_{t}: M \rightarrow M$, then the derivative $D \phi_{t}(x): T_{x}(M) \rightarrow T_{x}(M)$ defines a linear representation of the real line and so can be written in the form $D \phi_{t}(x)$ $=e^{t A}$ where $A$ is a linear endomorphism of $T_{x}(M)$. Then $x$ is hyper- 
bolic if and only if none of the eigenvalues of $A$ have real part equal to zero.

(2.1) Propositjon. If $x$ is a hyperbolic fixed point for the flow $\phi_{t}$, the stable manifold $W^{s}(x)$ of $x$ relative to $\phi_{1}$ is invariant under $\phi_{t}$ for every $t$ and is contracting for every $t>0$.

Then $W^{s}(x)$ will be called the stable manifold of $x$ for the flow $\phi_{t}$. One may apply I. (2.1) to obtain properties of $W^{s}(x)$.

Now suppose that $x \in M$ is in a closed orbit $\gamma$ of the flow $\phi_{t}: M \rightarrow M$. There is a submanifold $V$ of codimension one passing through $x$ and transversal to $\gamma$. Then $V$ serves as a local version of the cross-section of §II.1, defining a local diffeomorphism $f: U \rightarrow V, f(x)=x$, where $U$ is a neighborhood of $x$ in $V$. We say that $\gamma$ is a hyperbolic closed orbit of $\phi_{t}$ whenever $x$ is a hyperbolic fixed point of $f$. It is easily checked that this definition is independent of the choices $x \in \gamma$ and $V$ (see [114]). The local stable manifold $W_{\text {loo }}^{s}(x, f)$ of $x$ for $f$ in $U$ defines the stable manifold $W^{s}(\gamma)$ of $\gamma$ by $W^{s}(\gamma)=\bigcup_{t \in R} \phi_{t}\left(W_{\mathrm{loc}}^{s}(x, f)\right)$. Then $W^{s}(\gamma)$ is a 1-1 immersed cell bundle over $S^{\prime}$ (either a cylinder $R^{k} \times S^{1}$, or a generalized Möbius band). For more details see [114].

The unstable manifolds of hyperbolic fixed points and closed orbits of $\phi_{t}$ are defined as the stable manifolds of $\psi_{t}=\phi_{-t}$.

For the suspension of a toral diffeomorphism ( $\$ 1.3)$, the closed orbits are hyperbolic and dense in $M$; but hyperbolic fixed points of any flow are necessarily isolated fixed points.

We now describe the analogue of the diffeomorphisms of $\$ 1.2$ as flows $\phi_{t}: M \rightarrow M, M$ compact, which satisfy

(2.2) (1) $\Omega\left(\phi_{t}\right)$ is the union of a finite number of fixed points $x_{1}, \cdots, x_{m}$ and a finite number of closed orbits $\gamma_{1}$, $\cdots, \gamma_{n}$ of $\phi_{t}$.

(2) The $x_{i}, \gamma_{j}$ are all hyperbolic.

(3) The stable manifolds and unstable manifolds of the $x_{i}$, $\beta_{j}$ intersect each other only transversally.

(2.3) Theorem [109]. Suppose the flow $\phi_{t}: M \rightarrow M$ satisfies (2.2). Then (a) Each stable manifold $W_{k}^{s}$ of the $x_{i}$ and $\gamma_{j}$ is imbedded and $M=\mathrm{U}_{k=1}^{m+n} W_{k}^{s}$ (disjoint union).

(b) The closure of one $W_{k}^{s}$ is the union of certain $W_{l}^{s}$. Let $W_{l}^{s} \leqq W_{k}^{s}$ if $W_{l}^{s}$ is in the closure of $W_{k}^{s}$. Then $\leqq$ is a partial ordering. If $W_{l}^{s} \leqq W_{k}^{s}$ then $\operatorname{dim} W_{l}^{s} \leqq \operatorname{dim} W_{k}^{s}$.

(c) One has the following Morse inequalities: 


$$
\begin{gathered}
M_{0} \geqq B_{0}, \\
M_{1}-M_{0} \geqq B_{1}-B_{0}, \\
\cdot \cdot \cdot \cdot \cdot \cdot \cdot \cdot \cdot \cdot \cdot \\
\sum_{k=0}^{n}(-1)^{k} M_{k}=\sum_{k=0}^{n}(-1)^{k} B_{k} .
\end{gathered}
$$

Here $B_{i}$ is the $i$ th betti number coefficients $Z_{2}$, and $M_{i}=a_{i}+b_{i}$ $+b_{i+1}$ where $a_{i}$ is the number of $x_{j}$ with dimension $W^{s}\left(x_{j}\right)=i$ and $b_{i}$ is the number of $\gamma_{j}$ with dimension $W^{s}\left(\gamma_{j}\right)=i+1$.

One can see that II.(2.3) is quite analogous to I.(2.3). There are a couple of special features in the present situation however. For example

(2.4) Theorem (Peixoto [84]). If $\operatorname{dim} M=2$, then the flow $\phi_{t}$ satisfies (2.2) if and only if it is structurally stable.

In this case the corresponding $X \in \chi(M)$ form an open and dense set.

This theorem gives a rough but quite good picture of flows on compact 2-manifolds. It solves the first basic problem for 2-dimensional flows.

A gradient flow $\phi_{t}: M \rightarrow M$ on a compact Riemannian manifold is defined by a $C^{r}$ function $f: M \rightarrow R$ in the following way. The derivative $D f(x)$ of $f$ at $x$ is a cotangent vector at $x$ and the Riemannian metric converts this into a tangent vector $X(x)=(\operatorname{grad} f)(x)$ at $x$. By the familiar procedure (§II.1) from $X(x)$ we obtain our gradient flow $\phi_{t}$.

(2.5) TheOREM [109]. The flows on any given $M$ satisfying (2.2) contain an open and dense subset of all gradient flows.

Since every manifold possesses Riemannian metrics, we see that from (2.5) every manifold exhibits flows satisfying (2.2). Recall the existence of the diffeomorphisms of $\$ I .2$ was obtained in this way. Theorem (2.5) gives the bridge between the usual Morse theory for functions on manifolds and the work in this section. This even brings the subject here close to handlebody theory in differential topology and Poincaré duality on a manifold (this is the duality between the stable and unstable manifolds of a gradient flow).

See [95] for one definitely nongradient type example satisfying (2.2). See also [63], [97] for related papers.

$I$ have just received a manuscript of $K$. Meyer [15] in which 
"energy functions" are constructed for the flows described in this section.

II.3. Anosov flows. Consider first 1-parameter groups of vector space bundle automorphisms $\phi_{t}: E \rightarrow E$. Here $E$ is a vector space bundle and $\phi_{t}$ is a flow on $E$ such that for each $t, \phi_{t}: E \rightarrow E$ is a bundle automorphism (i.e., linear on fibers). For example if $\psi_{t}: M \rightarrow M$ is a flow on a manifold, the derivatives at each $t, \phi_{t}=D \psi_{t}: T(M) \rightarrow T(M)$, define a 1-parameter group of vector space bundle automorphisms. Assuming $E$ is a Riemannian vector space bundle, say that such a flow $\phi_{t}: E \rightarrow E$ is contracting if there are constants $c, \lambda>0$ such that $\left\|\phi_{t}(v)\right\| \leqq c e^{-\lambda t}$, all $v \in E, t>0$.

Then $\phi_{t}$ is expanding if $\phi_{-t}$ is contracting and this is equivalent to the existence of $c_{1}>0, \mu>0$ such that $\left\|\phi_{t}(v)\right\| \geqq c_{1} e^{\mu t}$ all $t>0, v \in E$ (compare $\S 1.3$ ).

An Anosov flow on a complete Riemannian manifold $M$ (or just a manifold in case $M$ is compact) is a flow $\phi_{t}$ whose induced flow $D \phi_{t}: T(M) \rightarrow T(M)$ on the tangent bundle is hyperbolic in the following sense: The tangent bundle $T(M)$ can be written as the Whitney sum of 3 invariant subbundles, $T(M)=E_{1}+E_{2}+E_{3}$ where on $E^{u}=E_{1}$, $\phi_{t}$ is expanding, on $E^{s}=E_{2}, \phi_{t}$ is contracting and $E_{3}$ is the 1-dimensional bundle defined by differentiating $\phi_{t}$ with respect to $t$.

Examples of Anosov flows are obtained readily from $\$ 1.3$ and the following easily proved proposition.

(3.1) Proposition. If $f: M \rightarrow M$ is an Anosov diffeomorphism of a compact manifold, then the suspension of $f$ is an Anosov flow.

Another important class of examples of Anosov flows are the geodesic flows on the tangent bundles of Riemannian manifolds of negative (possibly varying) curvature (see [8], [13]).

(3.2) Theorem (Anosov [9]). If $\phi_{t}: M \rightarrow M$ is an Anosov flow of a compact manifold it is structurally stable. Also if $\Omega=M$, the periodic orbits will be dense. Finally if there is an (Lebesgue) invariant measure, then $\phi_{t}$ is ergodic.

Applied to the geodesic flows on the tangent bundles of manifolds $M$ with negative curvature, (3.2) yields ergodicity, thus solving an old problem. The constant negative curvature case as well as the case of two dimensional $M$ had been done earlier by G. Hedlund [42] and E. Hopf [45], [46]. See also [34] and [64].

Again as in $\S I .3$. there is the very important problem of finding all Anosov flows on compact $M$ (especially when $\Omega=M$ ). Progress on this problem might contribute to the problem of what manifolds can 
have Riemannian metrics of negative curvature. Is this class bigger than the class of manifolds which possess Riemannian metrics of constant negative curvature? On this point see the problem of Calabi in $[51]$.

II.4. On counting closed orbits. For counting the fixed points (at least algebraically) of a diffeomorphism, the Lefschetz Trace Formula provides a satisfactory method (see §I.4). This also applies to periodic points, and for suspended flows, these methods will give us some answers as to the nature of closed orbits. For flows in general, it is an outstanding problem to find methods which will tell if there are closed orbits and how many.

Seifert's problem [105] is the best known question exemplifying this lack of knowledge. That is, does a flow on $S^{3}$ (continuous or differentiable) have a closed orbit or a fixed point? A related question is: does $X$, a smooth vector field on $D^{2} \times S^{1}$, the 2-disk cross the circle, transversal to the boundary, have a closed orbit or a singular point? Related to these questions are papers of Fuller [32], and A. Schwartz [104].

Thus an analogue of the zeta function for diffeomorphisms of \$I.4 seems quite remote for flows. However we will mention a wild idea in this direction.

Let $\Gamma=\Gamma\left(\phi_{t}\right)$ be the set of closed orbits of the flow $\phi_{t}: M \rightarrow M$ where we will assume $M$ to be compact and that there are no fixed points. For $\gamma \in \Gamma$, define $l(\gamma)$ to be the period (minimal period, that is) of $\gamma$, i.e., $l(\gamma)$ is the first $t>0$ such that $\phi_{t}(x)=x$ for some $x \in \gamma$. We will assume then that the flow satisfies the generic property, $\{\gamma \in \Gamma \mid l(\gamma) \leqq c\}$ is finite for each positive $c$ (that this is generic follows from II.(5.6)).

Then define formally (another zeta function!) $Z(s)$ to be the infinite product

$$
Z(s)=\prod_{\gamma \in \Gamma} \prod_{k=0}^{\infty}\left(1-[\exp l(\gamma)]^{-8-k}\right) .
$$

The question is: does $Z(s)$ have nice properties for any general class of flows $\phi$ ? In this direction we consider the case that $\phi$ is the suspension of a diffeomorphism $f: V \rightarrow V$ where the zeta function (of Weil, Artin-Mazur, \$I.4) is rational.

(4.1) THEOREM. If the zeta function of $f: V \rightarrow V$ is rational, then $Z_{\phi}(s)=Z(s)$ where $\phi$ is the suspension of $f$ converges in a half-plane to an analytic function of $s$, and has an analytic continuation to a meromorphic function. Furthermore the zeros and poles of this meromorphic function can be computed explicitly in terms of those of $\zeta_{f}$. 
Proof (Largely Due to Narasimhan). If $K_{m}=K_{m}(f)$, $m=1,2,3, \ldots$ (as in $\S I .4$ ) denotes the number of periodic points of $f$ of minimum period $m$, we get directly from the definition of $Z(s)$

$$
Z(s)=\prod_{m=1}^{\infty} \prod_{k=1}^{\infty}\left(1-e^{-m(s+k)}\right)^{K_{m} / m}
$$

Let

$$
W(s)=\prod_{m=1}^{\infty}\left(1-e^{-m s}\right)^{K_{m} / m}
$$

Then

$$
\begin{aligned}
-\log W(s) & =\sum_{m=1}^{\infty} \frac{1}{m} K_{m} \log \left(\frac{1}{1-e^{-m s}}\right) \\
& =\sum_{m, r \geq 1} \frac{1}{m r} K_{m} e^{-m r s} \\
& =\sum_{n \geqq 1} \frac{1}{n} e^{-n s} \sum_{m / n} K_{m} .
\end{aligned}
$$

Assuming at first that the zeta function $\zeta(t)$ of $f$ is of the form $\zeta(t)=(1-\lambda t)^{-1}$, we have (I. (4.1), (4.8)) $\quad \sum_{m / n} K_{m}=\lambda^{n}$. Thus $-\log W(s)=\sum(1 / n)\left(\lambda / e^{s}\right)^{n}=-\log \left(1-\lambda / e^{s}\right)$ or $W(s)=1-\lambda / e^{s}$ and

$$
Z(s)=\prod_{k=0}^{\infty} W(s+k)=\prod_{k=0}^{\infty}\left(1-\lambda / e^{s+k}\right) .
$$

Then we can see that $Z(s)$ is entire because it is the uniform limit, in every compact set, of entire functions. Incidentally one sees from the explicit form of $Z(s)$, a functional equation $Z(s+1)=Z(s) e^{s} /\left(e^{s}-\lambda\right)$. Finally the zeros are clearly the solutions of $e^{s+\mu}=\lambda, k=0,1,2, \cdots$ or $s+k=\log \lambda+2 \pi i n, n \in Z$.

In the general case we have $\zeta(t)=\prod_{i, j}\left(1-\mu_{j} t\right) /\left(1-\lambda_{i} t\right)$ and $\sum_{m / n} K_{m}=\sum_{i} \lambda_{i}^{n}-\sum_{j} \mu_{j}^{n}$. Thus we obtain - log $W(s)$ $=-\log \prod_{i, j}\left(1-\lambda_{i} / e^{s}\right)\left(1-\mu_{j} / e^{s}\right)^{-1}$, so $Z(s)=\prod_{i, j} \prod_{k=0}^{\infty}\left(1-\lambda_{i} / e^{s+k}\right)$ $\cdot\left(1-\mu_{j} / e^{s+k}\right)^{-1}$. The zeros are of the form $s=\log \lambda_{i}+2 \pi n i-k$ and the poles $s=\log \mu_{j}+2 \pi n i-k$ (distinguish the $i$ 's!). This proves (4.1).

The following question then arises. Suppose $\phi_{t}: M \rightarrow M$ is the suspension of $f$ as in (4.1) with $\zeta_{f}$ rational and $f$ satisfying Axioms $\mathrm{A}$ and $\mathrm{B}$ of $\S 1.6$. Suppose even that $M$ is the 2-dimensional toral diffeomorphism. Now let $\psi_{t}: M \rightarrow M$ be close to $\phi_{t}$. Does $Z_{\psi_{t}}(s)$ have a meromorphic continuation to all of $\boldsymbol{C}$ ? An affirmative answer would be roughly necessary and sufficient condition for $Z(s)$ to be useful. I must 
admit a positive answer would be a little shocking! A way of looking at this problem is the following. The canonical cross-section $\Sigma$ for $\phi_{t}$ is also a cross-section for $\psi_{t}$ and the time of first return for $\psi_{t}$ is defined by a smooth function $\rho: \Sigma \rightarrow R^{+}$( $R^{+}$the positive reals) which will be close to the constant function 1 . There is a natural 1-1 correspondence $\gamma \rightarrow \gamma^{\prime}, \Gamma \rightarrow \Gamma^{\prime}$ from the set of closed orbits of $\phi_{t}$ to those of $\psi_{t}$ using $\Omega$-stability.

Let $\lambda_{\gamma}=l\left(\gamma^{\prime}\right)$, so $\lambda_{\gamma}=\sum_{x_{i} \in \gamma^{\prime} \cap \Sigma} \rho\left(x_{i}\right)$ and $-\log W_{1}(s)$ $=\sum_{\gamma, r}(1 / r) e^{-\lambda \gamma r s}$ where $W_{1}(s)$ corresponds to the $W(s)$ of the previous proof. Is there sufficient regularity in the $\lambda_{\gamma}$ to continue $W_{1}$ meromorphically?

There are two other remarks we wish to make about $Z(s)$. First if $\phi_{t}$ is the geodesic flow for a 2-manifold of constant negative curvature, then $Z(s)$ is meromorphic. In this case it is precisely the Selberg zeta function [106], which Selberg defined directly in terms of $\operatorname{SL}(2, R)$ and a certain uniform discrete subgroup $\Gamma$. Selberg proved that it is meromorphic in this case and found its zeros and poles as well. Sinai and Langlands pointed out to me this interpretation of the Selberg zeta function and this motivated my using it here.

Finally we pose the question, how generally do flows have the $l(\gamma)$ growing slowly enough so that $Z(s)$ has a half plane of convergence?

II.5. Spectral decomposition of flows. One can extend Axioms A and B of $\$ I .6$ to flows. This goes as follows. For flows $\phi_{t}$ on compact manifolds $M$, we have

(5.1) Ахгом A'. The fixed points of $\phi_{t}$ are each hyperbolic. The nonwandering points $\Omega$ consist of this finite set of fixed points $F$ and the closure $\Lambda$ of the closed orbits; $\Lambda$ and $F$ are disjoint. Finally the derived flow restricted to the tangent bundle restricted to $\Lambda, D \phi_{t}: T_{\Lambda}(M) \rightarrow T_{\Lambda}(M)$ is hyperbolic (defined analogously to the Anosov flow in \$II.3).

Topologically transitive for a flow again means that there is a dense orbit.

(5.2) Theorem (Spectral Decomposition). If $\phi_{t}: M \rightarrow M$ satisfies Axiom $\mathrm{A}^{\prime}$, then $\Omega$ can be written uniquely as the disjoint union $\Omega_{1} \cup \Omega_{2}$ $\cup \ldots \cup \Omega_{k}$ where each $\Omega_{i}$ is closed, invariant and each $\phi_{t}: \Omega_{i} \rightarrow \Omega_{i}$ is topologically transitive.

(5.3) Corollary. $M=\bigcup_{i=1}^{k} W^{s}\left(\Omega_{i}\right)$ (disjoint union, canonically) where each $W^{s}\left(\Omega_{i}\right)=\left\{x \in M \mid \phi_{t}(x) \rightarrow \Omega_{i}\right\}$.

(5.4) Axrom $\mathrm{B}^{\prime}$. Conditions and notations as above, if $W^{s}\left(\Omega_{i}\right)$ $\cap W^{k}\left(\Omega_{j}\right) \neq \varnothing$, then there exist periodic orbits (or fixed points) $\gamma$ in 
$\Omega_{i}, \sigma$ in $\Omega_{j}$ such that $W^{s}(\gamma)$ and $W^{k}(\sigma)$ have a point of transversal intersection.

The following seems to be a theorem although I haven't written out the details.

(5.5) If $\phi_{t}: M \rightarrow M$ satisfies Axioms $\mathrm{A}^{\prime}$ and $\mathrm{B}^{\prime}$ then $\phi_{t}$ is $\Omega$-stable. One also obtains the openness, filtration, and partial ordering as in §I.6.

The approximation theorems are quite parallel to those referred to in $\$ 1.6$ with the same references in fact.

(5.6) THEOREM [55] AND [114]. The property of flows that the fixed points $x_{i}$ and closed orbits $\gamma_{j}$ are all hyperbolic is generic. Furthermore generically, the stable and unstable manifolds of the $x_{i}, \gamma_{j}$ intersect each other only transversally.

(5.7) Theorem (Pugh [91]). In the Banach space of $C^{\prime}$ vector fields (or flows), there is a Baire set with the property that the fixed points and closed orbits are dense in $\Omega$.

If $\phi_{t}: M \rightarrow M, \psi_{t}: V \rightarrow V$ there is defined naturally the product flow $\phi_{t} \times \psi_{t}: M \times V \rightarrow M \times V$. Note that the product of two (or more) flows containing closed orbits of positive period will contain an invariant torus which will make this product not $\Omega$-stable. For gradient flows (nondegenerate) the situation is different and simpler; the product is in this case $\Omega$-stable.

Note that one obtains the example showing that structurally stable flows are not dense, by simply suspending the example for diffeomorphisms.

All the material in $\$ I .5$ about homoclinic points and symbolic flows can be suspended to obtain similar results on flows. As mentioned there, I first ran into this phenomena in that form, i.e., in trying to understand Van der Pol's equation (with forcing term). See also [107] for these questions discussed in the flow framework.

\section{PART III. MORE ON FLOWS}

III.1. Flows with conditions imposed. In this section, we discuss some of the problems encountered in attempting to carry over Parts I and II to flows which satisfy certain constraints, e.g., of the type occurring in classical mechanics. Essentially nothing has been done in this direction, so we just mention some background material, related recent results, and some problems.

The main class of flows, beyond the unrestricted ones we have been 
discussing up to this point, are the Hamiltonian flows. Abstractly speaking, a Hamiltonian flow is defined on a symplectic manifold, and this proceeds as follows.

A symplectic structure on a manifold $M$ is a 2 -form $\theta$ defined on $M$ such that $d \theta=0$, and at each point of $M, \theta$ is nondegenerate; nondegeneracy of $\theta$ at $x \in M$ means that the map $Q: T_{x}(M) \rightarrow T_{x}^{*}(M)$ is an isomorphism from the tangent space at $x$ to its dual where $Q(X)(Y)$ $=\theta(X, Y), X, Y \in T_{x}(M)$ (for a complete discussion of this material, see $[1],[123])$. We then say that $M$ is a symplectic manifold. It follows that $\operatorname{dim} M$ is even. Thus on a symplectic manifold, there is a 1-1 correspondence between 1 -forms and vector fields.

Now if $H: M \rightarrow R$ is a differentiable function (a "Hamiltonian" function), its derivative $D H(x) \in T_{x}^{*}(M)$ defines a 1 -form, which via $Q$ we may consider as a vector field, say $X_{H}$. The flow $\phi_{t}$ generated by $X_{H}$ (at least locally) is called the Hamiltonian flow defined by $H$. It can be checked that $\phi_{t}$ leaves $\theta$ invariant. In fact, by reasons converse to the above, it is important to consider directly those flows (which we will again call Hamiltonian) $\phi_{t}$, say, defined for all $t$, on a symplectic manifold preserving the symplectic form. Then the natural global problem for Hamiltonian flows becomes

(1.1) Problem. Given a symplectic manifold $M$, find a Baire set $\Theta$ of all flows which preserve the symplectic form, so that if $\phi_{t}$ is in $B$, one can describe the global orbit structure of $\phi_{t}$.

If $M$ is compact, one may conveniently consider the Hamiltonian flows $\mathcal{H}$ as a subspace of all vector fields, $\chi(M)$ with the $C^{r}$ topology.

Note that a Hamiltonian flow, $\phi_{t}$, leaves a volume on $M$ invariant, namely the form obtained by wedging the symplectic form $\theta$ with itself $n=\frac{1}{2} \operatorname{dim} M$ times. Thus it follows that in case $M$ is compact, that the set of nonwandering points, $\Omega$ is equal to all of $M$.

One has a similar problem, also directly motivated by classical mechanics, for a single diffeomorphism.

(1.2) Problem. What is the orbit structure of some Baire set of diffeomorphisms $f$ of a compact symplectic manifold which preserve the symplectic 2 -form?

Of course in studying these problems, one is only permitted perturbations of $f$ to $f^{\prime}$ which also keep $\theta$ invariant. The first (and still unsolved) problem that one encounters here is to understand a local problem, the orbit structure in the neighborhood of a fixed point $x$ of $f$. The difficulty is that the symplectic condition on $f$ means that for the derivative $D(x)$, hyperbolicity is not a generic property. For example, if $\operatorname{dim} M=2, f$ preserves a volume and $D f(x): T_{x}(M)$ 
$\rightarrow T_{x}(M)$ has determinant 1 . One may classify these linear transformations into the hyperbolic and elliptic types. The hyperbolic is the one already discussed with eigenvalues $\lambda>1$ and $1 / \lambda$. The elliptic case is a nontrivial rotation of the plane. In the elliptic case in general, there exist no coordinates in the neighborhood of $x$ in which $f$ becomes linear, and only recently in this local 2-dimensional problem has one even begun to understand what is going on.

Birkhoff, e.g. [18], had believed that volume preserving transformations of compact 2-manifolds were ergodic (as well as Hamiltonian transformations more generally) "in the general case" and based much of his work on this hypothesis. (Recall ergodic means there are no invariant sets of positive measure with measure less than that of $M$.) Through the work of Kolmogoroff, Arnold, Moser, [52], [10], [71], we know now that this is not the case. If $x$ is an elliptic fixed point of $C^{\infty} f: M^{2} \rightarrow M^{2}$, then generically, there is an invariant circle in every neighborhood of $x$ and thus $f$ cannot be ergodic [70].

In the $2 n$-dimensional analogous problem there is an invariant $n$-dimensional torus in any neighborhood of $x$ and the diffeomorphism is not ergodic. However one still has not yet a topological description in the neighborhood of an elliptic fixed point of a Hamiltonian diffeomorphism and thus it seems especially difficult to know how to proceed as in the first parts of the survey. Furthermore, the recent work of Arnold and Moser on the Hamiltonian case is still fairly local; the global Hamiltonian picture seems remote. We remark, though, that the examples of geodesic flows on manifolds of negative curvature are Hamiltonian and in this case, ( $\$ I I .3)$, the flow is ergodic and structurally stable (on each level surface of the Hamiltonian).

We make three last comments on the Hamiltonian problem. First an elliptic point of a Hamiltonian diffeomorphism, say in 2 dimensions, where the derivative is a rational rotation, is degenerate. This is one reason why one must work with Baire sets of Hamiltonian diffeomorphisms, not open dense sets. Similarly one cannot expect these diffeomorphisms to be $\Omega$-stable, as in Part I. Secondly, we remark that Pugh has shown that his closing lemma applies to prove the periodic orbits are dense in the compact Hamiltonian case [93]. Lastly it should be said that in practice, or in engineering, the differential equations, because of friction, are no longer Hamiltonian and could be closer to those described in Parts I and II. In this connection see [85].

After the Hamiltonian problems, the next most interesting case to consider might well be volume preserving diffeomorphisms. These coincide in dimension two with the Hamiltonian ones. 
Volume preserving diffeomorphisms have not been studied from our point of view (although, see [122]). For $\operatorname{dim} M>2$, however, none of the Hamiltonian objections apply and in fact the hyperbolic linear volume preserving maps are dense and open among all volume preserving linear maps; very possibly in the higher dimensional case, volume preserving diffeomorphisms might be amenable to study by the methods of Part I. A first question could be to prove I.(6.7) for volume preserving diffeomorphisms.

For every volume preserving diffeomorphism $f$ of a compact manifold, $\Omega(f)=M$. Presumably, Pugh's method would show the periodic points are dense. Is $f$ ergodic, a generic property in this context? Oxtoby and Ulam [78] prove such an ergodicity theorem for homeomorphisms.

One can also ask whether the program of Parts I and II could be carried out for ordinary differential equations of higher order, say second order to begin with; see [56], [123] for a coordinate free definition of 2 nd order differential equations. This hasn't been investigated as far as I know. The same applies to diffeomorphisms or flows of infinite dimensional manifolds.

Holomorphic diffeomorphisms of a complex manifold are much more rigid, but I think that the orbit structure is not generally understood. G. Julia's prize memoir [50] is related to this subject. It concerns holomorphic endomorphisms of the Riemann sphere.

III.2. Some other work on flows. Here we mainly remark on a couple of recent results on flows which are not so directly related to the preceding.

The question of existence for minimal sets poses interesting problems to the global analyst. A compact manifold (or even space) $M$ is a minimal set for the flow $\phi_{t}: M \rightarrow M$ if there is no proper nonempty closed invariant subspace of $M$. Gottschalk [35] has given a survey of this subject. A main problem is: what $M$ can be the minimal set for some flow? It is not known if the 3 -sphere can be a minimal set.

A number of new examples of minimal flows are constructed from Lie groups in [12a]. See also [29] for examples on $S^{n} \times S^{1}$.

An important recent result is that of A. Schwartz [102] which generalizes both the Poincaré-Bendixson theorem for plane regions and Denjoy's theory of $C^{2}$ flows on the torus. The Schwartz theorem says that for any $C^{2}$ flow on a 2 -manifold, any (compact) minimal set is either a point, a closed orbit, or a 2-torus. Among other applications of Schwartz's methods, R. Sacksteder has shown that if $G$ is a finitely generated, finitely presented, discrete group $G$ acting $C^{2}$ 
freely on the circle, then the action is topologically conjugate to a group of rotations. See also [99]. Here acting freely means no $\phi_{0}: S^{1} \rightarrow S^{1}, g \in G$, has a fixed point.

There has been recently also interesting work on the subject of distal actions which we do not go into. Here $\phi_{t}: M \rightarrow M$, compact $M$, is distal, if in some metric, for any $x, y \in M, x \neq y$, there is an $\epsilon$ such that $d\left(\phi_{t}(x), \phi_{t}(y)\right)>\epsilon$ all $t \in G$. See for example [28], [33], [67].

\section{PART IV. OTHER LIE GROUPS}

IV.1. Action of an abelian Lie group. We consider briefly here the question of an abelian Lie group $G$ acting on a manifold when $G$ is more complicated than $Z$ or $R$.

Recall first that an action of a Lie group $G$ on a manifold is a homomorphism $\phi: G \rightarrow \operatorname{Diff}(M)$ such that the induced map $\Phi: G \times M$ $\rightarrow M$ defined by $\Phi(g, m)=\phi_{g}(m)$ is $C^{k}$. The orbit $O_{x}$ through $x \in M$ of such an action is the image of the map $p_{x}: G \rightarrow M$ defined by $p_{x}(g)$ $=\phi_{g}(x)$. The isotropy group $H_{x}$ of the action at $x$ is the set of elements $h \in G$ such that $\phi_{h}(x)=x$. Then $H_{x}$ is a closed subgroup of $G$ and $G / H_{x}$ is a homogeneous space of $G$. Induced from $p_{x}$ is a 1-1 immersion $q_{x}: G / H_{x} \rightarrow M$. Finally we remark that the $x$ orbit $O_{x}$ refers to $p_{x}$, $q_{x}, G / H_{x}$ or $q_{x}\left(G / H_{x}\right)$ at various times. If there is danger of confusion we will try to be more explicit.

A fixed point of the action is an orbit consisting of a single point. Actions $\phi_{g}, \psi_{g}$ are conjugate if there is a homeomorphism $h: M \rightarrow M$ such that $\phi_{g}(h x)=h\left(\psi_{g}(x)\right)$ for all $g \in G, x \in M$.

Returning to the abelian case, suppose $G$ is isomorphic to $Z+Z$. One may choose generators $f, g \in \operatorname{Diff}(M)$ of $G$, so $f g=g f$, and thus one is equivalently studying a pair of commuting diffeomorphisms. More generally one may study two commuting differentiable maps and actually the most studied of such problems perhaps has been the existence of a common fixed point for two commuting maps of the unit interval $I$ into itself. Very recently a counterexample has been found to this problem by $\mathrm{P}$. Huneke [49] and independently W. Boyce [22]. They each construct continuous maps $f, g: I \rightarrow I$, with $f g=g f$ and such that there is no $x \in I$ with $f(x)=x=g(x)$. These maps are not $C^{1}$ and thus the differentiable version of this problem remains open. In this direction, A. Schwartz [103] has the strongest result: If $f$ and $g$ are $C^{1}$ maps, $I \rightarrow I$, there is a fixed point of one which is periodic for the other.

Going back to the case of two commuting diffeomorphisms $g, f: M \rightarrow M$, observe $g$ is in the centralizer $Z(f)$ of $f$, i.e., $Z(f)$ $=\{g \in \operatorname{Diff}(M) \mid g f=f g\}$. Thus a first question in such a study could well be 
(1.1) Problem. What can be said about $Z(f)$ for $f \in \operatorname{Diff}(M)$ ? Under what conditions on $f$ is $\operatorname{dim} Z(f)<\infty$ ? Is $Z(f)=\left\{f^{m} \mid m \in Z\right\}$ a generic property?

Work of N. Kopell suggests that this last question may have an affirmative answer. Since a significant class of $\Omega$-stable diffeomorphisms ( $\S 1.2)$ are a finite union of contractions up to a finite power, it is important to know $Z(f)$ when $f$ is a contraction.

(1.2) Theorem (Kopell [53]). Suppose $C^{\infty} f: W \rightarrow W$ is a contraction. Thus at the unique fixed point $x$, derivative $D f(x): T_{x}(W) \rightarrow T_{x}(W)$ is a linear contraction. Then $Z(f)=\left\{g \in \operatorname{Diff}(W) \mid g f=f g, g \in C^{\infty}\right\}$ is a finite dimensional Lie group. If $f$ is linear, with a further nondegeneracy condition on the eigenvalues, then $g$ is linear. Finally for a dense open set of diffeomorphisms $f$ satisfying I.(2.2), $Z(f)=\left\{f^{m} \mid m \in Z\right\}$.

In the proof of the structural stability of an Anosov diffeomorphism (see the Appendix of Part I), one obtains at the same time that its centralizer is discrete, even in the group of homeomorphisms of $M$. Adler and Palais [5] have actually computed this centralizer for the toral diffeomorphisms. It would seem at least a reasonable conjecture that an open dense set of diffeomorphisms satisfying Axioms A and B (§I.6) have centralizer $Z(f)=\left\{f^{m} \mid m \in Z\right\}$.

Kopell [53] has studied commuting diffeomorphisms of the circle in more detail. Here, at least among those with periodic points, she has found a dense set of actions of $Z+Z$ for which the orbit structure can be understood. She also gives an example of commuting diffeomorphisms $f, g$ of $S^{\prime}$ with the following property: $g$ is the identity on an open set and for $C^{\prime}$ approximations $f^{\prime}, g^{\prime}$ of $f, g$ such that $f^{\prime} g^{\prime}=g^{\prime} f^{\prime}$, $g^{\prime}$ must also be the identity on some open set.

Further results on abelian actions are related to the question of degeneracy of some orbits when $R^{k}$ acts on a given manifold. By taking generators, an action of $R^{k}$ on $M$ corresponds to a set of $k$ tangent vector fields on $M$ which commute, or equivalently their bracket is zero. In this direction Lima [60] showed that if $R^{2}$ acts on a compact 2-manifold of nonzero Euler characteristic, there must be a fixed point or, equivalently, a common zero of the two generating vector fields. In a further paper [59] he showed that two commuting vector fields on $S^{3}$ are dependent at some point (see also Novikov-Arnold [76]). Extensions of this last theorem have been made to actions of $R^{k}$ on certain $M^{k+1}$ by Rosenberg, and Sacksteder [98], [100]. While on this subject it seems worthwhile to mention that closely related is the result of Novikov [77] who has shown that every foliation of dimension 2 on $S^{3}$ has a compact leaf. An account of the basic results in foliation theory is in Haefliger [41]. 
Recently Adler and McAndrew [4] have shown that the topological entropy of a Chebyshev polynomial is positive.

There has not been much work on actions of solvable or nilpotent Lie groups along the line of this section.

IV.2. The semisimple case. Here we make a few comments on the problem of studying the action of a semisimple group $G$ on a (compact) manifold. Discussions with $R$. Palais have been helpful here.

There is a vast literature on the subject of a Lie group $G$ acting on a manifold $M$ when $G$ is compact, acts transitively, or acts linearly. The reader can refer to [20], [66] for the case of compact $G$. We only remark that Palais [80] (see also [81]) has shown a strong form of structural stability for compact actions. Namely if an action $\psi_{g}$ is close to $\phi_{g}, \phi: G \rightarrow \operatorname{Diff}(M), G$ compact, these actions are conjugate by a diffeomorphism $h \in \operatorname{Diff}(M)$. Thus $\phi_{\theta}(h x)=h \psi_{g}(x)$, all $g \in G$. In this case we say $\phi$ is rigid.

One systematic treatment of the transitive case is [27]. Another aspect of this case is [126].

If $G$ acting on $M$ is semisimple, but neither compact, nor acting transitively, nor linearly, there seems to be essentially no literature, at least that I know of. On the other hand, it would seem worthwhile to make efforts in this direction. These efforts could produce unifying theorems, shed light on the above three special cases, or be useful in geometry or physics. One possibility might be to extend some of the results of Parts I and II. We limit ourselves to a few remarks.

In the first place, the evidence is that the richness of actions of a noncompact semisimple Lie group will lie somewhere between the abelian case (extremely rich, e.g., $G=R$ ) and the compact group case (few actions, i.e., $G$ acts rigidly as mentioned above). We will try to make this point clearer.

In the linear theory, or representation theory, the semisimple case is close to the case of compact groups in that representations (finite dimensional) are rigid. This contrasts to the abelian case where even one dimensional representations of $R$ (up to equivalence) are parameterized by $R$.

This motivated the speculation that if $\phi: G \rightarrow \operatorname{Diff}(M)$ is an action of semisimple $G$ with fixed point $x \in M$, the representation $g \rightarrow D \phi_{g}$ : $T_{x}(M) \rightarrow T_{x}(M)$ determines the orbit structure of $\phi$ in a neighborhood of $x$. R. Hermann [44] showed this to be true formally, while Guillemin and Sternberg [37] show that this is actually true in the case of an analytic (real) action. On the other hand Guillemin and Sternberg [37] give a counterexample in the $C^{\infty}$ case for $G=\operatorname{SL}(2, R)$. This situation, however, is still not yet well understood. 
One might ask whether any action of a semisimple $G$ on a compact manifold is rigid. This is false as the following simple example shows. Let $G=\mathrm{SL}(2, R)$ act on the unit tangent bundle $T$ of a 2 -manifold $M^{2}$ of genus greater than one by dividing out a uniform discrete subgroup $\Gamma$ from $G$. These actions correspond to different complex structures on $M^{2}$ and thus one gets a continuous family of such actions. One sees this by considering $M$ as the double coset space $\Gamma \backslash G / K$ where $G / K$ is the complex upper half plane. See also for example [126].

This does not exclude the possibility of a number of cases of noncompact semisimple $G$ acting rigidly on compact $M$. Here is one such case. $G=\mathrm{SL}(n+1, R)$ acts transitively on $P^{n}(R)$ using homogeneous coordinates and in fact $\mathrm{SL}(n+1, R)$ has no other homogeneous spaces of dimension less than $n+1$. Thus there is at most one action, transitive or otherwise, of $\mathrm{SL}(n+1, R)$ on connected $M$ if the dimension of $M$ is less than $N+1$, so of course this action is rigid and $M$ must be $P^{n}(R)$ (or a point!).

This suggests that semisimple $G$ acting on $M$ of much lower dimension might be fairly amenable to study. The situation is akin to the work of Hsiang and Hsiang on compact $G$ [48].

The work of Hermann [43] and others on the (equivariant) compactification of homogeneous spaces may be interpreted as studying the action of a semisimple $G$ in the neighborhood of certain noncompact orbits.

I have just received a manuscript [47] of W. Y. Hsiang which is related to the material of this section.

IV.3. Final miscellany. We end by making some final remarks on the action of a Lie group $G$. The notion of induced representation which has proved useful in the linear theory has an analogue in the general case which we describe now. This construction generalizes the suspended action of $\S I I .1$. Suppose then $H$ is a closed subgroup of a Lie group $G$ and $\phi: H \rightarrow \operatorname{Diff}(M)$ is an action of $H$. Define an action $\psi: H \rightarrow \operatorname{Diff}(M \times G)$ by $\psi_{h}(m, g)=\left(\phi_{h}(m), g h\right)$ and let $\pi: M \times G \rightarrow E$ be the projection onto the orbit space. One obtains the following diagram where $f$ is induced by $\pi_{G}$.

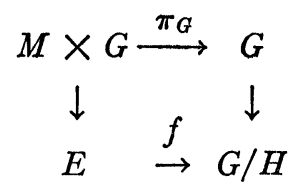

Here $E$ is a manifold and $f: E \rightarrow G / H$ is a bundle over $G / H$, Cartan's 
construction in [23]. The action $\sigma$ of $G$ on $M \times G$ defined by $\sigma_{g^{\prime}}(m, g)$ $=\left(m, g^{\prime} g\right)$ induces an action $\tau=\tau(\phi)$ of $G$ on $E$ which we might call the induced action of $\phi$. This action $\tau: G \rightarrow \operatorname{Diff}(E)$ is fiber preserving with respect to $f$ and commutes with translation on the base. $\tau$ restricted to $H$ leaves $f^{-1}(e H)$ invariant where it is equivalent to the original action $\phi$.

If $G=R, H=Z$, then $\tau$ is the construction of the suspension of a generator of $Z$ as in $\$ I I .1$. If $M$ is compact so is $E$ and if $\phi$ is linear, $M$ a vector space, then $E$ is a vector space bundle and the action of $G$ on sections is Mackey's induced representation.

We saw in Parts I and II that the concept of nonwandering points played a central role. A most important task would be to generalize this idea to a more general group $G$ and to formulate some of the conditions say of $\$ \S I .2$, I.3, I.6 for the case of a general Lie group, or even abelian, or semisimple $G$.

Palais in [79] considers a class of actions on noncompact groups which have many properties of compact transformation groups. These actions are quite restrictive in that the isotropy group is always compact and the manifold must be noncompact. These actions, however, resemble $G=Z$ acting on $M-\Omega$.

Suppose now that $\phi: G \rightarrow \operatorname{Diff}(M)$ is an action with a fixed point $x \in M$. Then the map $\tilde{\phi}: G \rightarrow \operatorname{Aut}\left(T_{x}(M)\right)$ is a linear representation of $G$, where $\tilde{\phi}(g)=D \phi_{g}(x)$ is the linear automorphism of $T_{x}\left(M_{i}\right)$ defined by the derivative of $\phi_{g}$ at $x$.

(3.1) Problem. To what extent (generically) does this representation determine the action of $G$ in a neighborhood of $x$, say up to conjugacy.

This is a basic local question. In earlier sections we saw aspects of it, starting with the stable manifold theorems, §I.2, in Sternberg [121] and Guillemin-Sternberg [37]. In general, the question is very far from being answered. Very likely, the higher derivatives will play a basic role for some groups.

Suppose more generally that $O=O_{x}$ is the compact orbit of some $x \in M$ of the action $\phi: G \rightarrow \operatorname{Diff}(M)$. Thus $G \rightarrow O_{x}, g \rightarrow \phi_{g}(x)$ is the orbit map with isotropy group $H_{x} \subset G$ acting on $M$ leaving $x$ fixed. The derivative $D \phi_{g}: T_{0}(M) \rightarrow T_{0}(M)$ defines a structure of a homogeneous vector space bundle (in the sense of [21]) on the restriction of the tangent bundle of $M$ restricted to $O$. We may generalize (3.1) with

(3.2) Problem. To what extent does the group of bundle automorphisms $D \phi_{g}: T_{0}(M) \rightarrow T_{0}(M)$ determine the action of $G$ in a neighborhood of $O$ ?

Here of course (3.1) is the case $O$ is a single point. For the notion 
of equivalence in (3.2), one might take orbit preserving homeomorphism. An earlier special case of (3.2) was discussed in $\$ I I .2$, where $G=R$ and $O$ was a closed orbit, i.e., the circle $S^{1}$.

For global actions of $G$ on $M$, it is probably profitable to consider initially very restricted cases, for example, actions on 2-manifolds. In this case, the possible orbits are well known, see Mostow [72]. Another tractable case might be actions with only two orbits.

\section{BIBLIOGRAPHY}

1. R. Abraham and J. Marsden, Foundations of mechanics, Benjamin, New York, 1967.

2. R. Abraham and J. Robbin, Transversal mappings and flows, Benjamin, New York, 1967.

3. R. L. Adler, A. G. Konheim and M. H. McAndrew, Topological entropy, Trans. Amer. Math. Soc. 114 (1965), 309--319.

4. R. L. Adler and M. H. McAndrew, The entropy of Chebyshev polynomials, Trans Amer. Math. Soc. 121 (1966), 236-241.

5. R. L. Adler and R. Palais, Homeomorphic-conjugacy of automorphisms on the torus, Proc. Amer. Math. Soc. 16 (1965), 1222-1225.

6. A. Andronov and L. Pontryagin, Systèmes grossiers, Dokl. Akad. Nauk. SSSR 14 (1937), 247-251.

7. D .V. Anosov, Roughness of geodesic flows on compact Riemannian manifolds of negative curvature, Soviet Math. Dokl. 3 (1962), 1068-1070.

8. - Ergodic properties of geodesic flows on closed Riemannian manifolds of negative curvature, Soviet Math. Dokl. 4 (1963), 1153-1156.

9. - Geodesic flows on compact Riemannian manifolds of negative curvature, Trudy Mat. Inst. Steklov. $90(1967)=$ Proc. Steklov Math. Inst. (to appear).

10. V. I. Arnold, Proof of a theorem of $A$. N. Kolmogorov on the invariance of quasiperiodic motions under small perturbations of the Hamiltonian, Russian Math. Surveys 18 (1963), 9-36.

11. V. I. Arnold and A. Avez, Problèmes ergodiques de la mécanique classique, Gauthier-Villars, Paris, 1966.

12. E. Artin and B. Mazur, On periodic points, Ann. of Math. (2) 81 (1965), 82-99.

12a. L. Auslander, L. Green, F. Hahn et al., Flows on homogeneous spaces, Princeton Univ. Press, Princeton, N. J., 1963.

13. A. Avez, Ergodic theory of dynamical systems, Vols. I, II, Univ. of Minneapolis, Minnesota, 1966, 1967.

14. P. Billingsley, Ergodic theory and information, Wiley, New York, 1965.

15. G. D. Birkhoff, Dynamical systems, Amer. Math. Soc. Colloq. Publ., vol. 9, Amer. Math Soc., Providence, R. I., 1927.

16. - Surface transformations and their dynamical applications, Acta Math. 43 (1920), 1-119.

17. - On the periodic motions of dynamical systems, Acta Math. So. (1927), 359-379. (See also Birkhoff's Collected Works.)

18. - Nouvelles recherches sur les systèmes dynamique, Mém. Pont. Acad. Sci. Novi Lyncaei 1 (1935), 85-216.

19. - Dynamical systems with two degrees of freedom, Trans. Amer. Math. Soc. 18 (1917), 199-300. 
20. A. Borel, et al., Seminar on transformation groups, Princeton Univ. Press, Princeton, N. J., 1960.

21. R. Bott, Homogeneous vector bundles, Ann. of Math. (2) 66 (1957), 203-248.

22. W. Boyce, Commuting functions with no common fixed point, Abstract 67T-218, Notices Amer. Math. Soc. 14 (1967), 280.

23. H. Cartan, Seminaire H. Cartan espaces fibrés et homotopie, Mimeographed, Paris, 1949-50.

24. E. Coddington and N. Levinson, Theory of ordinary differential equations, McGraw-Hill, New York, 1955.

25. J. Dieudonné, Foundations of modern analysis, Academic Press, New York, 1960.

26. A. Dold, Fixed point index and fixed point theorem for Euclidean neighborhood retracts, Topology 4 (1965), 1-8.

27. E. Dynkin, The maximal subgroups of the classical groups, Amer. Math. Soc. Transl. (2) 6 (1957), 245-379.

28. R. Ellis, Distal transformation groups, Pacific J. Math. 8 (1958), 401-405.

29. - The construction of minimal discrete flows, Amer. J. Math. 87 (1965), 564-574.

30. L. E. Elsgolts, An estimate for the number of singular points of a dynamical system defined on a manifold, Amer. Math. Soc. Transl. (1) 5 (1962), 498-510 (originally 68 (1952)).

31. F. B. Fuller, The existence of periodic points, Ann. of Math. (2) 57 (1953), 229230.

32. - - An index of fixed point type for periodic orbits, Amer. J. Math. 89 (1967), 133-148.

33. H. Furstenberg, The structure of distal flows, Amer. J. Math. 85 (1963), 477515.

34. I. M. Gelfand and S. V. Fomin, Geodesic flows on manifolds of constant negative curvature, Amer. Math. Soc. Transl. (2) 1 (1955), 49-66.

35. W. H. Gottschalk, Minimal sets: An introduction to topological dynamics, Bull. Amer. Math. Soc. 64 (1958), 336-351.

35a. W. H. Gottschalk and G. A. Hedlund, Topological dynamics, Amer. Math. Soc. Colloq. Publ., vol. 36, Amer. Math. Soc., Providence, R. I., 1955.

36. E. Grosswald, Topics from the theory of numbers, Macmillan, New York, 1966.

37. V. Guillemin and S. Sternberg, On a conjecture of Palais and Smale, Trans. Amer. Math. Soc. (to appear).

38. B. Halpern, Fixed points for iterates, (to appear).

39. P. Hartman, Ordinary differential equations, Wiley, New York, 1964.

40. J. Hadamard, Les surfaces à courbures opposées e leur lignes géodèsiques, J. Math. Pures Appl. 4 (1898), 27-73.

41. A. Haefliger, Variêtés feuilletées, Ann. Scuola Norm. Sup. Pisa (3) 16 (1962), 367-397.

42. G. Hedlund, The dynamics of geodesic flows, Bull. Amer. Math. Soc. 45 (1939), 241-246.

43. R. Hermann, Lie groups for physicists, Benjamin, New York, 1966.

44. - The formal linearization of a semisimple Lie algebra of vector fields about a singular point, Trans. Amer. Math. Soc. (to appear).

45. E. Hopf, Ergodic theory, Springer, Berlin, 1937.

46. - Statistik der geodätischen Linien in Mannigfaltigkeiten negativer Krümmung, Ber. Verh. Sächs. Akad. Wiss. Leipzig 91 (1939), 261-304.

47. W. Y. Hsiang, Remarks on differentiable actions of noncompact semi-simple Lie groups on Euclidean spaces, Amer. J. Math. (to appear). 
48. W. C. Hsiang, and W. Y. Hsiang, Differentiable actions of compact connected classical groups, Amer. J. Math. (to appear).

49. J. P. Huneke, Two counterexamples to a conjecture on commuting continuous functions of the closed unit interval, Abstract 67T-231, Notices Amer. Math. Soc. 14 (1967), 284.

50. G. Julia, Mémoire sur l'iteration des fonctions rationnelles, J. Math. Pures Appl. 4 (1918), 47-245.

51. S. Kobayashi and J. Eells, Problems in differential geometry, Proc. Japan-United States Seminar in Differential Geometry, Kyoto, Japan 1965, Nippon Hyoransha, Tokyo, 1966, pp. 167-177.

52. A. N. Kolmogoroff, On conservation of conditionally periodic motions for a small charge in Hamilton's function, Dokl. Akad. Nauk SSSR 98 (1954), 527-530. (Russian)

53. N. Kopell, Thesis, Univ. of California, Berkeley, 1967.

54. B. Kostant, Lie algebra cohomology and the generalized Borel-Weil theorem, Ann. of Math. (2) 74 (1961), 329-387.

55. I. Kupka, Contribution à la thêorie des champs gênériques, Contributions to Differential Equations 2 (1963), 457-484.

56. S. Lang, Introduction to differentiable manifolds, Wiley, New York, 1962.

57. S. Lefschetz, Differential equations: Geometric theory, Interscience, New York, 1957.

58. N. Levinson, $A$ second order differential equation with singular solutions, Ann. of Math. (2) 50 (1949), 127-153.

59. E. Lima, Commuting vector fields on $S^{3}$, Ann. of Math. (2) 81 (1965), 70-81.

60. - Common singularities of commuting vector fields on 2-manifolds, Comment. Math. Helv. 39 (1964), 97-110.

61. A. Malcev, On a class of homogeneous spaces, Amer. Math. Soc. Transl. (1) 9 (1962), 276-307 (originally 39 (1951)).

62. L. Markus, Structurally stable differential systems, Ann. of Math. (2) 73 (1961), $1-19$.

63. Y. Matsushima, On the discrete subgroups and homogeneous spaces of nilpotent Lie groups, Nagoya Math. J. 2 (1951), 95-110.

64. F. Mautner, Geodesic flows on symmetric Riemann spaces, Ann. of Math. (2) 65 (1957), 416-431.

65. K. Meyer, Energy functions for Morse-Smale systems, Amer. J. Math. (to appear).

66. D. Montgomery and L. Zippen, Topological transformation groups, Interscience, New York, 1955.

67. C. Moore, Distal affine transformation groups, Amer. J. Math (to appear).

68. M. Morse, The calculus of variations in the large, Amer. Math. Soc. Colloq. Publ., vol. 18, Amer. Math. Soc., Providence, R. I., 1934.

69. - $A$ one to one representation of geodesics on a surface of negative curvature, Amer. J. Math. 43 (1921), 33-51.

70. J. Moser, On invariant curves of area-preserving mappings of an annulus, Nachr. Akad. Wiss. Göttingen Math.-Phys. K1. II 1962, 1-20.

71. - Perturbation theory for almost periodic solutions for undamped nonlinear differential equations, Internat. Sympos. Nonlinear Differential Equations and Nonlinear Mechanics, Academic Press, New York, 1963, pp. 71-79.

72. G. D. Mostow, The extensibility of local Lie groups of transformations and groups on surfaces, Ann. of Math. (2) 52 (1950), 606-636.

73. J. Nash, Real algebraic manifolds, Ann. of Math. (2) 56 (1952), 405-421.

74. V. V. Nemytskii, Some modern problems in the qualitative theory of ordinary differential equations, Russian Math. Surveys 20 (1965), 1-35. 
75. K. Nomizu, On the cohomology of compact homogeneous spaces of nilpotent Lie groups, Ann. of Math. (2) 59 (1954), 531-538.

76. S. P. Novikov, The topology summer Institute, Seattle 1963, Russian Math. Surveys 20 (1965), 145-167.

77. S. P. Novikov, Smooth foliations on three-dimensional manifolds, Russian Math. Surveys 19 (1964) No. 6, 79-81.

78. J. C. Oxtoby and S. M. Ulam, Measure-preserving homeomorphisms and metrical transitivity, Ann. of Math. (2) 42 (1941), 874-920.

79. R. S. Palais, On the existence of slices for actions of non-compact Lie groups, Ann. of Math. (2) 73 (1961), 295-323.

80. - Equivalence of nearby differentiable actions of a compact group, Bull. Amer. Math. Soc. 67 (1961), 362-364.

81. R. S. Palais and T. Stewart, Deformations of compact differentiable transformation groups, Amer. J. Math. 82 (1960), 935-937.

82. J. Palis, Thesis, Univ. of California, Berkeley, 1967.

83. M. Peixoto, On structural stability, Ann. of Math. (2) 69 (1959), 199-222.

84. - Structural stability on two-dimensional manifolds, Topology 1 (1962), 101-120.

85. - Symposium on Differential Equations and Dynamical Systems (Puerto Rico) Academic Press, New York, 1967.

86. - On an approximation theorem of Kupka and Smale, J. Differential Equations 3 (1967), 214-227.

87. M. Peixoto and C. Pugh, Structurally stable systems on open manifolds are never dense, (to appear).

88. O. Perron, Die stabilitatsfrage bei differentialgleichungen, Math. Z. 32 (1930), 703-728.

89. H. Poincare, Sur les courbes dèfinies par des équations différentielles, J. Math. $4^{\circ}$ serie 1885 .

90. — Les méthodes nouvelles de la mécanique céleste, Vols. I, II, III, Paris, 1892, 1893, 1899; reprint, Dover, New York, 1957.

91. C. Pugh, The closing lemma, Amer. J. Math. (to appear)

92. —_, An improved closing lemma and a general density theorem, Amer. J. Math. (to appear).

93. - - The closing lemma for Hamiltonian systems, Proc. Internat. Congress Math. at Moscow, 1966.

94. G. Reeb, Sur certaines propriétés topologiques des trajectoires des systemes dynamiques, Acad. Roy. Belg. Cl. Sci. Mem. Coll. $8^{\circ} 27$ (1952), no. 9.

95. L. Reizinš, On systems of differential equations satisfying Smale's conditions, Latvijas PSR Zinātnu Akad. Vēstis Fiz. Tehn. Zinātnu Ser. 1964, 57-61. (Russian)

96. F. Riesz, and B. Nagy, Functional analysis, Ungar, New York, 1955.

97. $\mathrm{H}$. Rosenberg, A generalization of Morse-Smale inequalities, Bull. Amer. Math. Soc. 70 (1964), 422-427.

98. - Actions of $R^{n}$ on manifolds, Comment. Math. Helv. 41 (1966-67), 170-178. 102.

99. R. Sacksteder, Foliations and pseudo-groups, Amer. J. Math. 87 (1965), 79-

100. - Degeneracy of orbits of actions of $R^{n}$ on a manifold, Comment. Math.

Helv. 41 (1966-67), 1-9.

101. O. F. G. Shilling, Arithmetical algebraic geometry, Harper and Row, New York, 1956.

102. A. J. Schwartz, A generalization of a Poincare-Bendixon theorem to closed twodimensional manifolds, Amer. J. Math. 85 (1963), 453-458. 
103. - Common periodic points of commuting functions, Michigan Math. J. 12 (1965), 353-355.

104. A. Schwartz, Vector fields on a solid torus (to appear).

105. H. Seifert, Closed integral curves in 3-space and isotopic two-dimensional deformations, Proc. Amer. Math. Soc. 1 (1950), 287-302.

106. A. Selberg, Harmonic-analysis and discontinuous groups in weakly symmetric Riemann spaces with applications to Dirichlet series, J. Indian Math. Soc. 20 (1956), $47-87$.

107. L. P. Shil'nikov, The existence of an enumerable set of periodic motions in the neighborhood of a homoclinic curve, Dokl. Akad. Nauk SSSR 172 (1967), 298-301= Soviet Math. Dokl. 8 (1967), 102-106.

108. M. Shub, Thesis, Univ. of California, Berkeley, 1967.

109. S. Smale, Morse inequalities for a dynamical system, Bull. Amer. Math. Soc. 66 (1960), 43-49.

110. - On gradient dynamical systems, Ann. of Math. (2) 74 (1961), 199-206.

111. - On dynamical systems, Bol. Soc. Mat. Mexicana (2) 5 (1960), 195198.

112. - Dynamical systems and the topological conjugacy problem for diffeomorphisms, Proc. Internat. Congress Math. (Stockholm, 1962), Inst. Mittag-Leffler, Djursholm, 1963, pp. 490-496.

113. - A structurally stable differentiable homeomorphism with an infinite number of periodic points, Proc. Internat. Sympos. Non-linear Vibrations, vol. II, 1961, Izdat. Akad. Nauk Ukrain SSR, Kiev, 1963.

114. - Stable manifolds for differential equations and diffeomorphisms, Ann. Scuola Norm. Sup. Pisa (3) 17 (1963), 97-116.

115. - Diffeomorphisms with many periodic points, Differential and Combinatorial Topology, Princeton Univ. Press, Princeton, N. J., 1965, pp. 63-80.

116. - Structurally stable systems are not dense, Amer. J. Math. 88 (1966), $491-496$.

117. - Dynamical systems on n-dimensional manifolds, Symposium on differential equations and dynamical systems (Puerto Rico) Academic Press, New York, 1967.

118. J. Sotomayor, Generic one-parameter families of vector fields on two-dimensional manifolds (to appear).

119. P. R. Stein and S. M. Ulam, Non-linear transformations studies on electronic computers, Rozprawy Mat. 39 (1964).

120. S. Sternberg, Local contractions and a theorem of Poincaré, Amer. J. Math. 79 (1957), 787-789.

121. - On the structure of local homeomorphisms of Euclidean $n$-space. II, Amer. J. Math. 80 (1958), 623-631.

122. - The structure of local homeomorphisms. III, Amer. J. Math. 81 (1959), $578-604$.

123. —, Lectures on differential geometry, Prentice-Hall, Englewood Cliffs, N. J., 1964.

124. R. Thom, Sur une partition en cellules associes d unefunction sur une variete, C. R. Acad. Sci. Paris 228 (1949), 973-975.

125. A. Weil, Adeles and algebraic groups, Notes, Institute for Advanced Study, Princeton, N. J., 1961.

126. - On discrete subgroups of Lie groups. II, Ann. of Math. (2) 75 (1962), $578-602$.

127. R. Williams, One dimensional non-wandering sets (to appear). 